\title{
Extraction and validation of a new set of CMS PYTHIA8 tunes from underlying-event measurements
}

\author{
CMS Collaboration* \\ CERN, 1211 Geneva 23, Switzerland \\ Received: 28 March 2019 / Accepted: 20 November 2019 / Published online: 3 January 2020 \\ (C) CERN for the benefit of the CMS collaboration 2019
}

\begin{abstract}
New sets of CMS underlying-event parameters ("tunes") are presented for the PYTHIA8 event generator. These tunes use the NNPDF3.1 parton distribution functions (PDFs) at leading (LO), next-to-leading (NLO), or nextto-next-to-leading (NNLO) orders in perturbative quantum chromodynamics, and the strong coupling evolution at LO or NLO. Measurements of charged-particle multiplicity and transverse momentum densities at various hadron collision energies are fit simultaneously to determine the parameters of the tunes. Comparisons of the predictions of the new tunes are provided for observables sensitive to the event shapes at LEP, global underlying event, soft multiparton interactions, and double-parton scattering contributions. In addition, comparisons are made for observables measured in various specific processes, such as multijet, Drell-Yan, and top quarkantiquark pair production including jet substructure observables. The simulation of the underlying event provided by the new tunes is interfaced to a higher-order matrix-element calculation. For the first time, predictions from PYTHIA8 obtained with tunes based on NLO or NNLO PDFs are shown to reliably describe minimum-bias and underlying-event data with a similar level of agreement to predictions from tunes using LO PDF sets.
\end{abstract}

\section{Introduction}

Monte Carlo (MC) simulation codes describe hadron-hadron collisions with models based on several components. The hard scattering component of the event consists of particles from the hadronization of partons whose kinematics are predicted using perturbative matrix elements (MEs), along with partons from initial-state radiation (ISR) and final-state radiation (FSR) that are simulated using a showering algorithm. The underlying event (UE) consists of the beam-beam remnants (BBR) and the particles that arise from multiple-parton interactions (MPI). The BBR are what remains after a parton is scattered out of each of the two initial beam hadrons.

^ e-mail: cms-publication-committee-chair@cern.ch
The MPI are additional soft or semi-hard parton-parton scatterings that occur within the same hadron-hadron collision. Generally, observables sensitive to the UE also receive contributions from the hard-scattering components. Accurately describing observables that are sensitive to the UE not only requires a good description of BBR and MPI, but also a good modeling of hadronization, ISR, and FSR. Standard MC event generators, such as PYTHIA8 [1], HERWIG [2,3], and SHERPA [4] have adjustable parameters to control the behavior of their event modeling. A set of these parameters, which has been adjusted to better fit some aspects of the data, is referred to as a tune.

In a previous study [5], we presented several PYTHIA8 and HERWIG++ UE tunes constructed for a center-of-mass energy $\sqrt{s}$ lower than $13 \mathrm{TeV}$. The CMS PYTHIA8 tune CUETP8M1 is based on the Monash tune [6], both using the NNPDF2.3LO parton distribution function (PDF) set [7]. The CMS PYTHIA8 tune CUETP8S1-CTEQ6L1 is based instead on the tune $4 \mathrm{C}[8]$. Both tunes CUETP8M1 and CUETP8S1-CTEQ6L1 were constructed by fitting the CDF UE data at $\sqrt{s}=900 \mathrm{GeV}$ and $1.96 \mathrm{TeV}$ [9] together with CMS UE data at $\sqrt{s}=7 \mathrm{TeV}$ [10]. A similar procedure was used for the determination of the HERWIG++ tune (CUETHppS1) with the CTEQ6L1 PDF set [11]. A collection of previously published tunes is documented in $[6,8,12,12-15]$.

In this paper, a new set of tunes for the UE simulation in the PYTHIA8 (version 8.226) event generator is obtained by fitting various measurements sensitive to soft and semi-hard MPI at different hadron collision energies [9,10], including data from $\sqrt{s}=13 \mathrm{TeV}$ [16]. These tunes are constructed with the leading order (LO), next-to-leading order (NLO), and next-to-next-to-leading order (NNLO) versions of the NNPDF3.1 PDF set [17] for the simulation of all UE components. Typically, the values of strong coupling used for the simulation of the hard scattering are chosen consistent with the order of the PDF set used.

The new tunes are obtained by fitting CDF UE data at $\sqrt{s}=1.96 \mathrm{TeV}$ [9], together with CMS UE data at $\sqrt{s}=$ $7 \mathrm{TeV}[10]$ and at $13 \mathrm{TeV}[16,18]$. For the first time, we show 
that predictions obtained with tunes based on higher-order PDF sets are able to give a reliable description of minimumbias (MB) and UE measurements with a similar level of agreement to predictions from tunes using LO PDF sets. We also compare the predictions for multijet, Drell-Yan, and top-antiquark $(\mathrm{t} \overline{\mathrm{t}})$ processes from PYTHIA8 with new tunes in ME-parton shower (PS) merged configurations.

In Sect. 2 we describe observables that are sensitive to $\mathrm{MB}$ and UE: diffractive processes [19], where one or both protons remain intact after the collision; and double-parton scattering (DPS), where two hard scatterings occur within the same collision. In Sect. 3, we compare the tunes that were constructed before the data at $\sqrt{s}=13 \mathrm{TeV}$ were available ("Pre-13 TeV" tunes) with UE data measured at $13 \mathrm{TeV}$. Section 4 is dedicated to a general discussion of the choice of PDF sets and strong coupling values for the UE simulation. In Sect. 5 we describe the new tunes. Section 6 shows the validation of the new CMS PYTHIA8 tunes for multijet, Drell-Yan, $\bar{t} \bar{t}$, and DPS processes. Section 7 is the summary and conclusions.

\section{Observables for characterizing minimum bias, underlying event, and double-parton scattering}

Minimum bias is a generic term that refers to inelastic events that are collected with a loose event selection that has the smallest bias possible. The MB observables are constructed from data with little or no additional selection requirements. The majority of MB collisions are soft, with a typical transverse momentum scale $p_{\mathrm{T}} \lesssim 2 \mathrm{GeV}$. The UE is defined as the activity that is not associated with the particles originating from the hard scattering of two partons and is generally studied in events that contain a hard scattering with $p_{\mathrm{T}} \gtrsim 2 \mathrm{GeV}$. The main contribution to the UE comes from color exchanges between the beam partons and is modeled in terms of MPI, $\mathrm{BBR}$, and color reconnection (CR). The MB and UE observables have quite different kinematic properties because they are affected by different mixtures of hard and soft scattering processes.

As illustrated in Fig. 1, one can use the topological structure of a typical hard hadron-hadron collision to study the UE experimentally. On an event-by-event basis, a leading object is used to define regions of $\eta-\phi$ space that are sensitive to the modeling of the UE, where $\eta$ is the pseudorapidity and $\phi$ is the azimuthal scattering angle defined in the $x y$ plane. The azimuthal separation between charged particles and the leading object, $\Delta \phi=\phi-\phi_{\max }$, is used to define the UE-sensitive regions. Here $\phi_{\max }$ is the azimuth of the leading object and $\phi$ is the azimuth angle of an outgoing charged particle. The regions are labelled as 'toward' $\left(|\Delta \phi| \leq 60^{\circ}\right)$, 'away' $\left(|\Delta \phi|>120^{\circ}\right)$, and 'transverse' $\left(60^{\circ}<|\Delta \phi| \leq 120^{\circ}\right)$. The transverse region can further

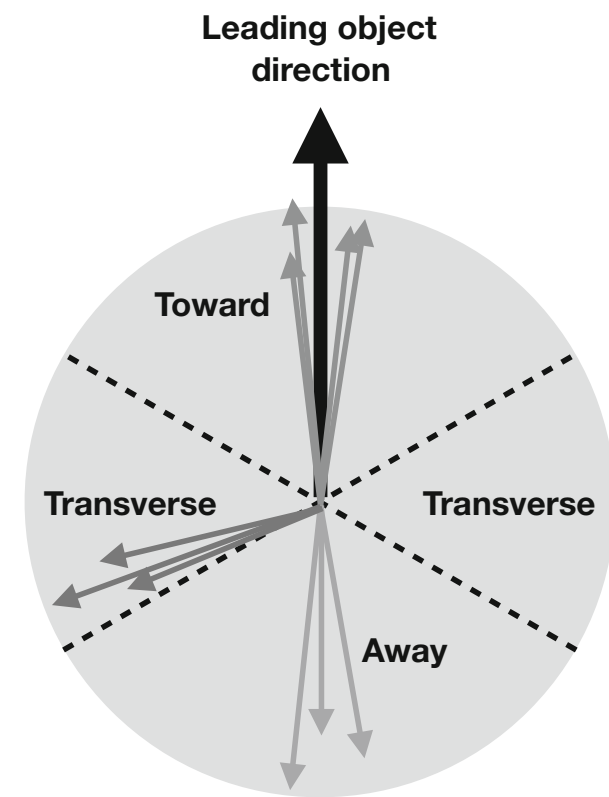

Fig. 1 Illustration of several $\phi$ regions relative to the leading object that are sensitive to the underlying event. See the text for the details on the definitions of the regions

be separated into transMAX and transMIN. On an event-byevent basis, transMAX (transMIN) is defined as the transverse region having the maximum (minimum) of either the number of charged particles, or scalar $p_{\mathrm{T}}$ sum of charged particles $\left(p_{\mathrm{T}}^{\text {sum }}\right)$, depending on the quantity under study.

Published UE studies used the charged-particle jet with the largest $p_{\mathrm{T}}$ [16], the dilepton system in DY [20,21], or $\mathrm{t} \overline{\mathrm{t}}$ [22] events as the leading (i.e., the highest $p_{\mathrm{T}}$ ) objects. The tunes from CDF and CMS data $[9,10]$ made use of the charged particle with the largest $p_{\mathrm{T}}\left(p_{\mathrm{T}}^{\max }\right)$ as the "leading object", and use only charged particles with $p_{\mathrm{T}}>0.5 \mathrm{GeV}$ and $|\eta|<0.8$ to characterize the UE. The toward region contains the leading object, and the away region is expected to include the object recoiling against the leading one. Most of the UE contributions, i.e., PS and MPI, are contained in the two transverse regions. For events with multiple ISR or FSR emissions, transMAX often contains a third hard jet, while both transMAX and transMIN receive contributions from the MPI and BBR components. Typically, the transMIN observables are more sensitive to the MPI and BBR components of the UE.

Observables sensitive to UE contributions are the chargedparticle multiplicity and the charged-particle scalar- $p_{\mathrm{T}}$ sum densities in the $\eta-\phi$ space, measured in transMIN and transMAX. The tunes that are constructed by fitting such UEsensitive observables are referred to as "UE tunes".

The PYTHIA8 MC event generator also simulates singlediffractive (SD) dissociation, double-diffractive (DD) dissociation, central-diffractive (CD), and nondiffractive (ND) processes [23], which contribute to the inelastic cross section 
in hadron-hadron collisions. In SD, CD, and DD events, one or both of the beam particles are excited into color singlet states, which then decay. The SD and DD processes correspond to color singlet exchanges between the beam hadrons, while $\mathrm{CD}$ corresponds to double color singlet exchange with a diffractive system produced centrally. For ND processes, color exchanges occur, the outgoing remnants are no longer color singlets, and a multitude of particles is produced. All processes except SD are defined as nonSD (NSD) processes. An NSD-enhanced sample is required to have an energy deposit in both the backward $(-5<\eta<-3)$ and the forward $(3<\eta<5)$ regions of the detector. The details of the selection for different types of diffractive events can be found in Ref. [24].

Generally, MC models such as PYTHIA8 regularize the contributions of the primary hard-scattering processes and MPI to the differential cross section by using a threshold parameter $p_{\mathrm{T}}^{0}$. The primary hard-scattering processes and the MPI are regularized in the same way with this parameter. This threshold is expected to have a dependence on the center-ofmass energy of the hadron-hadron collision, $\sqrt{s}$. The threshold at a reference center-of-mass energy $\sqrt{s}=7 \mathrm{TeV}$ is called pT0Ref. In PYTHIA8 the energy dependence is parameterized using a power law function with a reference energy parameter $s_{0}$ and an exponent $\epsilon$. At a given center-of-mass energy, the amount of MPI depends on the threshold $p_{\mathrm{T}}^{0}$, the PDF, and the overlap of the matter distributions of the two colliding hadrons. Smaller values of $p_{\mathrm{T}}^{0}$ result in larger MPI contributions because of a higher MPI cross section. Each MPI adds colored partons to the final state, creating a dense net of color lines that spatially overlap with the fields produced by the partons of the hard scattering and with each other. All the generated color lines may connect to each other according to the CR model.

Since PYTHIA8 regularizes both the cross section for MPI and the cross section of collisions with low- $p_{\mathrm{T}}$ exchange using the $p_{\mathrm{T}}^{0}$ parameter, one can model the overall ND cross section by letting the $p_{\mathrm{T}}$ of the primary hard scattering become small. In this simple approach, the UE in a hard-scattering process is related to MB collisions. At the same center-of-mass energy, the activity in the UE of a hardscattering process is greater than that of an average MB collision. In PYTHIA8, this is caused by the higher MPI activity in hard-scattering processes compared to a typical MB collision. By demanding a hard scattering, one forces the collision to be more central, i.e., with a small impact parameter between the protons, and this increases the probability of MPI. For MB collisions, peripheral collisions, where the impact parameter between the two colliding protons is large, are most common.

Typically MPI interactions contain particles with substantially lower $p_{\mathrm{T}}$ ("softer"). However, occasionally two hard 2-to-2 parton scatterings can occur within the same hadron- hadron collision. This is referred to as DPS. Tunes that are constructed by fitting DPS-sensitive observables are referred to as "DPS tunes". Ultimately, one universal tune that simultaneously accurately describes observables in hard scattering events, as well as MB collisions, is desirable.

The goals of this paper are to produce improved $13 \mathrm{TeV}$ PYTHIA8 tunes with well-motivated parameters, and to provide an investigation of the possible choices that can be made in PYTHIA8 which simultaneously describe a wide range of $\mathrm{UE}$ and $\mathrm{MB}$ measurements and are suitable for merged configurations, where a ME calculation is interfaced to the simulation of UE contributions.

\section{Comparisons of predictions for UE observables from previous tunes to measurements at $13 \mathrm{TeV}$}

In this section, comparisons are presented between data collected at $\sqrt{s}=13 \mathrm{TeV}$ and predictions from tunes obtained using fits to measurements performed at lower center-ofmass energies. Figure 2 displays comparisons of CMS data at $13 \mathrm{TeV}$ [16] for the transMIN and transMAX chargedparticle $p_{\mathrm{T}}^{\text {sum }}$ densities, as functions of the leading chargedparticle $p_{\mathrm{T}}^{\mathrm{max}}$. The data are compared with predictions from the PYTHIA8 tunes CUETP8S1-CTEQ6L1 [5], CUETP8M1 [5], and Monash [6].

The CMS Monash-based tune CUETP8M1 does not describe the central values of the data at $\sqrt{s}=13 \mathrm{TeV}$ well, nor does the original Monash tune. For example, CUETP8M1 and Monash tunes do not predict enough UE activity in the region with $p_{\mathrm{T}}^{\max }>5 \mathrm{GeV}$ (the "plateau" region) of transMIN at $13 \mathrm{TeV}$, with a disagreement of $\approx 10 \%$ and $\approx 5 \%$, respectively. The transMIN observables are very sensitive to MPI, which suggests that tune CUETP8M1 does not produce enough charged particles at $13 \mathrm{TeV}$. In addition, CUETP8M1 does not provide a good fit to the jet multiplicity in t $\bar{t}$ production either at $8 \mathrm{TeV}$ or at $13 \mathrm{TeV}[25,26]$. High jet multiplicity $t \bar{t}$ events are sensitive to the modeling of the ISR. Hence, CUETP8M1 may not have the proper mixture of MPI and ISR. The ATLAS collaboration has also observed some discrepancies between the predictions of the A14 tune [12], used as standard tune for analyses of 7 and $8 \mathrm{TeV}$ data, and the data at $13 \mathrm{TeV}$ [27].

The CMS UE tunes were constructed by fitting CDF UE data at $\sqrt{s}=900 \mathrm{GeV}$ and $1.96 \mathrm{TeV}$, together with CMS UE data at $\sqrt{s}=7 \mathrm{TeV}$. In Fig. 2 the CMS UE tunes provide a fairly good description of the $13 \mathrm{TeV}$ UE data. Because the CMS UE tunes were obtained by fitting UE observables at various collision energies $(\sqrt{s}=900,1960$, and $7000 \mathrm{GeV})$, they underestimate the data at $\sqrt{s}=13 \mathrm{TeV}$. This might be an indication of the need to improve the energy extrapolation function implemented in PYTHIA8 [28]. Predictions obtained with the Monash tune, which is the default PYTHIA8 tune, 

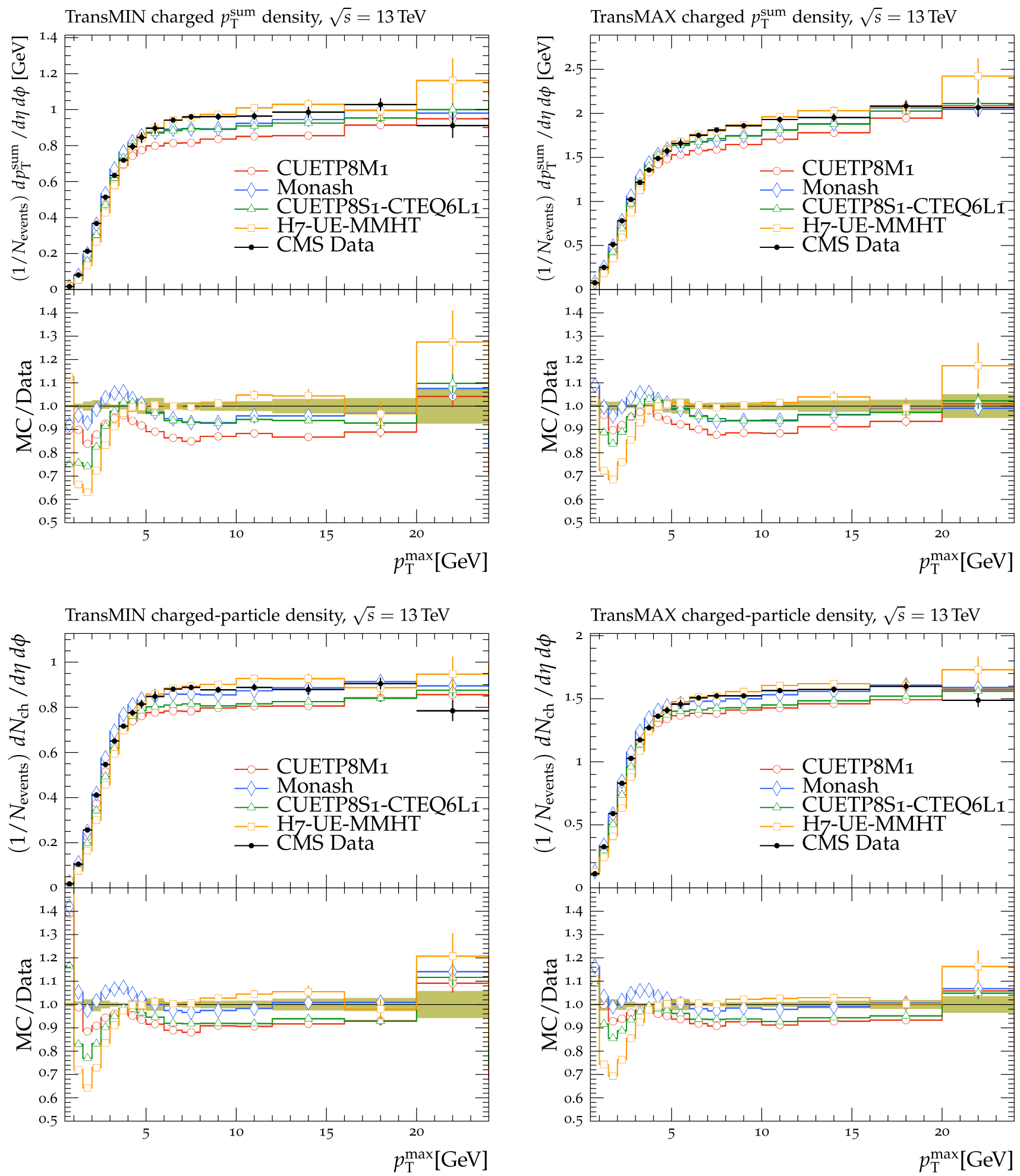

Fig. 2 The (left column) transMIN and (right column) transMAX charged-particle $p_{\mathrm{T}}^{\text {sum }}$ (upper row), and multiplicity (lower row) densities for particles with $p_{\mathrm{T}}>0.5 \mathrm{GeV}$ in $|\eta|<2.0$, as a function of the transverse momentum of the leading charged particle $\left(p_{\mathrm{T}}^{\max }\right)$, from the CMS $\sqrt{s}=13 \mathrm{TeV}$ analysis [16]. The data are compared with the PYTHIA8 tune Monash, the CMS PYTHIA8 tunes CUETP8S1-CTEQ6L1 and CUETP8M1, and the HERWIG7 (labelled as "H7") tune UE-MMHT.

The ratios of the simulations to the data (MC/Data) are also shown, where the shaded band indicates the total experimental uncertainty in the data. Vertical lines drawn on the data points refer to the total uncertainty in the data. Vertical lines drawn on the MC points refer to the statistical uncertainty in the predictions. Horizontal bars indicate the associated bin width 
slightly better reproduce the $13 \mathrm{TeV}$ UE data, but is somewhat worse at describing the UE observables at $\sqrt{s}=900$ and $1960 \mathrm{GeV}$ than the CMS UE tunes.

Predictions from the HERWIG7.1 tune UE-MMHT [3] are also shown. The H7-UE-MMHT tune was obtained by fitting UE data at $\sqrt{s}=0.9$ and $7 \mathrm{TeV}$. This tune is based on the MMHT2014 PDF set [29] and is able to describe the plateau region of the UE observables at $\sqrt{s}=13 \mathrm{TeV}$. The part of the spectrum at $p_{\mathrm{T}}^{\max }>5 \mathrm{GeV}$ is not well reproduced in the range of the leading charged-particle $p_{\mathrm{T}}^{\max }$ between 2 and $7 \mathrm{GeV}$, with differences of up to $30 \%$ with respect to the data. The predictions from HERWIG7 achieve an overall good agreement with measurements at $\sqrt{s}=7 \mathrm{TeV}$ [30], while the disagreement observed for measurements at $\sqrt{s}=$ $13 \mathrm{TeV}$ might indicate the need for further tuning of the new soft MPI model [30]. Since many parameters related to PS changed between HERWIG++ and HERWIG7, the CMS tunes extracted for HERWIG++ with the CTEQ6L1 PDF set and documented in Ref. [5] are not updated and should not be used with HERWIG7.

Since no currently available tune is able to optimally reproduce the UE data at $\sqrt{s}=13 \mathrm{TeV}$, we aim to produce improved PYTHIA8 UE tunes.

\section{PDF and strong coupling values for the tunes}

Two of the basic input parameters to the predictions are the choice of the order of the PDF sets and values of the strong coupling $\alpha_{S}$. These appear in the hard partonic MEs, the PS model, and the MPI model. The $\alpha_{S}$ values used in simulations at LO or NLO are typically different. Traditionally, the perturbative order of the PDF is matched to the order of the ME calculation. Merged calculations capture some higherorder corrections with respect to the formal order of the ME calculation. Merging schemes, such as the $k_{\mathrm{T}}-\mathrm{MLM}$ [31] or CKKW [32,33], allow the combination of predictions of jet production using ME calculations with those from PS emissions for soft and collinear parton radiation at leading-log accuracy without double counting or dead regions. Merging can be applied also for processes generated at NLO. Using the same PDF set and $\alpha_{S}$ value in the ME calculations and in the simulation of the various components of the PS is advocated in Ref. [34], and by the HERWIG7 and SHERPA Collaborations, especially when the PS simulation is merged with calculations of higher-order MEs. The PDF used for the hard process is constrained by the accuracy of the ME calculation. If we require the PDF to match between the ME and PS, simulations with a (N)NLO ME will also require a (N)NLO PDF in the PS. Depending on the process, this may not have a significant effect. For PS MC event generators, dif- ferent strategies are adopted; CMS [5] and ATLAS [12] tunes are traditionally based on LO PDFs, PYTHIA8 [6] tunes are mostly based on LO PDFs, new SHERPA [4] tunes are based on NNLO PDFs, and HERWIG7 [30] provide tunes based on NLO PDFs. The usage of a LO PDF set in the UE simulation is motivated by the fact that MPI processes occur at very low energy scales, where a physical (positive) gluon distribution is required by the parton shower. However, there is no consensus on the choice of the order of the PDF. For example, in the NNPDF3.1 set at NNLO, the gluon distribution remains physical even at very low scales.

In the PYTHIA8 tunes produced prior to this paper, the values used for $\alpha_{S}$ were often not the same as those used in the PDFs. For example, in the Monash tune, the FSR $\alpha_{S}\left(m_{\mathrm{Z}}\right)$, set to 0.1365 , is obtained by fitting PYTHIA8 predictions to LEP event-shape measurements [6], the ISR $\alpha_{S}\left(m_{\mathrm{Z}}\right)$ is assumed to be equal to FSR $\alpha_{S}\left(m_{\mathrm{Z}}\right)$, and the hard scattering and MPI $\alpha_{S}\left(m_{\mathrm{Z}}\right)$ is set to 0.13 according to the value used in the LO PDF set. Even though the $\alpha_{S}$ values are free parameters in event generators and various possibilities are viable, the usual course is to choose them consistent with the value used by the PDF set.

In this paper, a collection of new tunes is presented for PDF sets that are evaluated at different accuracies and tested against observables of MB, UE, and hard processes. The NNPDF3.1 PDF sets at the LO, NLO, and NNLO accuracy are used [17]. The LO PDF set uses an $\alpha_{S}\left(m_{\mathrm{Z}}\right)$ value of 0.13 , while 0.118 [35] is the $\alpha_{S}\left(m_{\mathrm{Z}}\right)$ value used for the NLO and NNLO PDF sets. None of the central values of the PDF sets have negative values for any parton flavor in the phase space relevant for comparisons. Special care is required when applying these tunes at high- $\mathrm{x}$ regions, where the parton distributions in NNPDF3.1 NLO and NNLO PDF may become negative, which implies an unphysical (negative) value of the calculated cross sections.

The UE simulation is performed by PYTHIA8, together with PS merged with a calculation of a higher-order or a multileg ME provided by external programs, such as POWHEG [36] or MADGRAPH5_aMC@NLO (MG5_aMC) [37]. The issue of combining external ME calculations with PS contributions is addressed by the merging procedure. The procedures considered in this paper are the "FxFx" [38] or the "POWHEG" [39] methods for merging higher-order (NLO) MEs to PS and the "MLM" method [31].

During this study, we also investigated the effect of imposing an additional rapidity $(y)$ ordering to ISR in these merging calculations. The PYTHIA8 Monash tune includes a rapidity ordering for both ISR and MPI. The rapidity ordering acts as an extra constraint on the $p_{\mathrm{T}}$-ordered emissions, thus reducing the phase space for parton emission. 


\section{New CMS PYTHIA8 tunes at $13 \mathrm{TeV}$}

In the following, a set of new $13 \mathrm{TeV}$ PYTHIA8.226 tunes is presented with different choices of values of the strong coupling used in the modeling of the ISR, FSR, hard scattering, and MPI, as well as the order of its evolution as a function of the four-momentum squared $Q^{2}$. We distinguish the new tunes according to the order of the PDF set used: LO-PDF, NLO-PDF, or NNLO-PDF. The tunes are labeled as $\mathrm{CP} i$, where CP stands for "CMS PYTHIA8" and $\mathrm{i}$ is a progressive number from 1 to 5 . Only five parameters related to the simulation of MPI, to the overlap matter distribution function [40], and to the amount of CR are constrained for the new CMS tunes. In all tunes, we use the MPI-based CR model [41]. The CP tunes are multipurpose tunes, aiming for a consistent description of UE and MB observables at several collision energies and a reliable prediction of the UE simulation in various processes when merged with higher-order ME calculations.

The settings, used in the determination of the new CMS PYTHIA8 UE tunes, are as follows:

- Tune CP1 uses the NNPDF3.1 PDF set at LO, with $\alpha_{S}$ values used for the simulation of MPI, hard scattering, FSR, and ISR equal to, respectively, 0.13, 0.13, 0.1365, and 0.1365 , and running according to an LO evolution.

- Tune CP2 is a slight variation with respect to CP1, uses the NNPDF3.1 PDF set at LO, with $\alpha_{S}$ values used for the simulation of MPI, hard scattering, FSR, and ISR contributions equal to 0.13 , and running according to an LO evolution.

- Tune CP3 uses the NNPDF3.1 PDF set at NLO, with $\alpha_{S}$ values used for the simulation of MPI, hard scattering, FSR, and ISR contributions equal to 0.118 , and running according to an NLO evolution.

- Tune CP4 uses the NNPDF3.1 PDF set at NNLO, with $\alpha_{S}$ values used for the simulation of MPI, hard scattering, FSR, and ISR contributions equal to 0.118 , and running according to an NLO evolution.

- Tune CP5 has the same settings as CP4, but with the ISR emissions ordered according to rapidity.
The parameters related to the simulation of the hadronization and beam remnants are not varied in the fits and are kept fixed to the values of the Monash tune. The overlap distribution between the two colliding protons is modeled according to a double-Gaussian functional form with the parameters coreRadius and corefraction. This parametrization of the transverse partonic overlap of two protons identifies an inner, denser part, the so-called core, and an outer less dense part. The coreRadius parameter represents the width of the core and the corefraction, the fraction of quark and gluon content enclosed in the core. A double-Gaussian function is preferred for modeling the proton overlap over the negative exponential used in some previous tunes. Tunes using a double-Gaussian function tend to better reproduce the cross sections measured by the CMS experiment at $\sqrt{s}=7 \mathrm{TeV}$ [10], simultaneously as a function of charged-particle multiplicity and transverse momenta.

The parameter that determines the amount of simulated CR in the MPI-based model is varied in the fits. A small (large) value of the final-state CR parameter tends to increase (reduce) the final particle multiplicities.

The new CMS PYTHIA8 tunes are extracted by varying the parameters listed in Table 1 and by fitting UE observables at various collision energies. In the fitting procedure, we use the charged-particle and $p_{\mathrm{T}}^{\text {sum }}$ densities, measured in transMIN and transMAX regions as a function of $p_{\mathrm{T}}^{\max }$, as well as the charged-particle multiplicity as a function of pseudorapidity $\eta$, measured by CMS at $\sqrt{s}=13 \mathrm{TeV}[16,18]$. In addition, we also use the charged-particle and $p_{\mathrm{T}}^{\text {sum }}$ densities as a function of the leading charged-particle $p_{\mathrm{T}}$, measured in transMIN and transMAX by CMS at $\sqrt{s}=7 \mathrm{TeV}$ [10] and by $\mathrm{CDF}$ at $\sqrt{s}=1.96 \mathrm{TeV}[9]$.

Tunes are determined by generating sets of predictions using the RIVET [42] (version 2.5.2) and the PROFESSOR [43] (version 1.4.0) frameworks with around 150 different choices of the five parameter values used in the event simulation. The predictions form a grid in the five-dimensional parameter space which is fitted using a third-order polynomial function. The uncertainty introduced in the fitted parameters due to the interpolation procedure is negligible compared with the quoted tune uncertainty. Results are found to be stable if one decreases this number to 100 or increases to 200 , or uses a

Table 1 Parameters in the PYTHIA8 MC event generator together with the PDFs determine the energy dependence of MPI, the overlap matter distribution function, and the amount of simulated color reconnection. The parameter ranges used for the fits are also listed

\begin{tabular}{lll}
\hline Parameter description & Name in PYTHIA & Range considered \\
\hline MPI threshold (GeV), pTORef, at $\sqrt{s}=\sqrt{s_{0}}$ & MultipartonInteractions : pT0Ref & $1.0-3.0$ \\
Exponent of $\sqrt{s}$ dependence, $\epsilon$ & MultipartonInteractions : ecmPow & $0.0-0.3$ \\
Matter fraction contained in the core & MultipartonInteractions: coreFraction & $0.1-0.95$ \\
Radius of the core & MultipartonInteractions: coreRadius & $0.1-0.8$ \\
Range of color reconnection probability & ColorReconnection:range & $1.0-9.0$ \\
\hline
\end{tabular}


fourth-order polynomial function for the grid interpolation. The generated inelastic events include ND and diffractive $(\mathrm{DD}+\mathrm{SD}+\mathrm{CD})$ contributions. The UE observables used to determine the tunes are sensitive to diffractive contributions only at very small $p_{\mathrm{T}}^{\max }$ values $(<3 \mathrm{GeV})$. The ND component is dominant for $p_{\mathrm{T}}^{\max }$ values greater than $\approx 3.0 \mathrm{GeV}$, since the cross section of the diffractive components rapidly decreases as a function of the exchanged $p_{\mathrm{T}}$. Minimum-bias observables, such as the inclusive charged-particle multiplicity as a function of $\eta$, are sensitive to all contributions over the whole spectrum.

The fit is performed by minimizing the $\chi^{2}$ function

$\chi^{2}(p)=\sum_{\mathrm{O}_{j}} \sum_{i} \frac{\left(f_{i, \mathrm{O}_{j}}(p)-R_{i, \mathrm{O}_{j}}\right)^{2}}{\Delta_{i, \mathrm{O}_{j}}^{2}}$

where the sum runs over each bin $i$ of every observable $\mathrm{O}_{\mathrm{j}}$. The $f_{\mathrm{i}}(p)$ functions represent a parametrization of the dependence of the predictions in bin $i$ on the tuning parameters, $R_{\mathrm{i}}$ is the value of the measured observable in bin $i$, and $\Delta_{\mathrm{i}}$ is the total experimental uncertainty of $R_{\mathrm{i}}$. The best fit values of the tuned parameters are shown in Table 2 for CP1 and CP2, i.e., the tunes using LO PDF sets, and in Table 3 for CP3, $\mathrm{CP} 4, \mathrm{CP} 5$, i.e., the tunes using NLO or NNLO PDF sets. Uncertainties in the parameters of these tunes are discussed in Appendix A. No correlation across bins is included in the minimized $\chi^{2}$ function.

The value of pTORef and its energy dependence is very different between tunes based on LO PDF sets and tunes based on NLO or NNLO PDFs. While pTORef is around 2.3-2.4 GeV for $\mathrm{CP} 1$ and $\mathrm{CP} 2$ tunes with $\epsilon \approx 0.14-0.15$, $\mathrm{CP} 3, \mathrm{CP} 4$, and $\mathrm{CP} 5$ tunes prefer much lower values for both pTORef $(\approx 1.4-1.5)$ and $\epsilon(\approx 0.03-0.04)$. A value of $\epsilon$ of $\approx 0.03-0.04$ corresponds to a very weak energy dependence of the threshold of the MPI cross section. These results can be understood by considering the shapes of the gluon densities at small $x$ for the different PDF sets. In order to describe the UE observables, the rapidly increasing gluon densities at small $x$ in LO PDF sets favor large values of pTORef. Meanwhile NLO and NNLO PDF sets, whose gluon densities are more flat at low $x$, need higher contributions of MPI, i.e., a small value of pTORef. Figure 3 shows the number of MPI observed for the various tunes and the gluon distribution at a reference scale of $\mu=3 \mathrm{GeV}$ for various NNPDF versions. The larger number of simulated MPI for NLO and NNLO tunes with respect to LO tunes is apparent.

We have found that the values of pTORef and $\epsilon$ also depend on the order of the running used for $\alpha_{S}$. In particular, fits based on NLO or NNLO PDF sets, i.e., CP3, CP4, or CP5, with an $\mathrm{LO} \alpha_{S}$ running prefer even smaller values for both pTORef and $\epsilon$ than the ones in the tunes obtained with an NLO $\alpha_{S}$ running. This is because $\alpha_{S}$ runs faster at NLO than at $\mathrm{LO}$. When $\alpha_{S}$ is run from the same value at the same scale $\left(m_{\mathrm{Z}}\right)$, the effective coupling at low scales is larger for NLO running than for LO running. Therefore, a lower pTORef is needed for $\mathrm{NLO} \alpha_{S}$ running than for $\mathrm{LO} \alpha_{S}$ running to obtain a similar number of MPI.

For tunes based on NLO and NNLO PDF sets, the value of pTORef is as low as the initial scale of the PDF $Q_{\min }^{2}$. For interactions occurring at $Q^{2}$ which are lower than $Q_{\min }^{2}$, the value of the PDF is left frozen to the value assumed at the initial scale.

The contribution from CR also changes among the different tunes and depends on the choice of PDF and its order. In particular, the amount of CR is also affected by the shape of the PDFs at small fractional momenta $x$.

Parameters related to the overlap matter distribution function differ between the different tunes. They are strongly cor-
Table 2 CMS PYTHIA8 LO-PDF tunes CP1 and CP2. Both the values at $Q=m_{\mathrm{Z}}$ and the order of running with $Q^{2}$ of the strong coupling $\alpha_{S}\left(m_{\mathrm{Z}}\right)$ are listed. In these tunes, we use the Schuler-Sjöstrand diffraction model [44] and also include the simulation of $\mathrm{CD}$ processes. The number of degrees of freedom for tunes CP1 and CP2 is 63

\begin{tabular}{lll}
\hline PYTHIA8 parameter & CP1 & CP2 \\
\hline PDF Set & NNPDF3.1 LO & NNPDF3.1 LO \\
$\alpha_{S}\left(m_{\mathrm{Z}}\right)$ & 0.130 & 0.130 \\
SpaceShower:rapidityOrder & Off & Off \\
MultipartonInteractions : EcmRef $(\mathrm{GeV})$ & 7000 & 7000 \\
$\alpha_{S}^{\text {ISR }}\left(m_{\mathrm{Z}}\right)$ value/order & $0.1365 / \mathrm{LO}$ & $0.130 / \mathrm{LO}$ \\
$\alpha_{S}^{\mathrm{FSR}}\left(m_{\mathrm{Z}}\right)$ value/order & $0.1365 / \mathrm{LO}$ & $0.130 / \mathrm{LO}$ \\
$\alpha_{S}^{\mathrm{MPI}}\left(m_{\mathrm{Z}}\right)$ value/order & $0.130 / \mathrm{LO}$ & $0.130 / \mathrm{LO}$ \\
$\alpha_{S}^{\mathrm{ME}}\left(m_{\mathrm{Z}}\right)$ value/order & $0.130 / \mathrm{LO}$ & $0.130 / \mathrm{LO}$ \\
MultipartonInteractions : pT0Ref $(\mathrm{GeV})$ & 2.4 & 2.3 \\
MultipartonInteractions : ecmPow & 0.15 & 0.14 \\
MultipartonInteractions : coreRadius & 0.54 & 0.38 \\
MultipartonInteractions : coreFraction & 0.68 & 0.33 \\
ColorReconnection:range & 2.63 & 2.32 \\
$\chi^{2} /$ dof & 0.89 & 0.54
\end{tabular}


Table 3 CMS PYTHIA8 NLO-PDF tune CP3 and NNLO-PDF tunes CP4 and CP5. Both the values at $Q=m_{\mathrm{Z}}$ and the order of running with $Q^{2}$ of the strong coupling $\alpha_{S}$ are listed. In these tunes, we use the
Schuler-Sjöstrand diffraction model [44] and also include the simulation of $\mathrm{CD}$ processes. The number of degrees of freedom for tunes CP3, $\mathrm{CP} 4$, and $\mathrm{CP} 5$ is 63

\begin{tabular}{llll}
\hline PYTHIA8 parameter & CP3 & CP4 & CP5 \\
\hline PDF set & NNPDF3.1 NLO & NNPDF3.1 NNLO & 0.118 \\
$\alpha_{S}\left(m_{Z}\right)$ & 0.118 & Off & On \\
SpaceShower: rapidityOrder & Off & 7000 & 7000 \\
MultipartonInteractions : EcmRef $(\mathrm{GeV})$ & 7000 & $0.118 / \mathrm{NLO}$ & $0.118 / \mathrm{NLO}$ \\
$\alpha_{S}^{\text {ISR }}\left(m_{\mathrm{Z}}\right)$ value/order & $0.118 / \mathrm{NLO}$ & $0.118 / \mathrm{NLO}$ & $0.118 / \mathrm{NLO}$ \\
$\alpha_{S}^{\text {FSR }}\left(m_{\mathrm{Z}}\right)$ value/order & $0.118 / \mathrm{NLO}$ & $0.118 / \mathrm{NLO}$ & $0.118 / \mathrm{NLO}$ \\
$\alpha_{S}^{\text {MPI }}\left(m_{\mathrm{Z}}\right)$ value/order & $0.118 / \mathrm{NLO}$ & $0.118 / \mathrm{NLO}$ & $0.118 / \mathrm{NLO}$ \\
$\alpha_{S}^{\text {ME }}\left(m_{\mathrm{Z}}\right)$ value/order & $0.118 / \mathrm{NLO}$ & 1.48 & 1.41 \\
MultipartonInteractions : pT0Ref $(\mathrm{GeV})$ & 1.52 & 0.02 & 0.03 \\
MultipartonInteractions : ecmPow & 0.02 & 0.60 & 0.76 \\
MultipartonInteractions : coreRadius & 0.54 & 0.30 & 0.63 \\
MultipartonInteractions : coreFraction & 0.39 & 5.61 & 5.18 \\
ColorReconnection:range & 4.73 & 0.80 & 1.04 \\
$\chi^{2} /$ dof & 0.76 & & \\
\hline
\end{tabular}

Number of MPI for various tunes

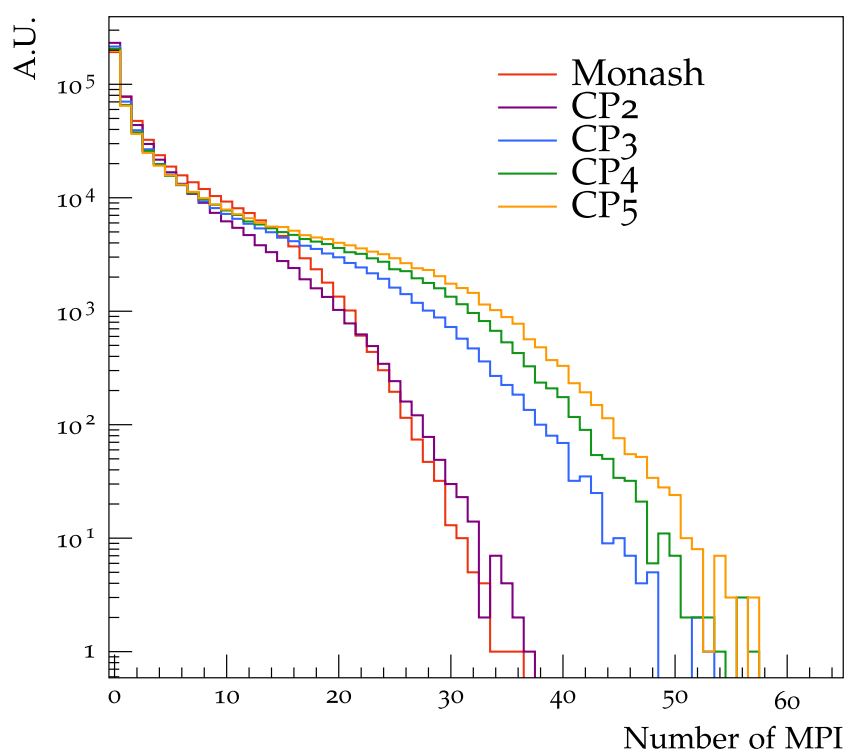

Fig. 3 Distribution of number of MPI simulated by the tunes Monash, $\mathrm{CP} 2, \mathrm{CP} 3, \mathrm{CP} 4$, and CP5 (left). Gluon distribution function at a reference scale of $\mu=3 \mathrm{GeV}$ (right) for the NNPDF2.3LO PDF set and the

related with the other UE parameters governing the MPI and $\mathrm{CR}$ contributions. In general, for a given value of the matter fraction (corefraction), MPI contributions increase for decreasing values of the core radius (coreRadius). The inclusion of the rapidity ordering for ISR in tune CP5 impacts the UE observables by reducing the number of charged particles, and needs to be compensated by a larger amount of MPI contributions.

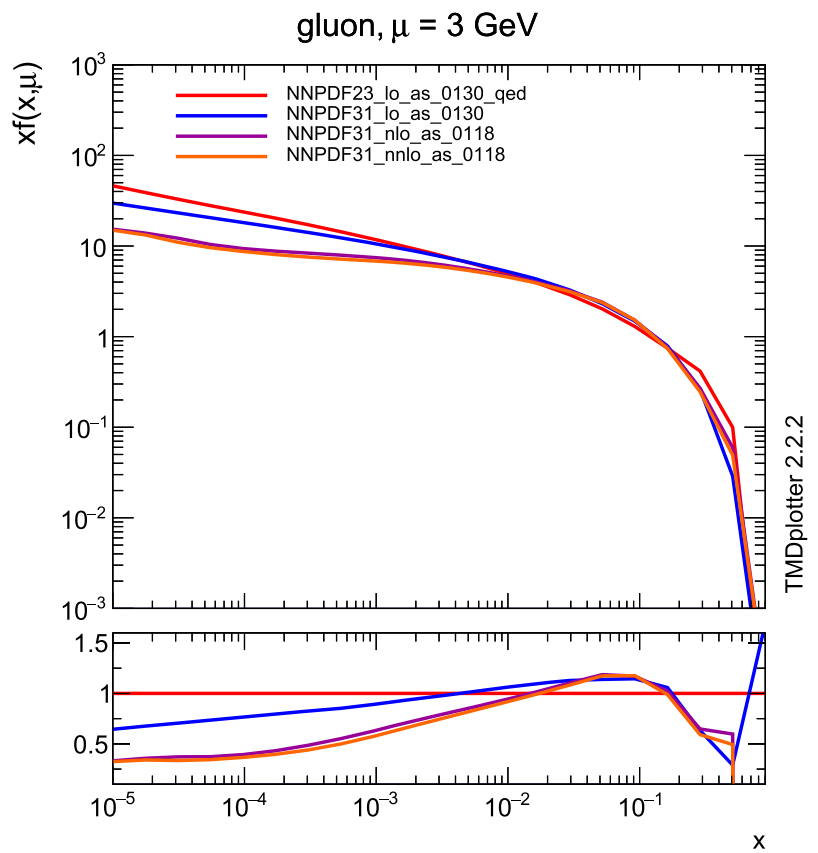

different versions of the NNPDF3.1 PDF set: LO, NLO, and NNLO. The ratio of NNPDF3.1 gluon distribution functions to the NNPDF2.3LO gluon distribution function are also shown

The $\chi^{2}$ per degree of freedom (dof) listed in Tables 2 and 3 refers to the quantity $\chi^{2}(p)$ in Eq. (1), divided by dof in the fit. The eigentunes (Appendix A) correspond to the tunes in which the changes in the $\chi^{2}\left(\Delta \chi^{2}\right)$ of the fit relative to the best fit value equals the $\chi^{2}$ value obtained in the tune, i.e., $\Delta \chi_{\min }^{2}=\chi^{2}$. Such a variation of the $\chi^{2}$ produces a tune whose uncertainty bands are roughly the same as the uncertainties in the fitted data points. This is the 
TransMIN charged-particle density, $\sqrt{s}=13 \mathrm{TeV}$

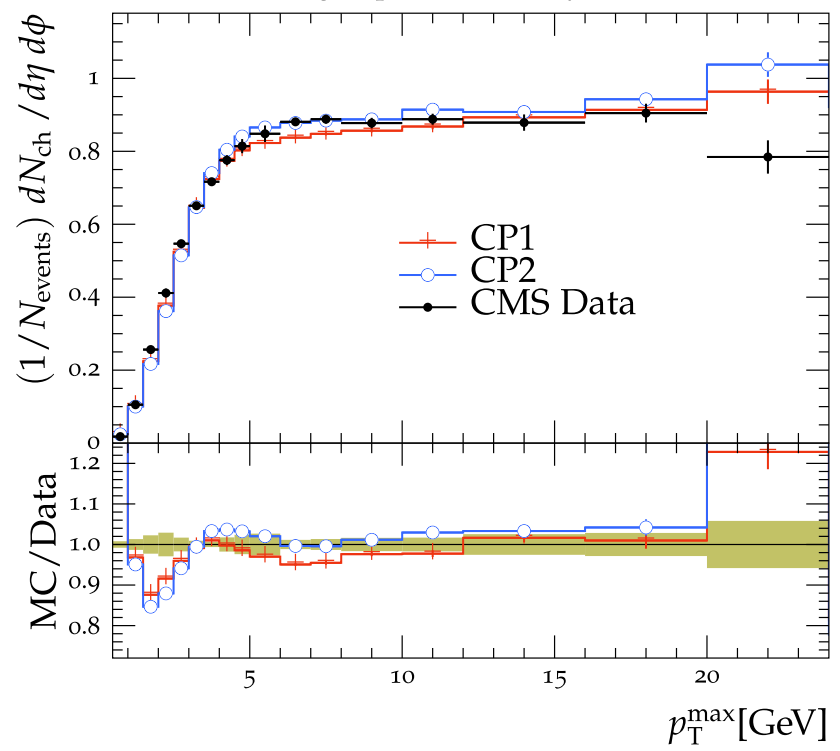

TransMAX charged-particle density, $\sqrt{s}=13 \mathrm{TeV}$

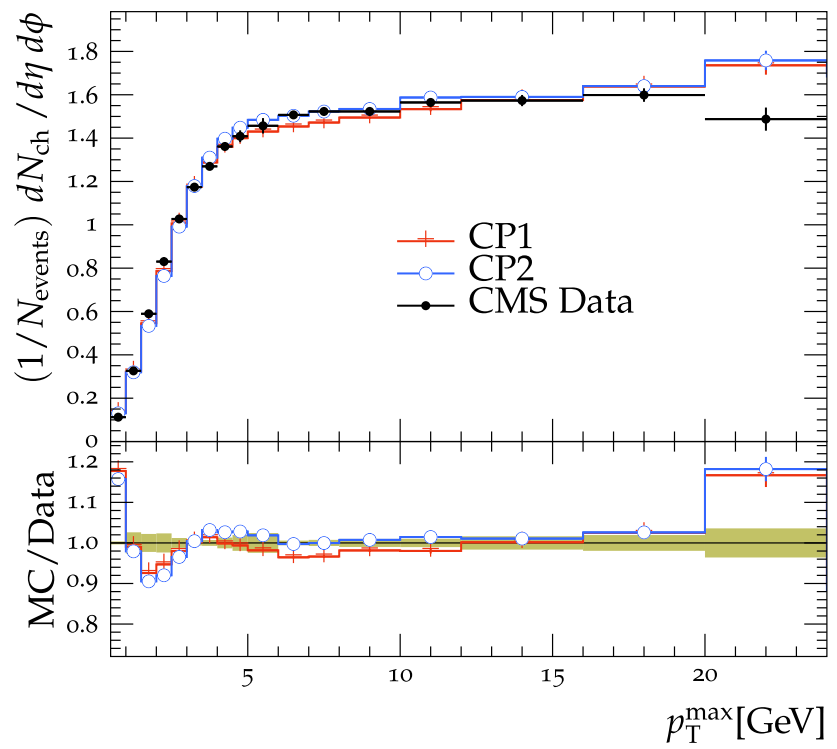

Fig. 4 The transMIN (upper left) charged-particle and charged $p_{\mathrm{T}}^{\text {sum }}$ (upper right) densities and the transMAX (lower left) charged-particle and charged $p_{\mathrm{T}}^{\text {sum }}$ (lower right) densities, as a function of the transverse momentum of the leading charged particle, $p_{\mathrm{T}}^{\max }$, from the CMS $\sqrt{s}=13 \mathrm{TeV}$ analysis [16]. Charged hadrons are measured with $p_{\mathrm{T}}>0.5 \mathrm{GeV}$ in $|\eta|<2.0$. The transMIN densities are more sensitive to the MPI, whereas the transMAX densities are more sensitive to

main motivation why this choice of variation was considered. For all tunes in Tables 2 and 3, the fit quality is good, with $\chi^{2} /$ dof values very close to 1 .

Figures 4, 5, 6 and 7 show comparisons of the UE observables measured at various collision energies to predictions from the new tunes. Figures 4 and 5 compare the chargedparticle and $p_{\mathrm{T}}^{\text {sum }}$ densities measured at $\sqrt{s}=13 \mathrm{TeV}$ by
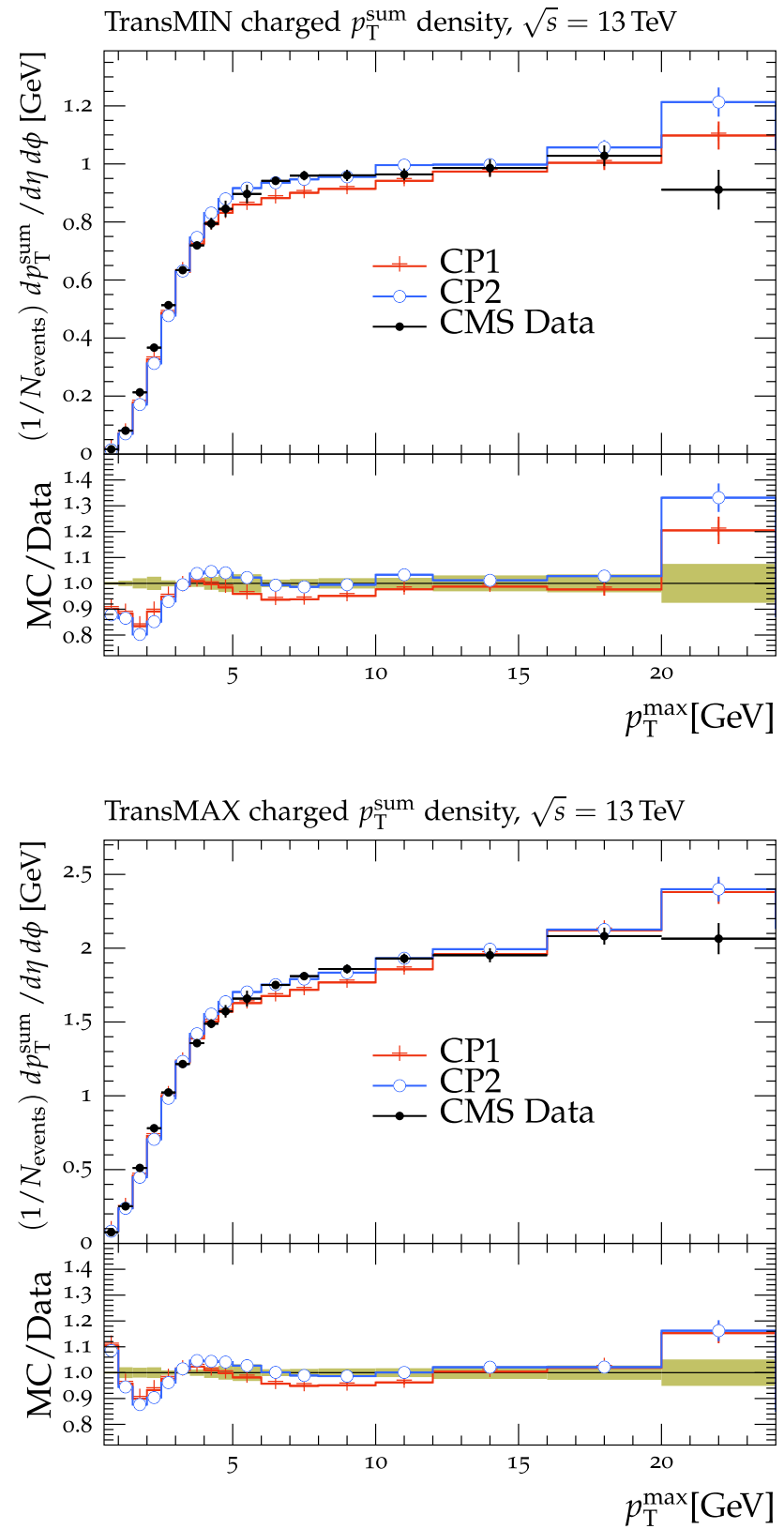

ISR and FSR. The data are compared with the CMS PYTHIA8 LO-PDF tunes $\mathrm{CP} 1$ and $\mathrm{CP} 2$. The ratios of the simulations to the data (MC/Data) are also shown, where the shaded band indicates the total experimental uncertainty in the data. Vertical lines drawn on the data points refer to the total uncertainty in the data. Vertical lines drawn on the MC points refer to the statistical uncertainty in the predictions. Horizontal bars indicate the associated bin width

the CMS experiment [16] in the transMIN and transMAX regions to predictions from the LO-PDF-based tunes and the higher-order-PDF-based tunes. Figures 6 and 7 compare the charged-particle and $p_{\mathrm{T}}^{\text {sum }}$ densities measured at $\sqrt{s}=7 \mathrm{TeV}$ by the CMS experiment [10] in the transMIN and transMAX regions to predictions from the LO-PDF-based tunes and the higher-order-PDF-based tunes. In Figs. 8 and 9 simi- 


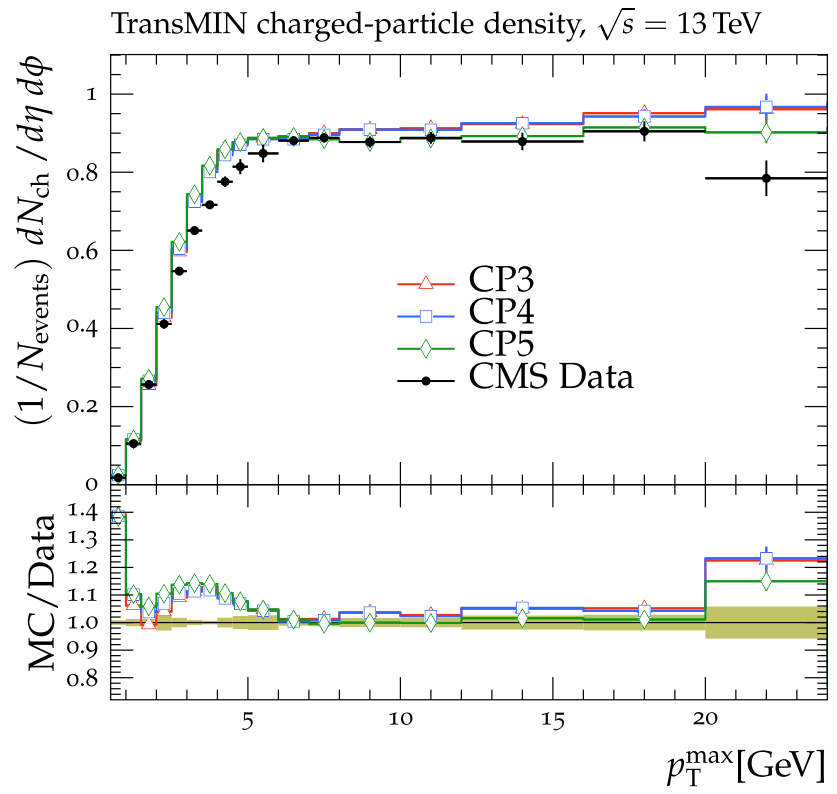

TransMAX charged-particle density, $\sqrt{s}=13 \mathrm{TeV}$

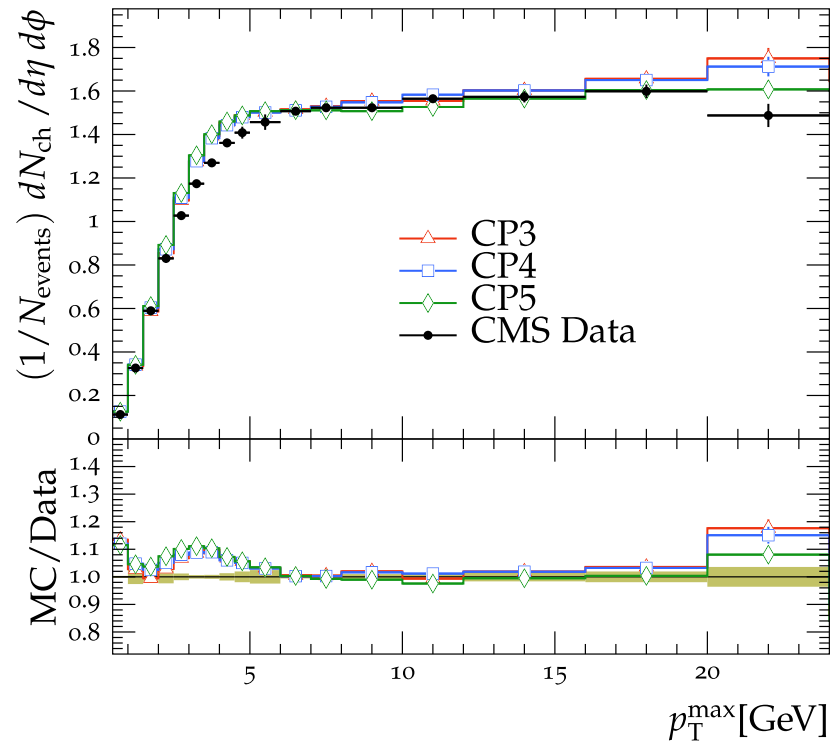

Fig. 5 The transMIN (upper left) charged-particle and charged $p_{\mathrm{T}}^{\text {sum }}$ (upper right) densities and the transMAX (lower left) charged-particle and charged $p_{\mathrm{T}}^{\text {sum }}$ (lower right) densities, as a function of the transverse momentum of the leading charged particle, $p_{\mathrm{T}}^{\max }$, from the CMS $\sqrt{s}=13 \mathrm{TeV}$ analysis [16]. Charged hadrons are measured with $p_{\mathrm{T}}>0.5 \mathrm{GeV}$ in $|\eta|<2.0$. The data are compared with the CMS

lar comparisons are shown for the observables measured at $\sqrt{s}=1.96 \mathrm{TeV}$ by the CDF experiment [9] in the transMIN and transMAX regions. All predictions reproduce well the UE observables at $\sqrt{s}=1.96,7$, and $13 \mathrm{TeV}$. Predictions from LO tunes are slightly better than the higher-order tunes in describing the energy dependence of the considered UE measurements.

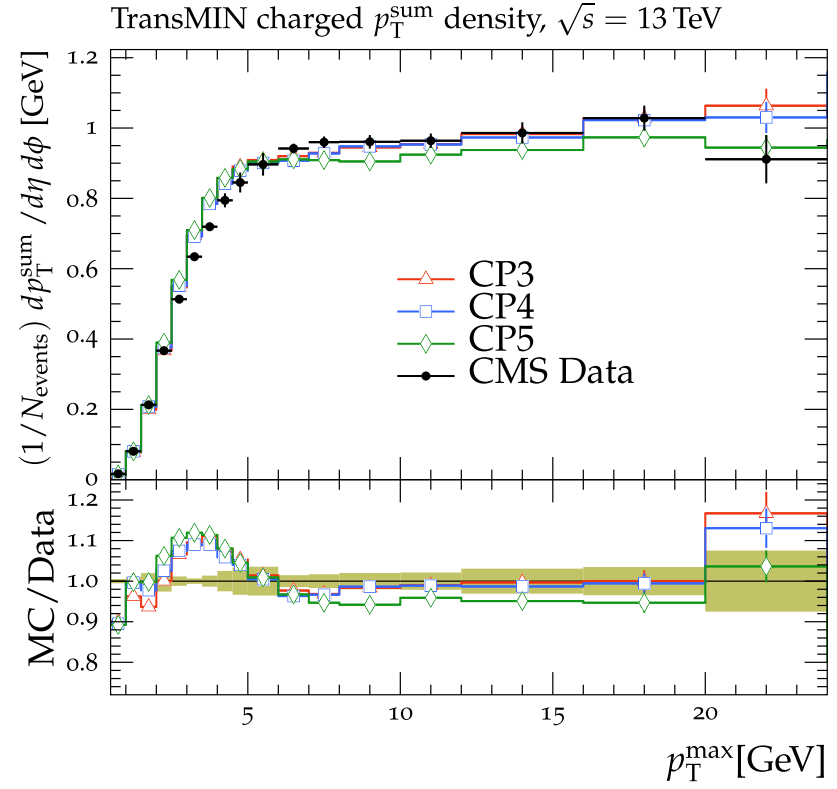

TransMAX charged $p_{\mathrm{T}}^{\text {sum }}$ density, $\sqrt{s}=13 \mathrm{TeV}$

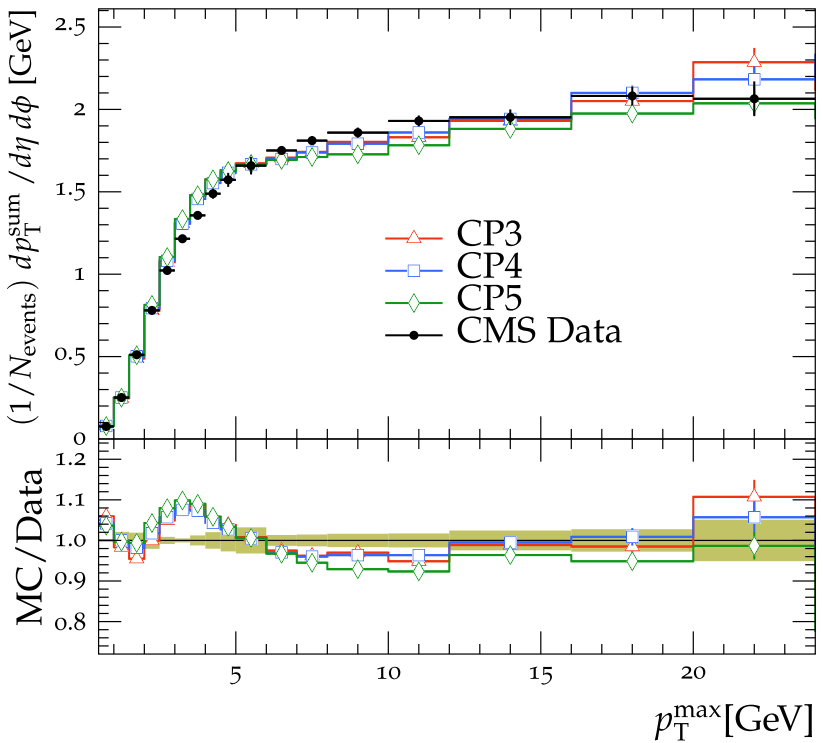

PYTHIA8 (N)NLO-PDF tunes CP3, CP4, and CP5. The ratios of simulations to the data (MC/Data) are also shown, where the shaded band indicates the total experimental uncertainty in the data. Vertical lines drawn on the data points refer to the total uncertainty in the data. Vertical lines drawn on the MC points refer to the statistical uncertainty in the predictions. Horizontal bars indicate the associated bin width

In the region of small $p_{\mathrm{T}}^{\max }$ values $\left(p_{\mathrm{T}}^{\max }<3 \mathrm{GeV}\right)$, where contributions from diffractive processes are relevant, the predictions do not always reproduce the measurements and exhibit discrepancies up to $20 \%$. Predictions from all of the new tunes cannot reproduce the UE data measured at $\sqrt{s}=300$ and $900 \mathrm{GeV}$ [9]. 
TransMIN charged-particle density, $\sqrt{s}=7 \mathrm{TeV}$

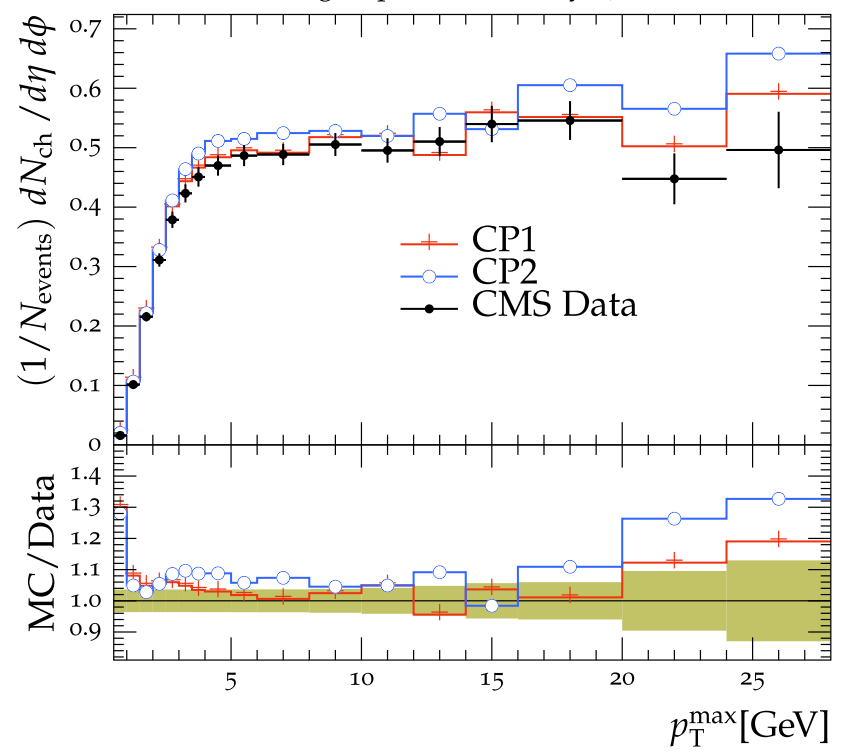

TransMAX charged-particle density, $\sqrt{s}=7 \mathrm{TeV}$

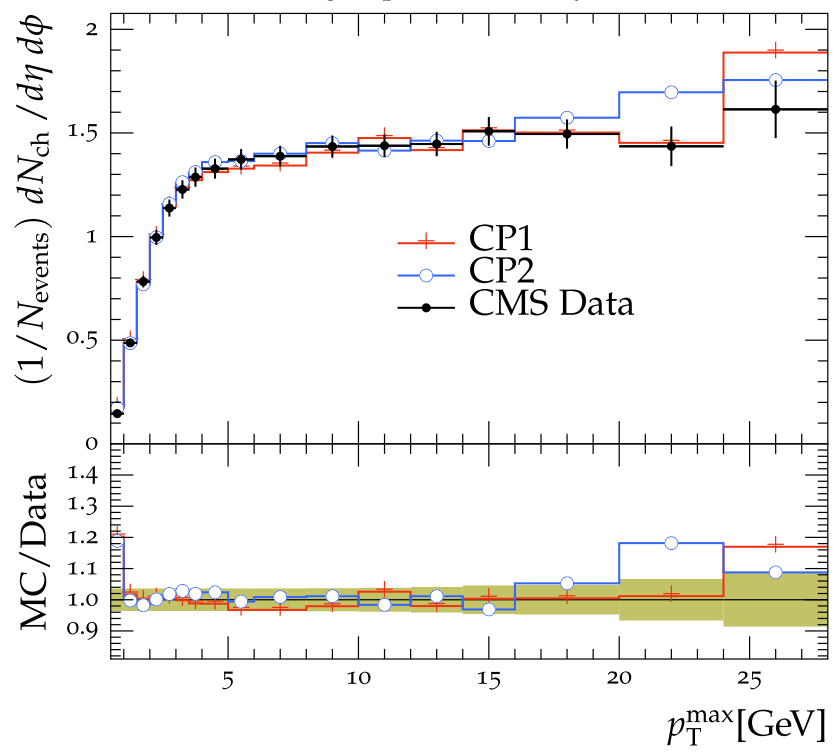

Fig. 6 The transMIN (upper left) charged-particle and charged $p_{\mathrm{T}}^{\text {sum }}$ (upper right) densities and the transMAX (lower left) charged-particle and charged $p_{\mathrm{T}}^{\text {sum }}$ (lower right) densities, as a function of the transverse momentum of the leading charged particle, $p_{\mathrm{T}}^{\max }$, from the CMS $\sqrt{s}=7 \mathrm{TeV}$ analysis [10]. Charged hadrons are measured with $p_{\mathrm{T}}>0.5 \mathrm{GeV}$ in $|\eta|<0.8$. The data are compared with the CMS

Figure 10 shows the charged-particle multiplicity as a function of pseudorapidity for charged particles in $|\eta|<2$ measured by the CMS experiment at $\sqrt{s}=13 \mathrm{TeV}$ [18] in $\mathrm{MB}$ events. These events were recorded with no magnetic field, so all particles irrespective of their $p_{\mathrm{T}}$ are measured. Data are compared with the predictions of the new PYTHIA8
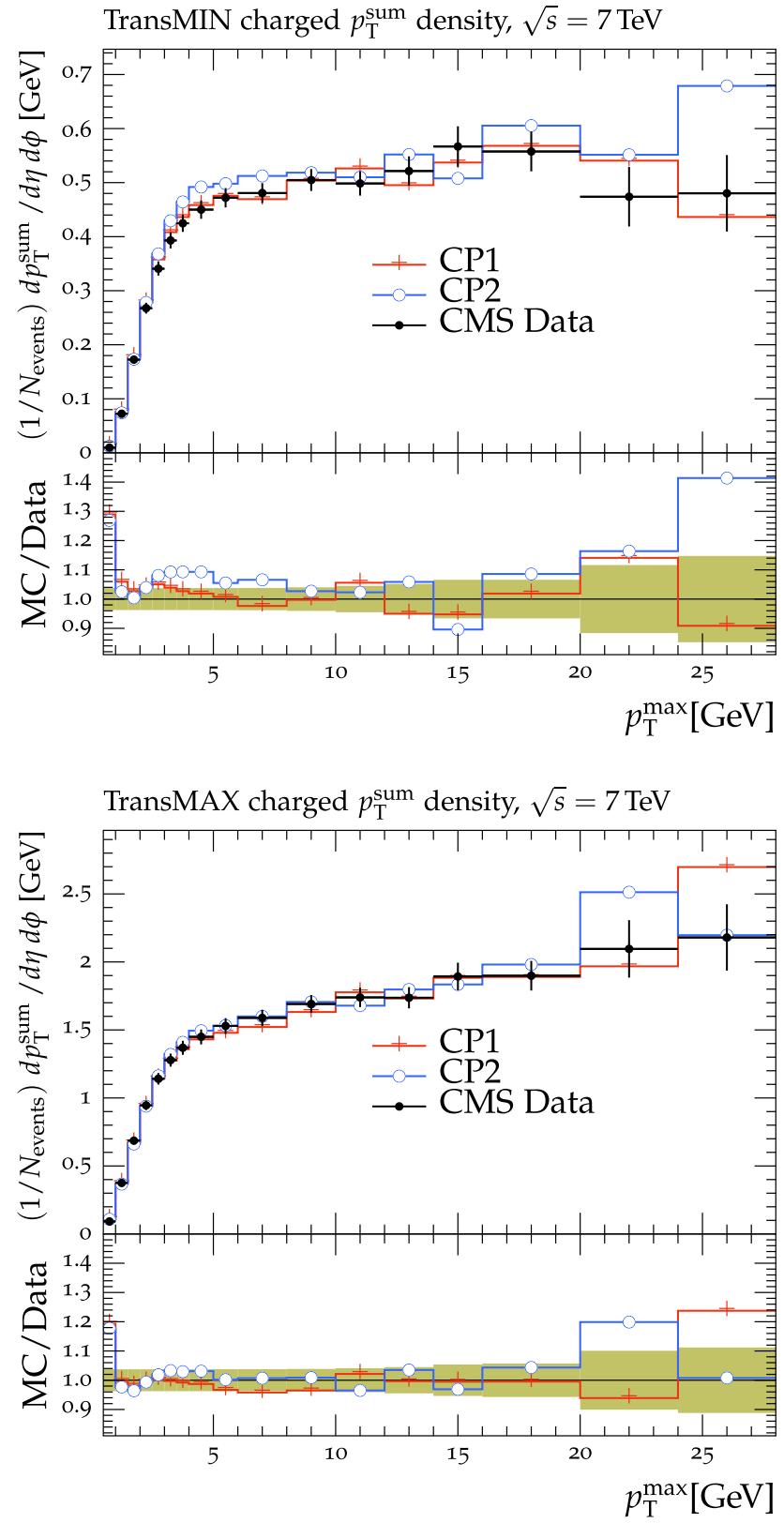

PYTHIA8 LO-PDF tunes CP1 and CP2. The ratios of simulations to the data (MC/Data) are also shown, where the shaded band indicates the total experimental uncertainty in the data. Vertical lines drawn on the data points refer to the total uncertainty in the data. Vertical lines drawn on the MC points refer to the statistical uncertainty in the predictions. Horizontal bars indicate the associated bin width

tunes. All of them are able to reproduce the measurement at the same level of agreement, independently of the PDF used for the UE simulation. We could not find any MB or UE observable where the level of agreement between data and predictions from the different tunes is significantly different. 


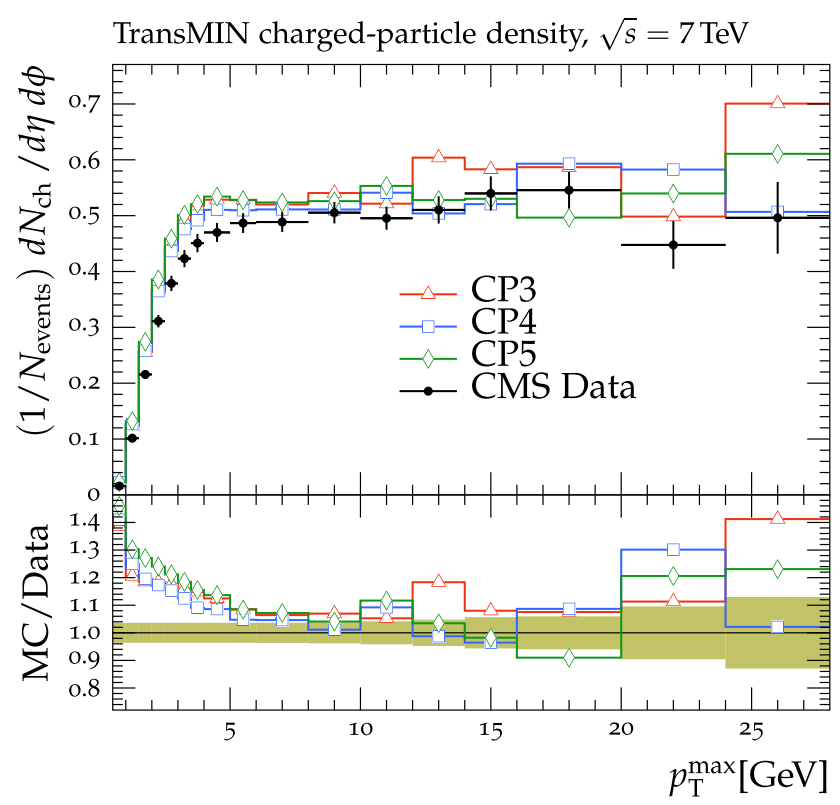

TransMAX charged-particle density, $\sqrt{s}=7 \mathrm{TeV}$

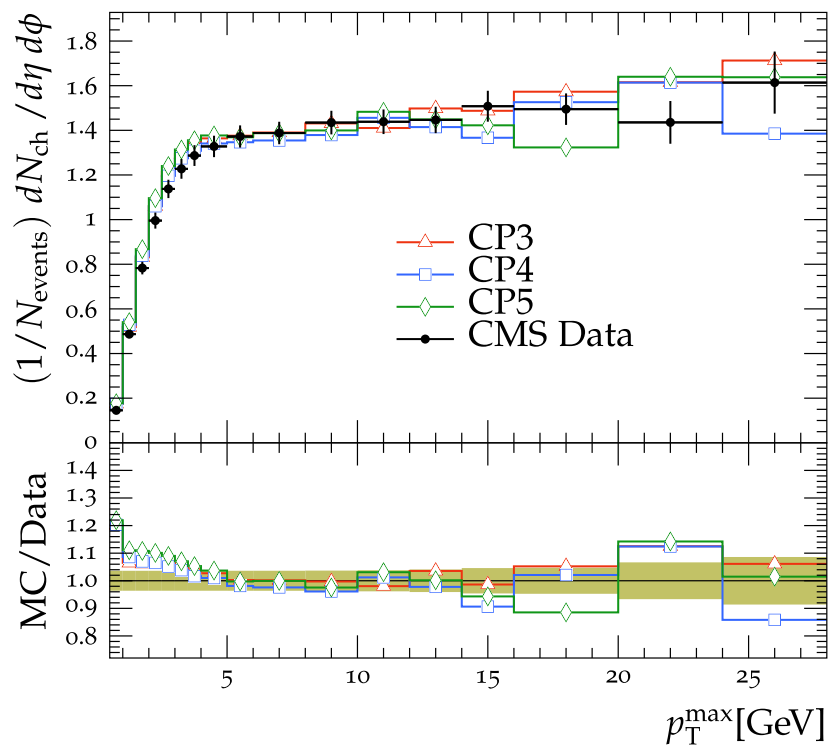

Fig. 7 The transMIN (upper left) charged-particle and charged $p_{\mathrm{T}}^{\text {sum }}$ (upper right) densities and the transMAX (lower left) charged-particle and charged $p_{\mathrm{T}}^{\text {sum }}$ (lower right) densities, as a function of the transverse momentum of the leading charged particle, $p_{\mathrm{T}}^{\max }$, from the CMS $\sqrt{s}=7 \mathrm{TeV}$ analysis [10]. Charged hadrons are measured with $p_{\mathrm{T}}>0.5 \mathrm{GeV}$ in $|\eta|<0.8$. The data are compared with the CMS

\section{Validation of the new PYTHIA8 tunes}

In this section, comparisons of the predictions obtained with the new tunes to various experimental measurements performed by the CMS experiment are provided. Unless otherwise stated, the comparisons are made at $\sqrt{s}=13 \mathrm{TeV}$. We compare the CMS UE tunes with MB and UE data measured
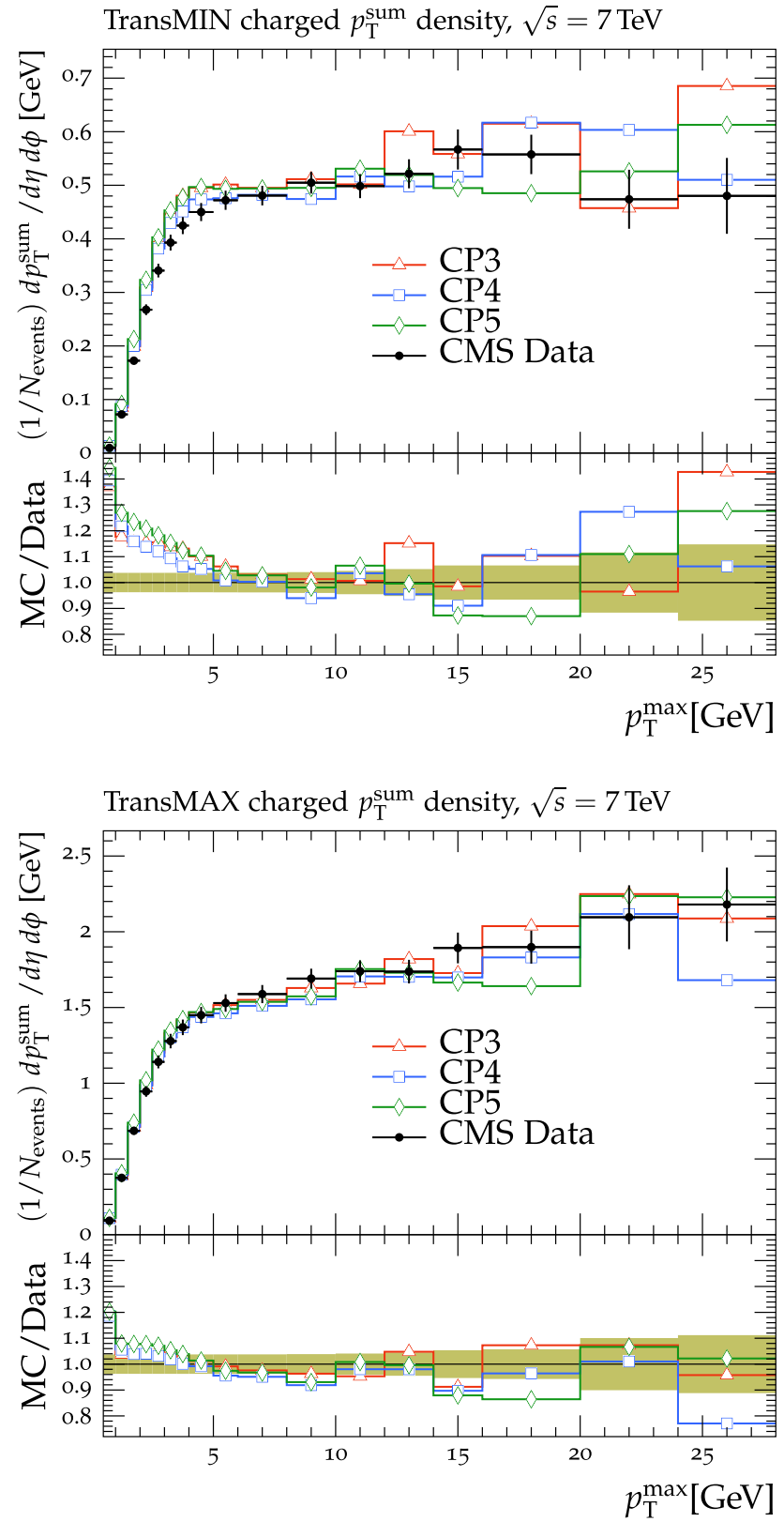

PYTHIA8 (N)NLO-PDF tunes CP3, CP4, and CP5. The ratios of simulations to the data (MC/Data) are also shown, where the shaded band indicates the total experimental uncertainty in the data. Vertical lines drawn on the data points refer to the total uncertainty in the data. Vertical lines drawn on the MC points refer to the statistical uncertainty in the predictions. Horizontal bars indicate the associated bin width

at central and forward pseudorapidities that are not used in the fits. We examine how well multijet, Drell-Yan, and top quark observables are predicted by MC simulations using higher-order ME generators merged with PYTHIA8 with the various new tunes. 
TransMIN charged-particle density, $\sqrt{s}=1.96 \mathrm{TeV}$

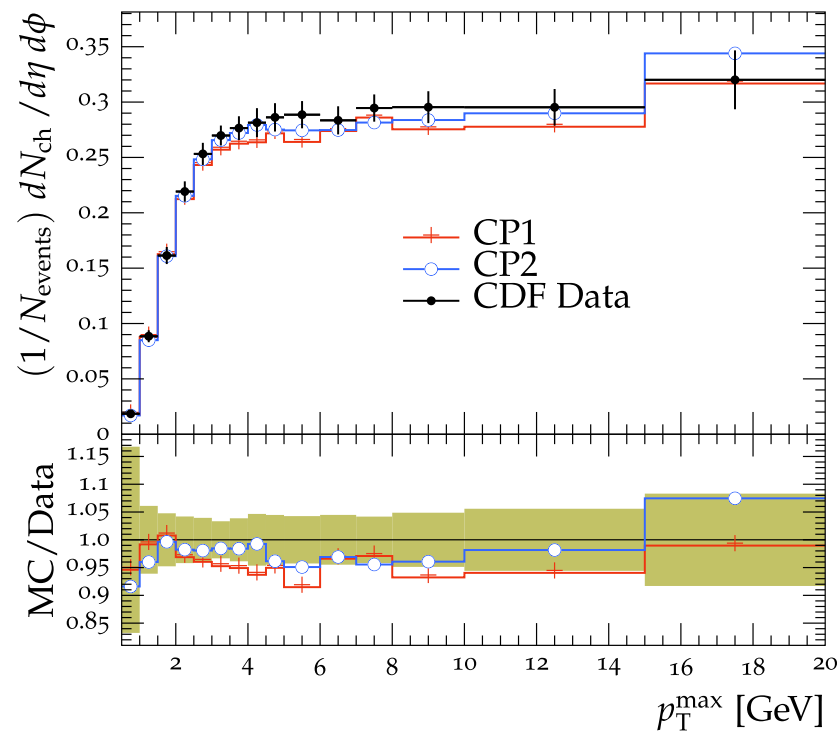

TransMAX charged-particle density, $\sqrt{s}=1.96 \mathrm{TeV}$

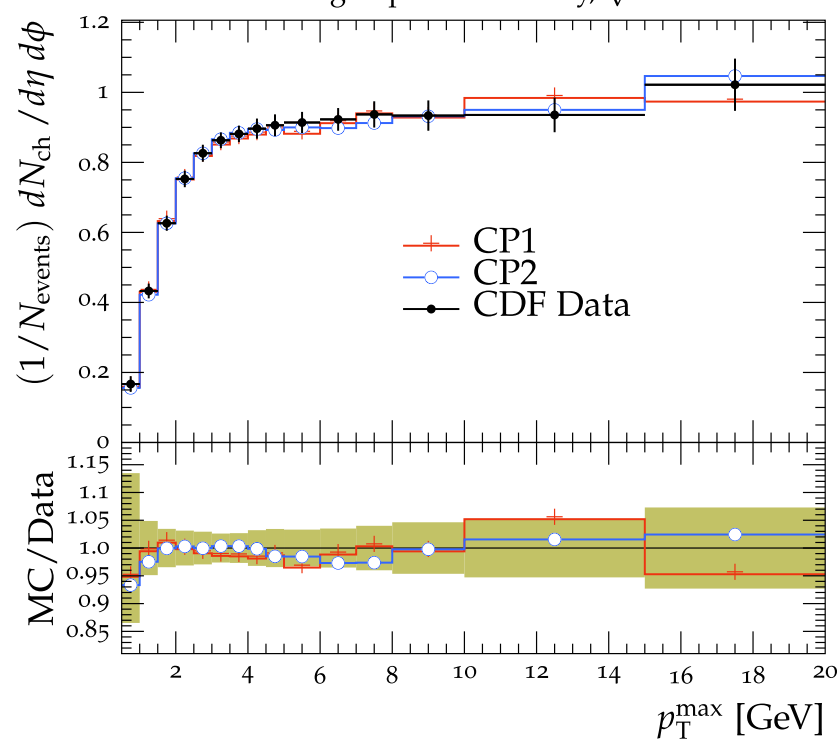

Fig. 8 The transMIN (upper left) charged-particle and charged $p_{\mathrm{T}}^{\text {sum }}$ (upper right) densities and the transMAX (lower left) charged-particle and charged $p_{\mathrm{T}}^{\text {sum }}$ (lower right) densities, as a function of the transverse momentum of the leading charged particle, $p_{\mathrm{T}}^{\max }$, from the CDF $\sqrt{s}=1.96 \mathrm{TeV}$ analysis [9]. Charged hadrons are measured with $p_{\mathrm{T}}>0.5 \mathrm{GeV}$ in $|\eta|<0.8$. The data are compared with the CMS

\subsection{Comparisons using event-shape observables}

In this subsection, predictions of the new tunes are compared to event-shape observables measured at LEP, in electronpositron collisions. These observables are particularly sensitive to the value of $\alpha_{S}^{\mathrm{FSR}}\left(m_{\mathrm{Z}}\right)$. Given the leptonic initial state, there is no effect coming from the values of the MPI, color reconnection, and ISR parameters.
TransMIN charged $p_{\mathrm{T}}^{\text {sum }}$ density, $\sqrt{s}=1.96 \mathrm{TeV}$

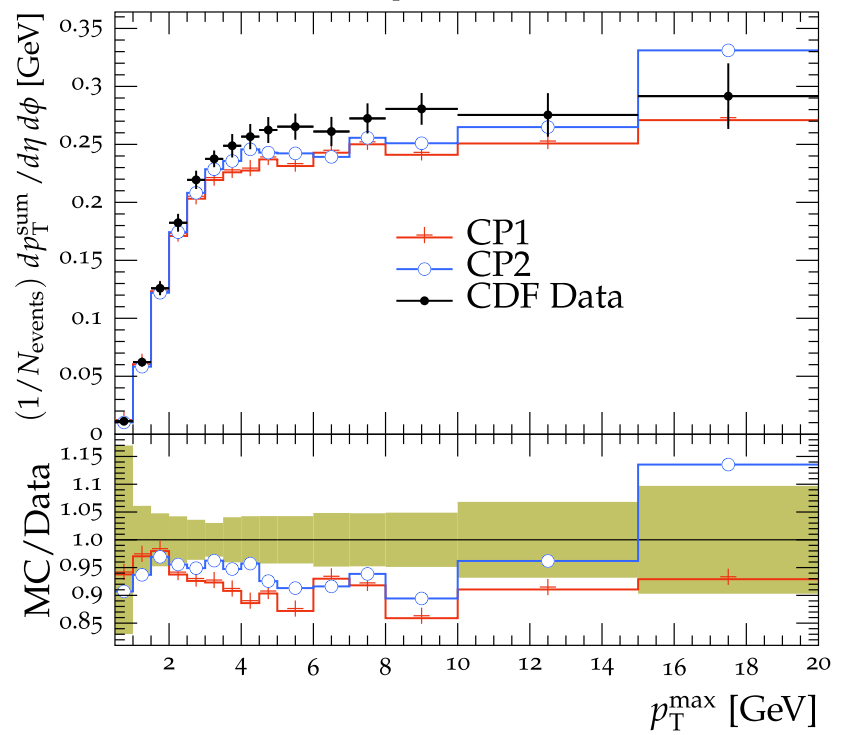

TransMAX charged $p_{\mathrm{T}}^{\text {sum }}$ density, $\sqrt{s}=1.96 \mathrm{TeV}$

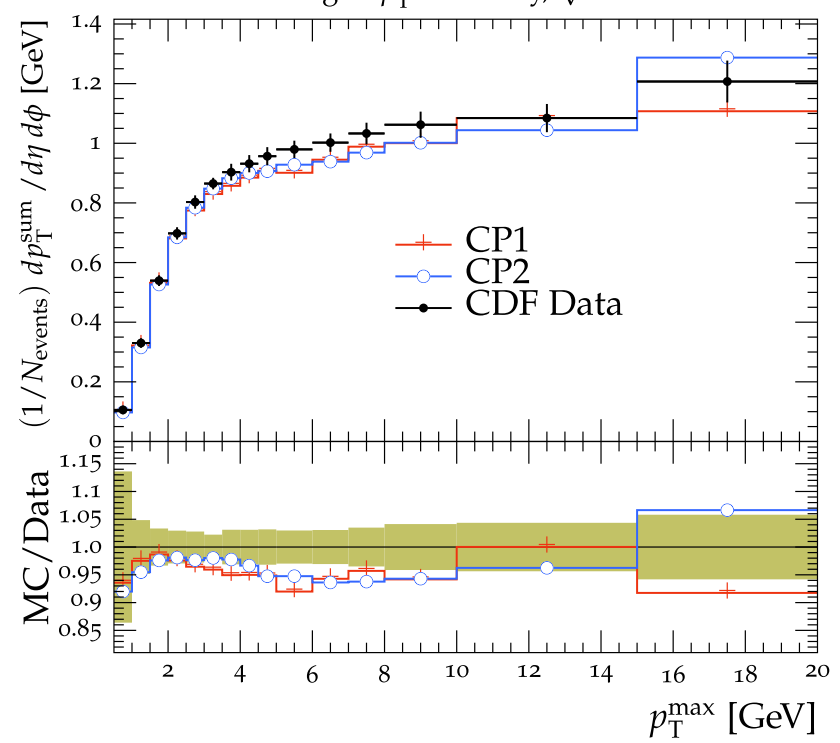

PYTHIA8 LO-PDF tunes CP1 and CP2. The ratios of simulations to the data (MC/Data) are also shown, where the shaded band indicates the total experimental uncertainty in the data. Vertical lines drawn on the data points refer to the total uncertainty in the data. Vertical lines drawn on the MC points refer to the statistical uncertainty in the predictions. Horizontal bars indicate the associated bin width

When predictions with PYTHIA 8 are used, an optimal value of $\alpha_{S}^{\mathrm{FSR}}\left(m_{\mathrm{Z}}\right) \sim 0.13$ is found, which best describes these observables, independent of the PDF used for the modeling of the PS evolution.

Figures 11 and 12 display the oblateness $(O)$, sphericity $(S)$, thrust $(T)$, and thrust major $\left(T_{\text {major }}\right)$, measured in $\mathrm{e}^{+} \mathrm{e}^{-} \rightarrow \mathrm{Z} \gamma^{*} \rightarrow \mathrm{q} \overline{\mathrm{q}}$ final states at $\sqrt{s}=91.2 \mathrm{GeV}$ by the ALEPH experiment [45]. These observables measure the 


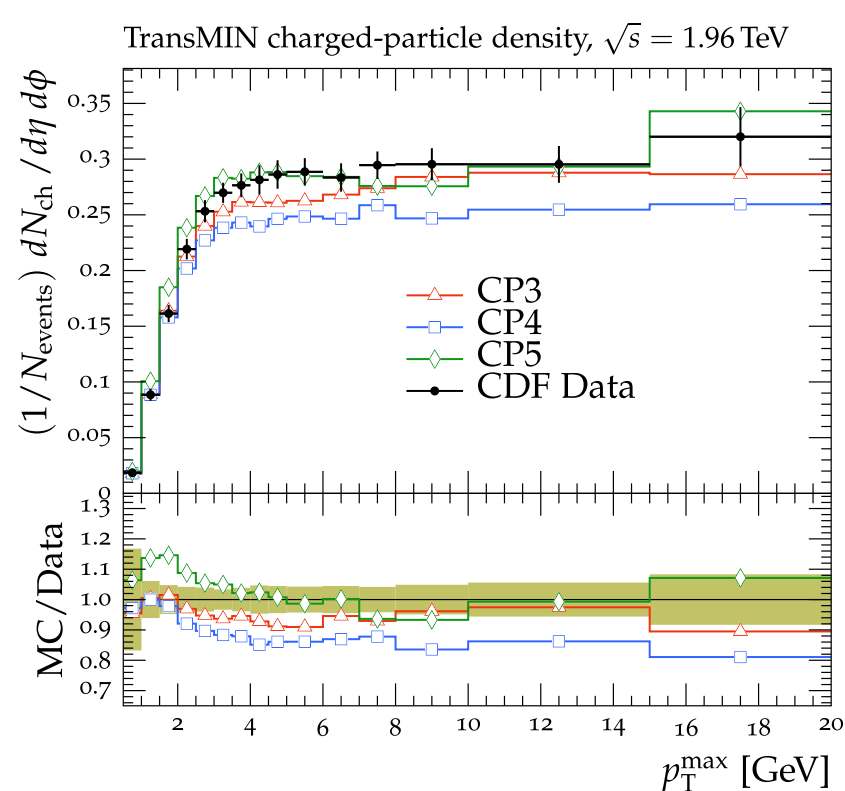

TransMAX charged-particle density, $\sqrt{s}=1.96 \mathrm{TeV}$

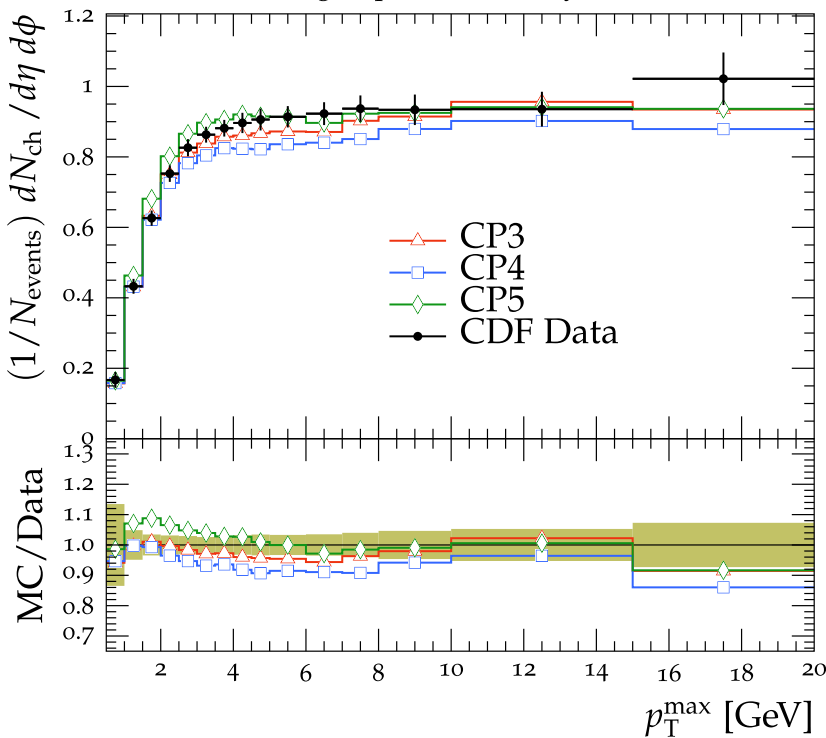

Fig. 9 The transMIN (upper left) charged-particle and charged $p_{\mathrm{T}}^{\text {sum }}$ (upper right) densities and the transMAX (lower left) charged-particle and charged $p_{\mathrm{T}}^{\text {sum }}$ (lower right) densities, as a function of the transverse momentum of the leading charged particle, $p_{\mathrm{T}}^{\max }$, from the CDF $\sqrt{s}=1.96 \mathrm{TeV}$ analysis [9]. Charged hadrons are measured with $p_{\mathrm{T}}>0.5 \mathrm{GeV}$ in $|\eta|<0.8$. The data are compared with the CMS

topology of the event. An isotropic event would have a value of $T$ close to 0.5 , while values of $T$ close to 1 correspond to 2-jet events.

Predictions obtained with MG5_aMC with up to 4 partons in the final state, and interfaced with the UE from the tune CUETP8M1 and the new PYTHIA 8 tunes CP2, CP3, and CP5 are considered (Fig. 11). Predictions using the tune CP2 do not describe the event-shape observables very well,
TransMIN charged $p_{\mathrm{T}}^{\text {sum }}$ density, $\sqrt{s}=1.96 \mathrm{TeV}$

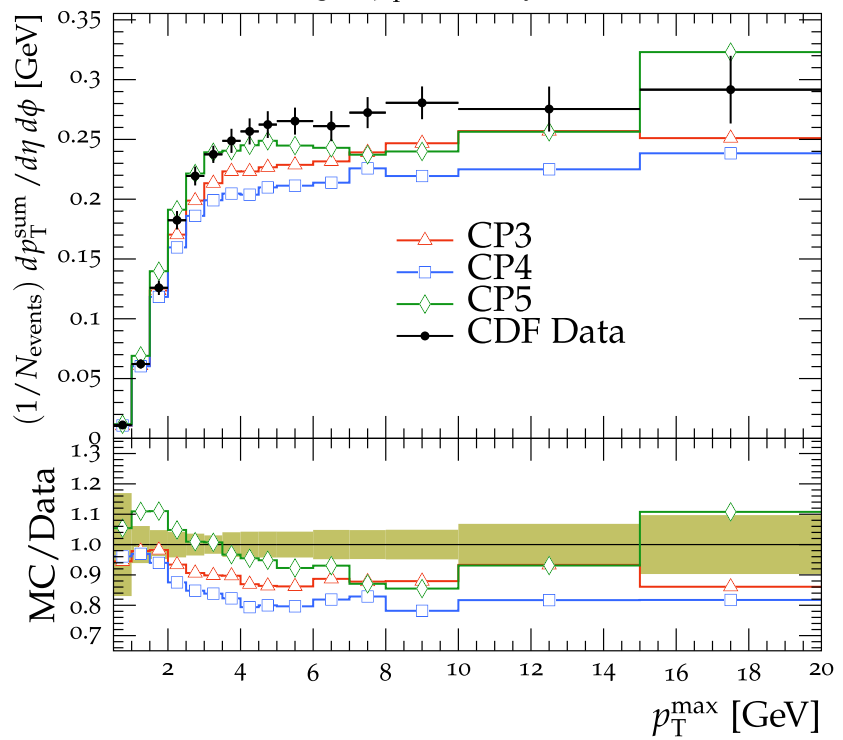

TransMAX charged $p_{\mathrm{T}}^{\text {sum }}$ density, $\sqrt{s}=1.96 \mathrm{TeV}$

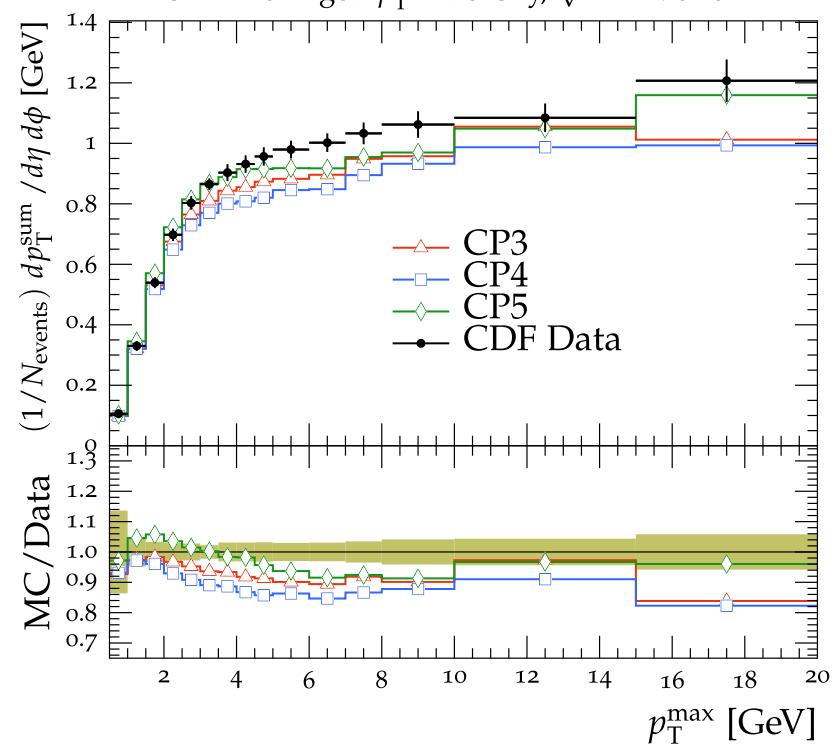

PYTHIA8 (N)NLO-PDF tunes CP3, CP4, and CP5. The ratios of simulations to the data (MC/Data) are also shown, where the shaded band indicates the total experimental uncertainty in the data. Vertical lines drawn on the data points refer to the total uncertainty in the data. Vertical lines drawn on the MC points refer to the statistical uncertainty in the predictions. Horizontal bars indicate the associated bin width

with discrepancies with the data up to $30 \%$ in the $T$ and $T_{\text {major }}$ In particular, tune CP2 predicts too many isotropical events. A similar description is obtained for predictions of MG5_aMC+PYTHIA 8 with the tune CUETP8M1. A better agreement in the event-shape variables is observed for predictions using tune CP3 and CP5. A correct description of event-shape observables strongly depends on the value of the FSR strong coupling. The observations above indi- 


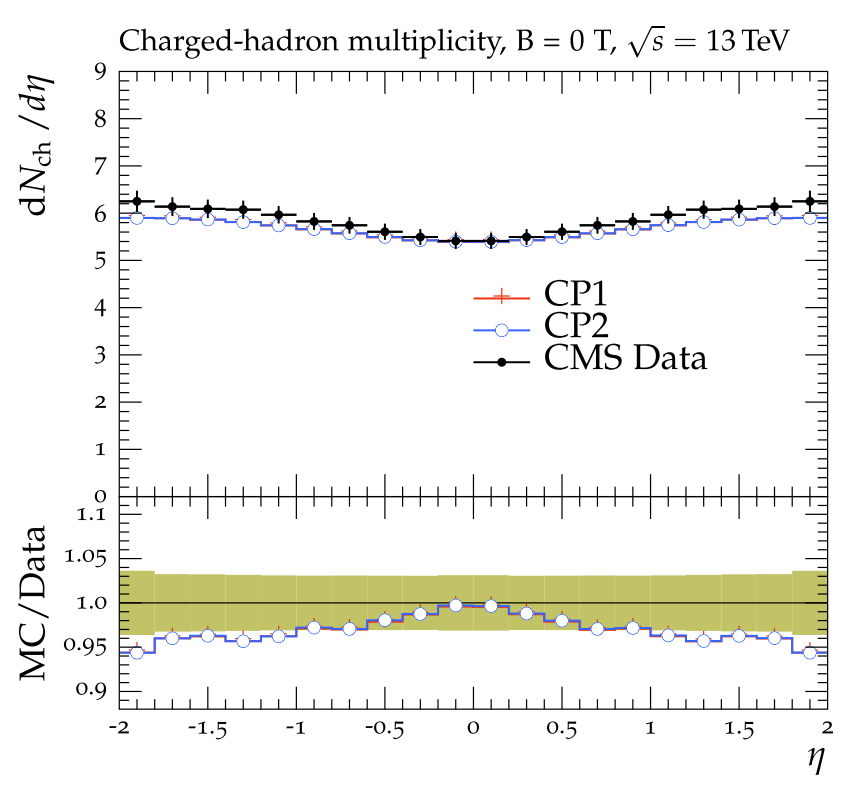

Fig. 10 The pseudorapidity distribution of charged hadrons measured in $|\eta|<2$ for an inclusive selection in inelastic proton-proton collisions, with zero magnetic field strength $(\mathrm{B}=0 \mathrm{~T})$, from the $\mathrm{CMS} \sqrt{s}=13 \mathrm{TeV}$ analysis [18]. The data are compared with the CMS PYTHIA8 LO-PDF tunes CP1 and CP2 (left), and with the CMS PYTHIA8 NLO-PDF tune CP3 and the CMS PYTHIA8 NNLO-PDF tunes CP4 and CP5 (right).

cate that when merged configurations are considered, i.e., MG5_aMC + PYTHIA8, where partons at higher multiplicities in the final state are simulated at the ME level, the description of event-shape observables degrades. A value of $\alpha_{S}^{\mathrm{FSR}}\left(m_{\mathrm{Z}}\right) \sim 0.13$ generally overestimates the number of final-state partons, while a lower $\alpha_{S}^{\mathrm{FSR}}\left(m_{\mathrm{Z}}\right) \sim 0.12$ performs better.

At large values of $T$, where the hadronization effects become relevant, we observe a large difference between predictions from tunes using a small $\alpha_{S}^{\mathrm{FSR}}$ (CP3 and CP5) and tunes using a large $\alpha_{S}^{\mathrm{FSR}}$ (CP2 and CUETP8M1). These differences may be due to the interplay between the value of the strong coupling and the hadronization. Analyses particularly sensitive to hadronization should carefully evaluate the corresponding systematic uncertainties. In some cases retuning hadronization parameters may be desired.

We also compared MG5_aMC+PYTHIA 8 with CP5, and CP5 with CMW rescaling [46] (Fig. 12). Apart from $T$, for all shape variables considered, CP5 without CMW rescaling describes the data better.

\subsection{Comparisons using MB and other UE observables}

In this subsection, predictions of the new tunes are compared to the observables measured in MB collisions that are sensitive to contributions from soft emissions and MPI. Figure 13 shows the charged-particle multiplicity as a function of pseudorapidity [24] in NSD-enhanced and SD-enhanced event

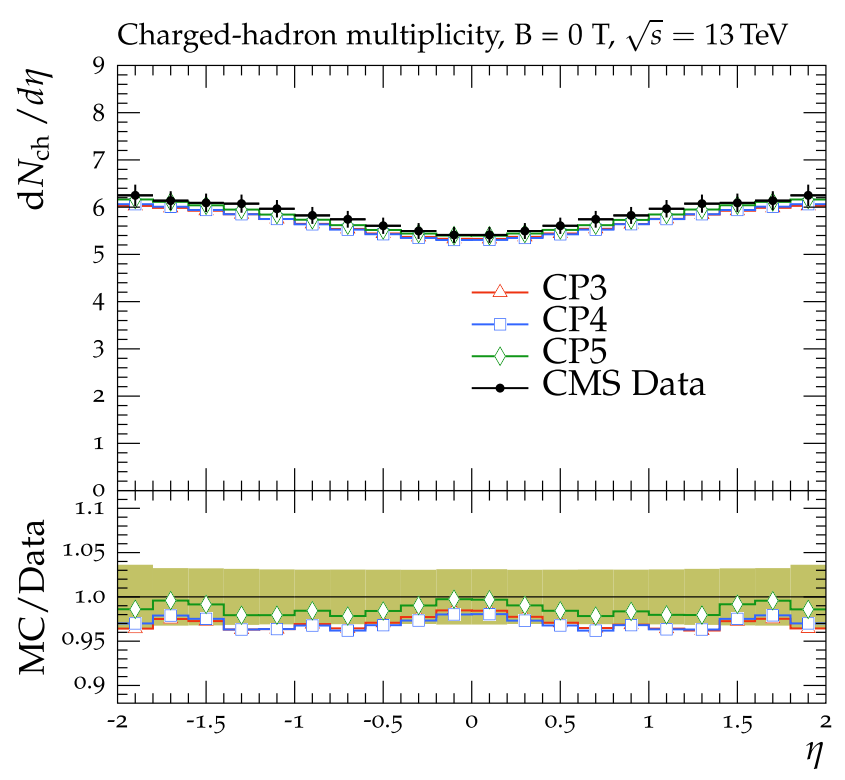

The ratios of simulations to the data (MC/Data) are also shown, where the shaded band indicates the total experimental uncertainty in the data. Vertical lines drawn on the data points refer to the total uncertainty in the data. Vertical lines drawn on the MC points refer to the statistical uncertainty in the predictions. Horizontal bars indicate the associated bin width

samples. The details of the selections can be found in Ref. [24]. These observables are sensitive to SD, CD, and DD dissociation. It is observed that predictions from all of the tunes are similar to each other and describe well the measurements for both considered selections. This shows that the number of charged particles produced in diffractive processes and inelastic collisions is simultaneously described by the new CMS tunes. Figure 13 also demonstrates that tunes based on NNPDF3.1 PDF sets at orders higher than LO adequately describe the MB data.

Figure 14 shows the UE observables, i.e., charged-particle multiplicity and $p_{\mathrm{T}}^{\text {sum }}$ densities [16], as a function of the $p_{\mathrm{T}}$ of the leading jet reconstructed using just charged particles. The observables shown in Fig. 14 are from events selected without requiring any NSD- or SD-enhanced selections. The CMS UE tunes describe well UE-sensitive data measured using the leading charged-particle jet for $p_{\mathrm{T}}{ }^{\text {jet }}>10 \mathrm{GeV}$. Tunes based on NLO or NNLO PDF sets, i.e., CP3, CP4, and CP5, describe the region at lower $p_{\mathrm{T}}{ }^{\text {jet }}$ better than CP1 and CP2, which are based on LO PDF sets. Predictions obtained with $\mathrm{CP} 1$ and $\mathrm{CP} 2$ underestimate the UE observables by about $\approx 15-20 \%$. Predictions obtained with CP3, CP4, and CP5 describe the UE in events characterized using the leading charged particle, as well as those characterized by the leading charged-particle jet, quite well.

Predictions for observables measured in the forward region are compared with data and shown in Figs. 15 and 16. The energy flow, defined as the average energy per event [47], 
Oblateness $\left(E_{\mathrm{CMS}}=91.2 \mathrm{GeV}\right)$

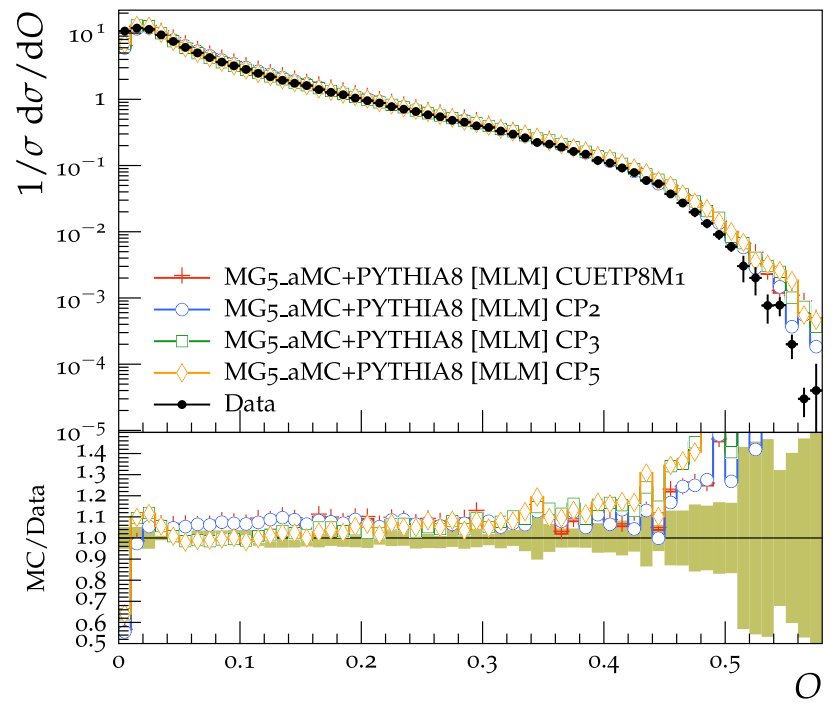

Thrust major $\left(E_{\mathrm{CMS}}=91.2 \mathrm{GeV}\right)$

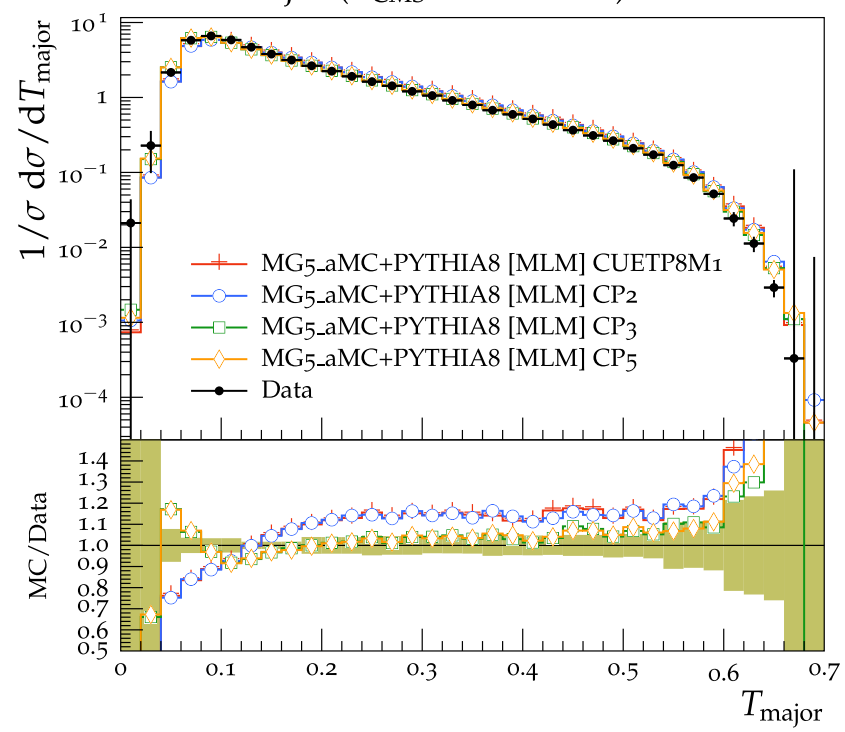

Fig. 11 The normalized cross sections as a function of event-shape variables, oblateness (upper left), sphericity (upper right), thrust (lower left), and thrust major (lower right) from the ALEPH $\sqrt{s}=91.2 \mathrm{GeV}$ analysis [45], compared with the predictions by MG5_aMC + PYTHIA8 with $k_{\mathrm{T}}-\mathrm{MLM}$ merging, for tunes $\mathrm{CP} 2, \mathrm{CP} 3$, and $\mathrm{CP} 5$. The ratio of

as a function of $\eta$ with the Hadron Forward (HF) calorimeter [48] covering $3.15<|\eta|<5.20$ and the CASTOR calorimeter [48] covering $-6.6<\eta<-5.2$, is well reproduced by all tunes. A different level of agreement is achieved for predictions from the new CMS tunes for the spectrum of the total energy E measured in the CASTOR calorimeter at $\sqrt{s}=13 \mathrm{TeV}$ [49], displayed in Fig. 16. In particular, the tunes based on LO PDF sets reproduce the energy spectrum well at large values $(\mathrm{E}>2000 \mathrm{GeV})$, but have differences of up to $40 \%$ at low values $(\mathrm{E}<800 \mathrm{GeV})$. The tunes using

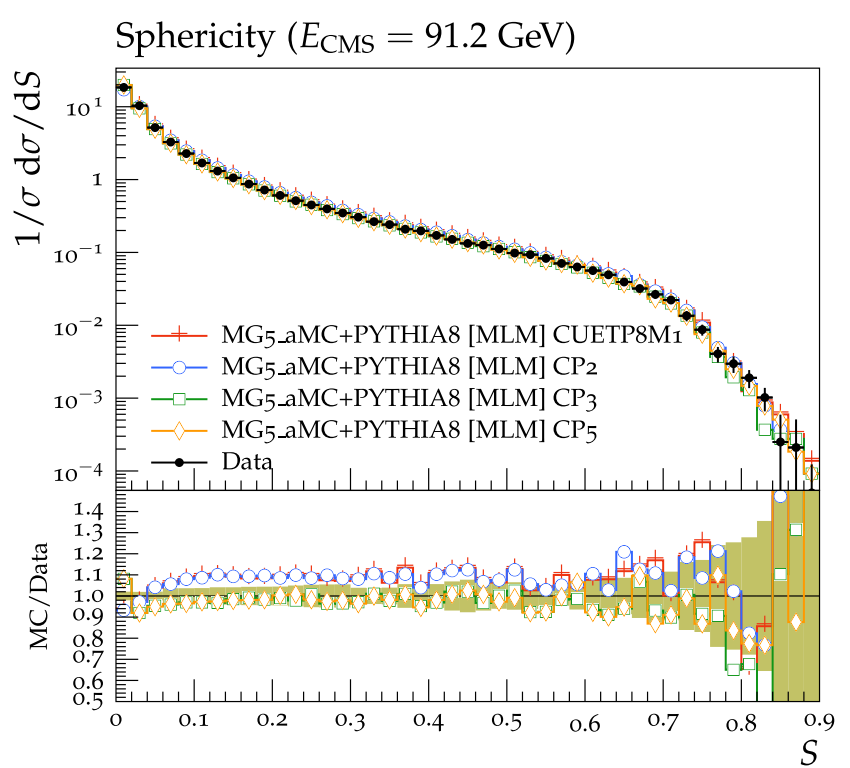

Thrust $\left(E_{\mathrm{CMS}}=91.2 \mathrm{GeV}\right)$

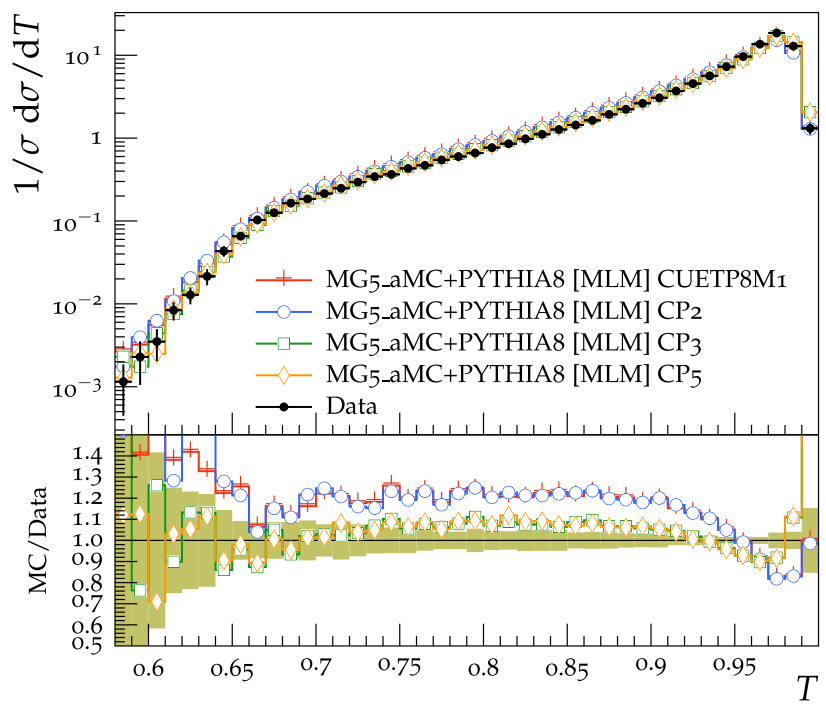

the simulations to the data (MC/Data) is also shown, where the shaded band indicates the total experimental uncertainty in the data. Vertical lines drawn on the data points refer to the total uncertainty in the data. Vertical lines drawn on the MC points refer to the statistical uncertainty in the predictions. Horizontal bars indicate the associated bin width

higher-order PDF sets are closer to the data at low energy values, with differences up to $20 \%$, but tend to overestimate the energy at large values. This dissimilar behaviour is driven by the different pTORef values of the tunes. The fiducial inelastic cross sections [50], when two different selections are applied in the forward region, are not well reproduced by any of the new tunes or by CUETP8M1, with differences up to $10 \%$. This might be because of the Schüler-Sjöstrand [44] diffraction model used in the simulation, which might have a suboptimal description of the low-mass diffractive compo- 

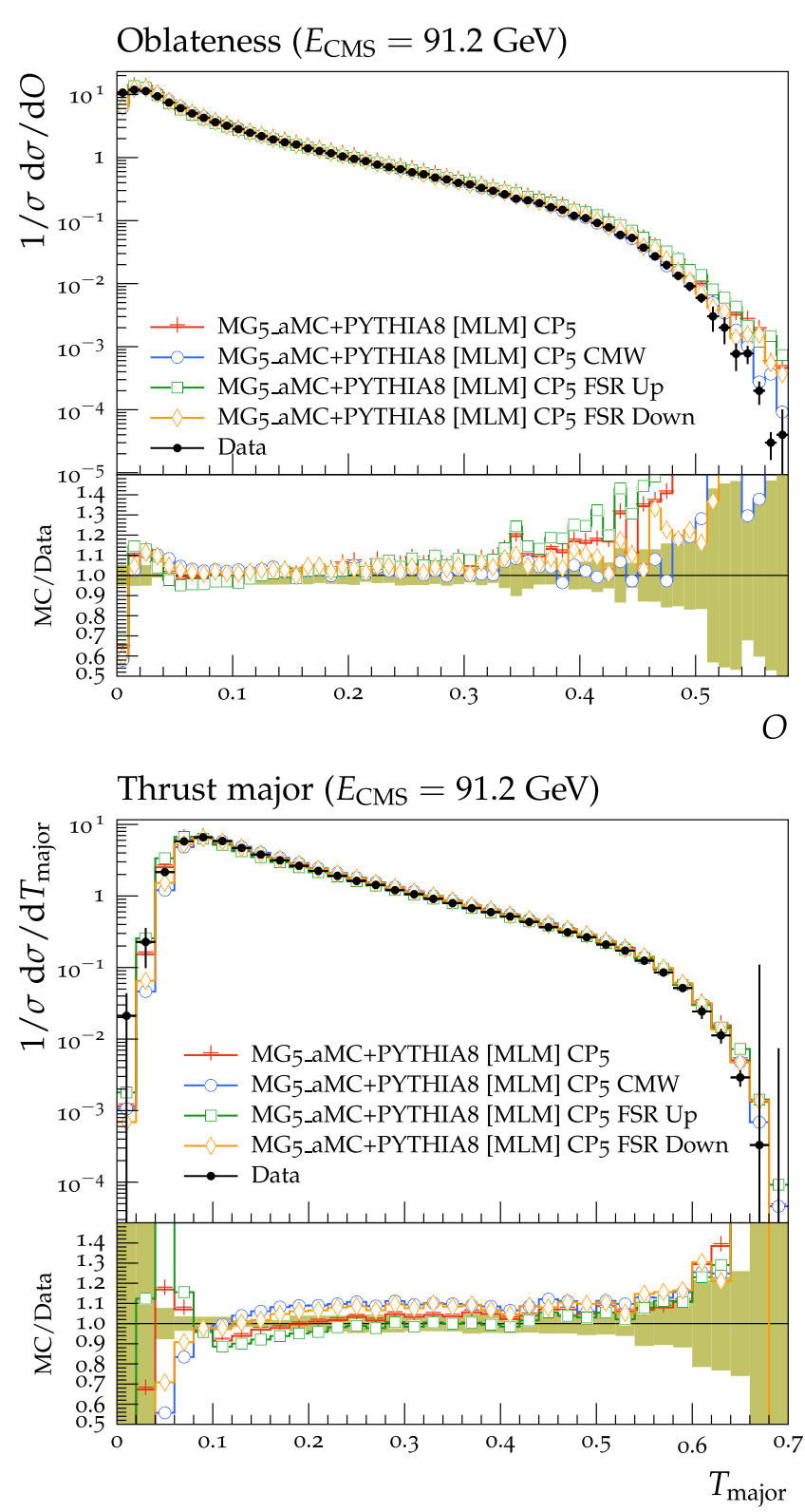

Fig. 12 The normalized cross sections as a function of event-shape variables, oblateness (upper left), sphericity (upper right), thrust (lower left), and thrust major (lower right) from the ALEPH $\sqrt{s}=91.2 \mathrm{GeV}$ analysis [45], compared with the predictions by MG5_aMC + PYTHIA8 with $k_{\mathrm{T}}-\mathrm{MLM}$ merging, for tune CP5, CP5 with CMW rescaling, CP5 FSR up, and CP5 FSR down. The ratio of the simulations to the data

nents. A better description might be provided by tunes using the Donnachie-Landshoff [51] or minimum-bias Rockefeller [52] diffractive models.

\subsection{Comparisons using observables in multijet final states}

In this subsection, we present comparisons of observables measured in multijet final states. For these studies, the
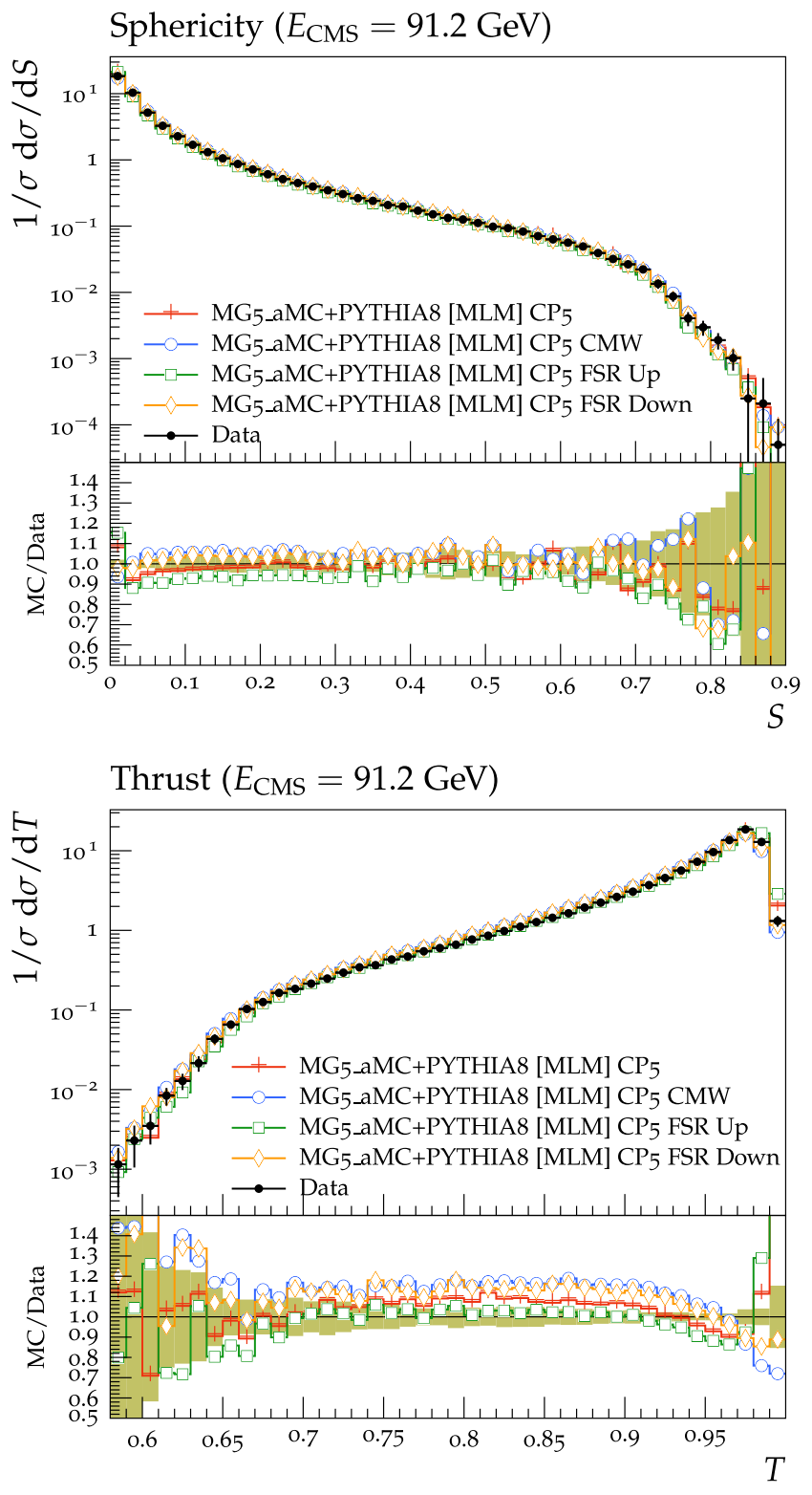

(MC/Data) is also shown, where the shaded band indicates the total experimental uncertainty in the data. Vertical lines drawn on the data points refer to the total uncertainty in the data. Vertical lines drawn on the MC points refer to the statistical uncertainty in the predictions. Horizontal bars indicate the associated bin width

NLO dijet MEs implemented in the POWHEG event generator merged with the PYTHIA8 simulation of the PS and $\mathrm{UE}$ are used. The merging between the POWHEG ME calculations and the PYTHIA8 UE simulation is performed using the shower-veto procedure, which rejects showers if their transverse momentum is greater than the minimal $p_{\mathrm{T}}$ of all final-state partons simulated in the ME (parameter $p_{\mathrm{T}}^{\text {hard }}=2 \mathrm{GeV}$ [53]). Variables in multijet events, such as 


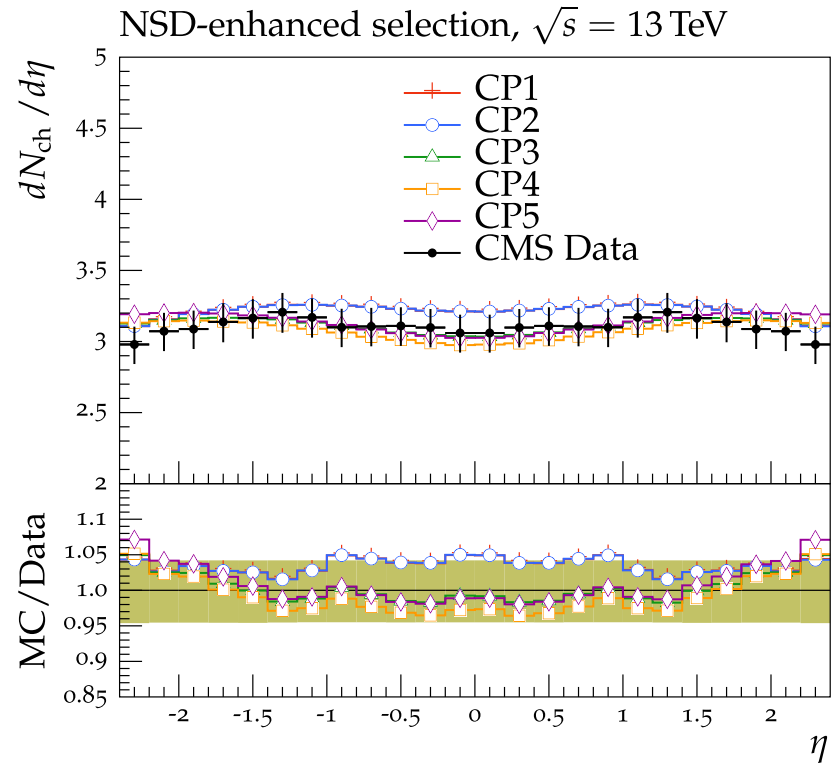

Fig. 13 The pseudorapidity distribution $\left(p_{\mathrm{T}}>0.5 \mathrm{GeV},|\eta|<2.4\right)$ for the NSD-enhanced (left) and the SD-enhanced (right) event selection of charged particles in inelastic proton-proton collisions, from the CMS $\sqrt{s}=13 \mathrm{TeV}$ analysis [24]. The data are compared with the CMS PYTHIA8 LO-PDF tunes CP1 and CP2, the CMS PYTHIA8 NLO-PDF tune $\mathrm{CP} 3$, and the CMS PYTHIA8 NNLO-PDF tunes CP4 and CP5. The

jet transverse momenta or azimuthal dijet correlations, are expected to be less affected by MPI contributions, since jets at high $p_{\mathrm{T}}(>100 \mathrm{GeV})$ mainly originate from the hard scattering or additional hard emissions, which are simulated in the POWHEG calculation by the ME formalism. However, the MPI contribution still has some impact because it adds an average energy offset to the event, which is then included in the jet reconstruction $[54,55]$. The predictions reproduce well inclusive jet cross sections as a function of jet $p_{\mathrm{T}}$ at both central and forward jet rapidities, irrespective of the cone size (0.4 or 0.7) used for the jet clustering algorithm [56].

Figure 17 shows the normalized cross section [57] as a function of the azimuthal difference $\Delta \phi_{1,2}$ between the two leading jets for two different selections on the leading jet $p_{\mathrm{T}}: 200<p_{\mathrm{T}}<300 \mathrm{GeV}$ and $300<p_{\mathrm{T}}<400 \mathrm{GeV}$. The results indicate that UE tunes based on an NLO evaluation of $\alpha_{S}\left(m_{\mathrm{Z}}\right)$ describe the data better than UE tunes based on LO evolution. In particular, the better agreement is driven by the lower value of $\alpha_{S}^{\mathrm{ISR}}\left(m_{\mathrm{Z}}\right)$. In fact, predictions obtained with POWHEG merged with PYTHIA8 with the CUETP8M1 or $\mathrm{CP} 2$ tune exhibit a strong jet decorrelation, due to a large contribution from emissions simulated from the PS, and they overestimate the cross sections at small and medium $\Delta \phi_{1,2}$ values $\left(\Delta \phi_{1,2}<2.4\right)$. The PS component is reduced by the lower value of $\alpha_{S}\left(m_{\mathrm{Z}}\right)$, which increases the degree of correlation between the selected jets, resulting in a better description of the data by predictions of the $\mathrm{CP} 4$ and $\mathrm{CP} 5$ tunes. A similar

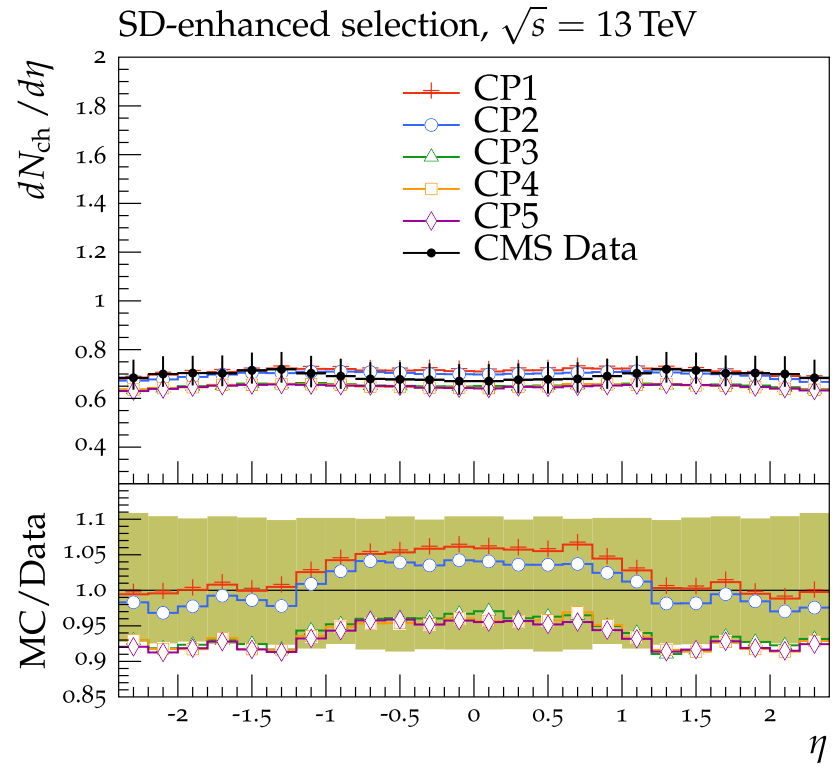

ratio of the simulations to the data (MC/Data) is also shown, where the shaded band indicates the total experimental uncertainty in the data. Vertical lines drawn on the data points refer to the total uncertainty in the data. Vertical lines drawn on the MC points refer to the statistical uncertainty in the predictions. Horizontal bars indicate the associated bin width

outcome was also observed for an analogous measurement performed at the D0 experiment at $\sqrt{s}=1.96 \mathrm{TeV}$ [60]. In general, predictions obtained from POWHEG + PYTHIA8 tend to differ from the data at low and intermediate $\Delta \phi_{1,2}$ values $\left(\Delta \phi_{1,2}<2.7\right)$ by $10-40 \%$.

\subsection{Comparisons using observables sensitive to double-parton scattering}

In this subsection, we present comparisons of predictions of the new tunes to DPS-sensitive observables measured by the CMS experiment at $\sqrt{s}=7 \mathrm{TeV}$ in final states with four jets (4j) [61], and with two jets originating from bottom quarks (b jets) [62] and two other jets (2b2j) [63].

The topology in the transverse plane of the physics objects measured in the final state is sensitive to contributions from DPS. In particular, the $4 \mathrm{j}$ analysis performed by the CMS experiment requires two jets at high $p_{\mathrm{T}}$ (hard jets) and two jets at low $p_{\mathrm{T}}$ (soft jets); the $2 \mathrm{~b} 2 \mathrm{j}$ measurement selects two jets originating from $b$ quarks and two other jets (light-flavor jets). Both of them measured the $\Delta \mathrm{S}$ observable, defined as:

$\Delta S=\arccos \left(\frac{\vec{p}_{\mathrm{T}, 1} \cdot \vec{p}_{\mathrm{T}, 2}}{\left|\vec{p}_{\mathrm{T}, 1}\right|\left|\vec{p}_{\mathrm{T}, 2}\right|}\right)$,

where $\vec{p}_{\mathrm{T}, 1}$ refers to the momentum of the hard-jet or bottom jet pair system and $\vec{p}_{\mathrm{T}, 2}$ to that of the soft-jet or light-flavor 

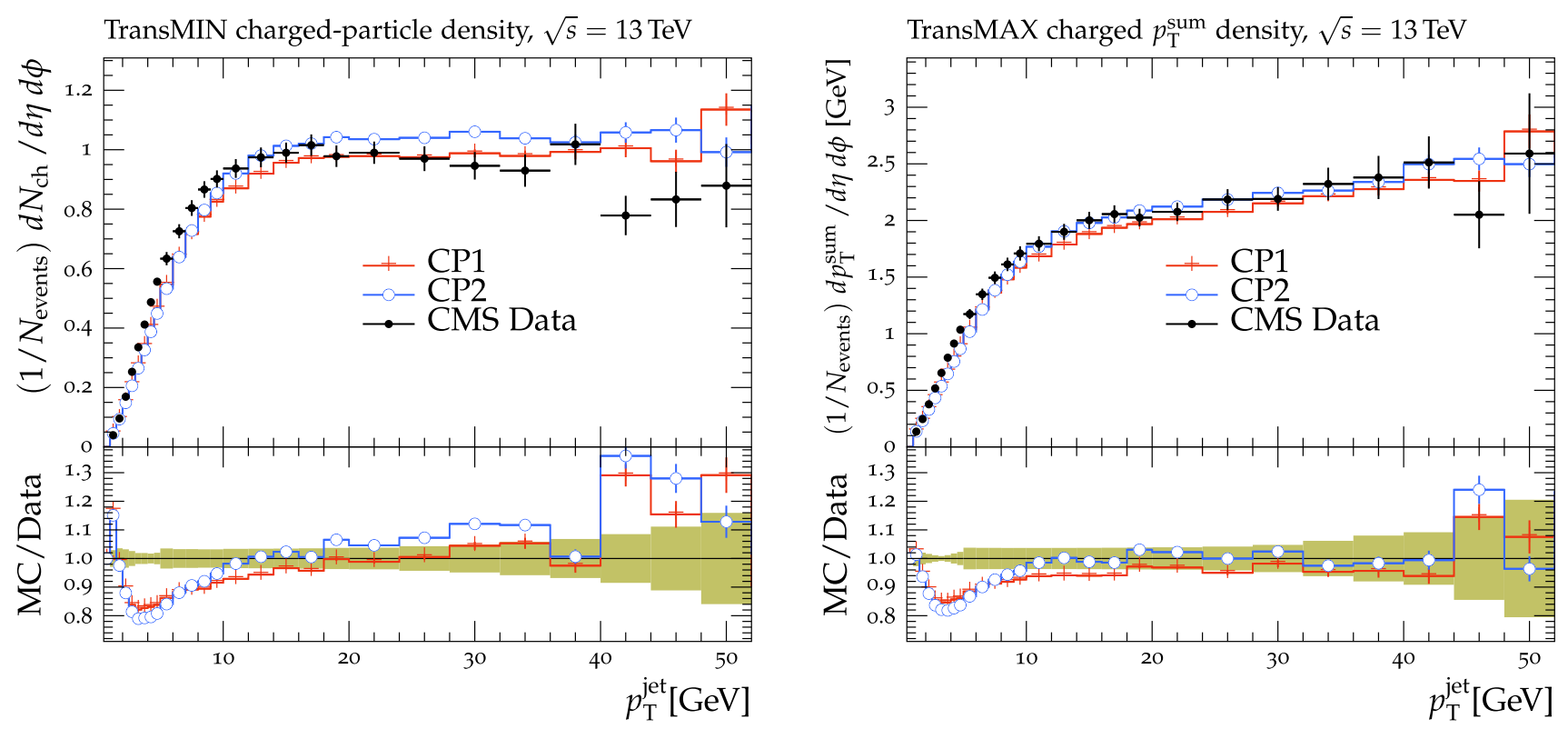

TransMIN charged-particle density, $\sqrt{s}=13 \mathrm{TeV}$
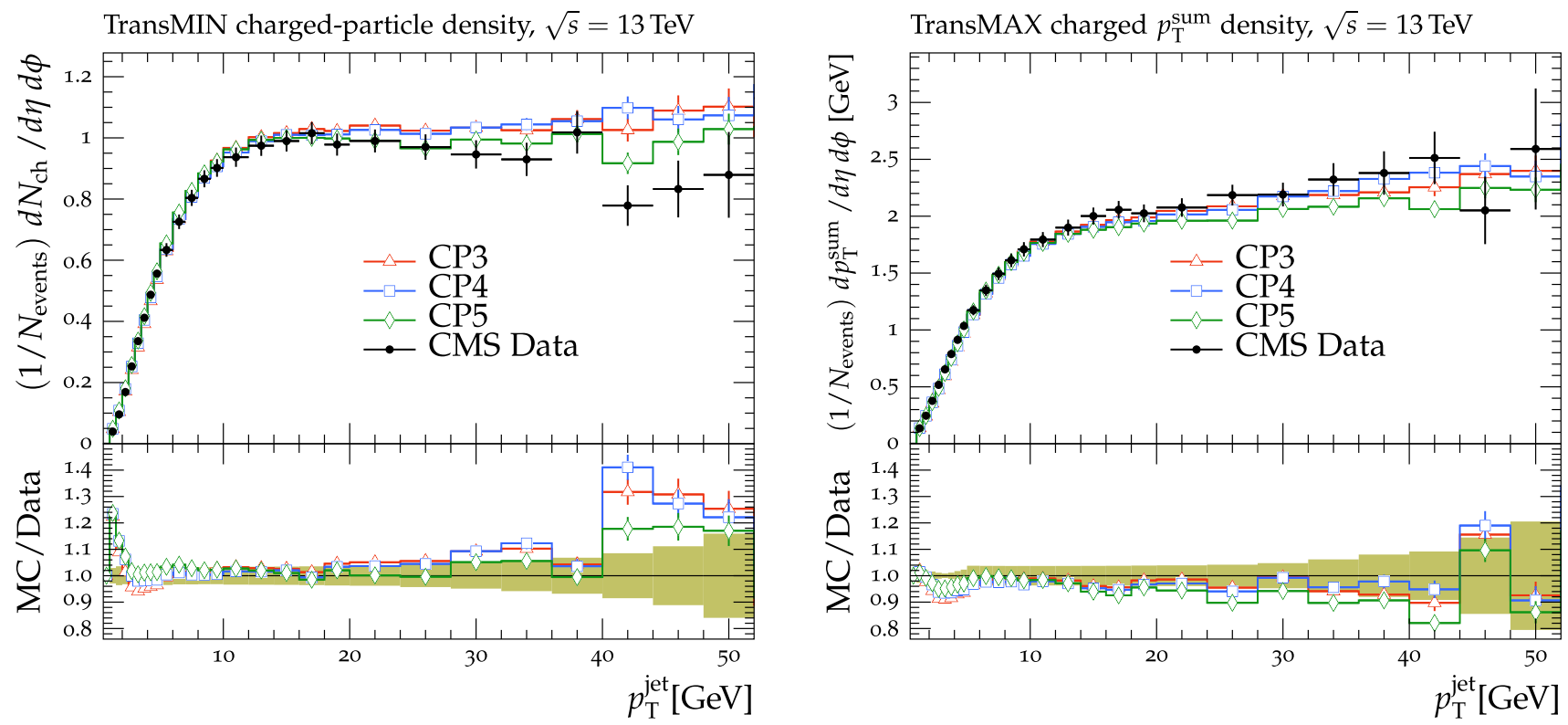

Fig. 14 The transMIN charged-particle multiplicity (left column) and $p_{\mathrm{T}}$ sum densities (right column) for particles with $p_{\mathrm{T}}>0.5 \mathrm{GeV}$ in $|\eta|<2.0$ as a function of the transverse momentum of the leading charged-particle jet, $p_{\mathrm{T}}^{\text {jet }}$, from the CMS $\sqrt{s}=13 \mathrm{TeV}$ analysis [16]. The upper-row plots show the LO tunes, while the lower-row plots show the higher-order tunes. The ratio of the simulations to the data

(MC/Data) is also shown, where the shaded band indicates the total experimental uncertainty in the data. Vertical lines drawn on the data points refer to the total uncertainty in the data. Vertical lines drawn on the MC points refer to the statistical uncertainty in the predictions. Horizontal bars indicate the associated bin width

jet pair system. This variable relates the production planes of the hard (bottom) jet and soft- (light-flavor) jet pairs. Details of the event selection and of the specific analyses can be found in Refs. [61] and [63].

Assuming that the two hard scatterings occurring within the same collision are completely independent of each other, the DPS cross section for a given process can be expressed through the inclusive partonic cross sections of the two single

scatterings and an effective cross section, $\sigma_{\text {eff. In a geomet- }}$ rical approach, this cross section is related to the transverse size of the proton and to the total inelastic proton-proton (pp) cross section $[64,65]$. When no correlations among the partons inside the proton are present, $\sigma_{\text {eff }}$ is similar to the inelastic pp cross section. In this simple factorized approach, one expects $\sigma_{\text {eff }}$ to be independent of the partonic final states of the two hard processes occurring within the same collision. 
Energy flow, inclusive selection, $\sqrt{s}=13 \mathrm{TeV}$

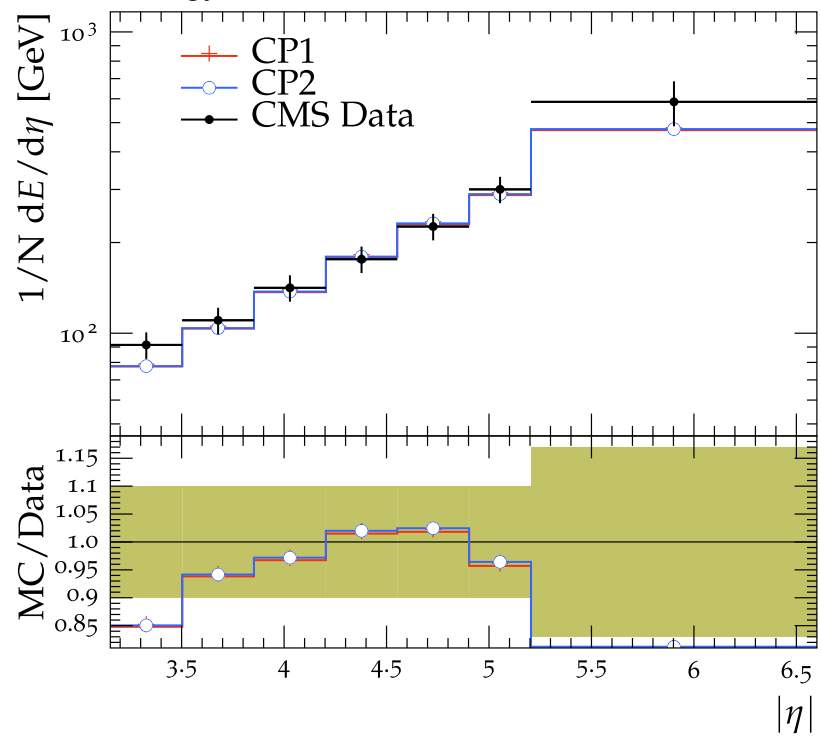

Fig. 15 The energy flow measured in an inclusive selection as a function of pseudorapidity, from the CMS $\sqrt{s}=13 \mathrm{TeV}$ analysis [47]. The data are compared with the CMS PYTHIA8 LO-PDF tunes CP1 and CP2 (left), and with the CMS PYTHIA8 NLO-PDF tune CP3 and the CMS PYTHIA8 NNLO-PDF tunes CP4 and CP5 (right). The ratio of the sim-
Energy flow, inclusive selection, $\sqrt{s}=13 \mathrm{TeV}$

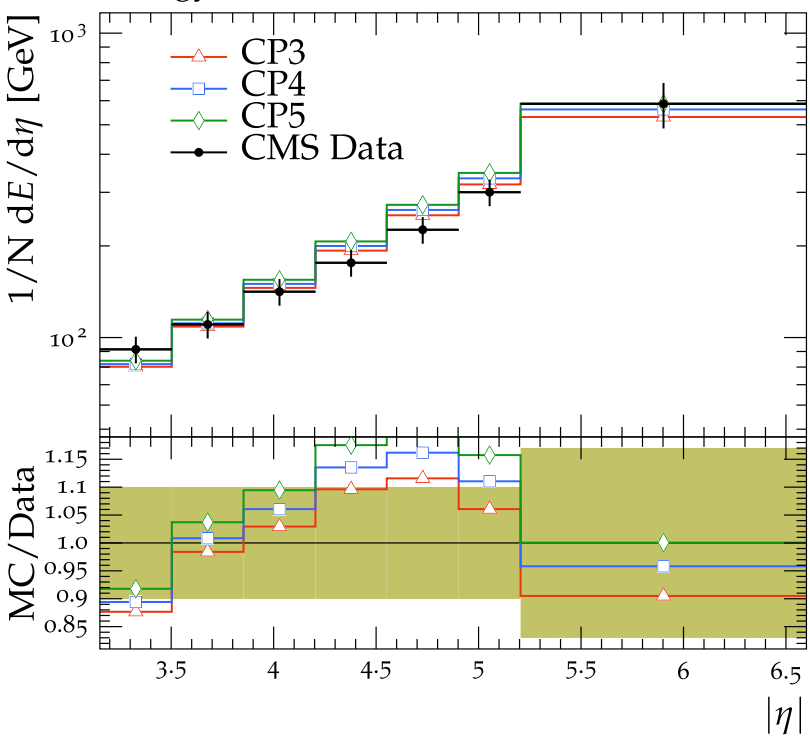

ulations to the data (MC/Data) is also shown, where the shaded band indicates the total experimental uncertainty in the data. Vertical lines drawn on the data points refer to the total uncertainty in the data. Vertical lines drawn on the MC points refer to the statistical uncertainty in the predictions. Horizontal bars indicate the associated bin width

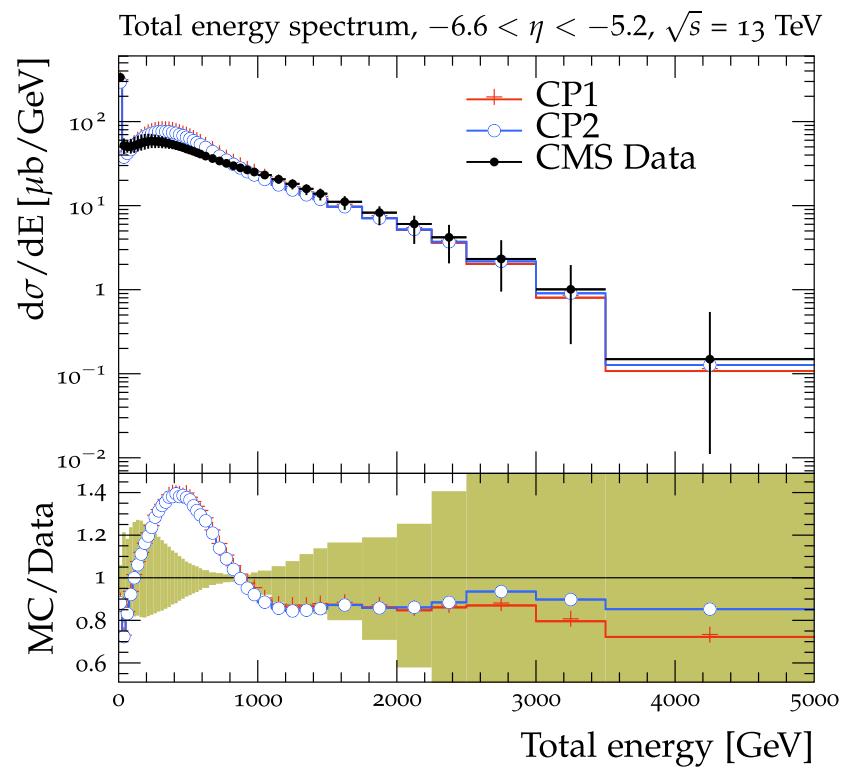

Fig. 16 The total energy spectrum measured in the pseudorapidity interval $-6.6<\eta<-5.2$, from the CMS $\sqrt{s}=13 \mathrm{TeV}$ analysis [49]. The data are compared with the CMS PYTHIA8 LO-PDF tunes CP1 and CP2 (left), and with the CMS PYTHIA8 NLO-PDF tune CP3 and the CMS PYTHIA8 NNLO-PDF tunes CP4 and CP5 (right). The ratio of the simulations to the data (MC/Data) is also shown, where the

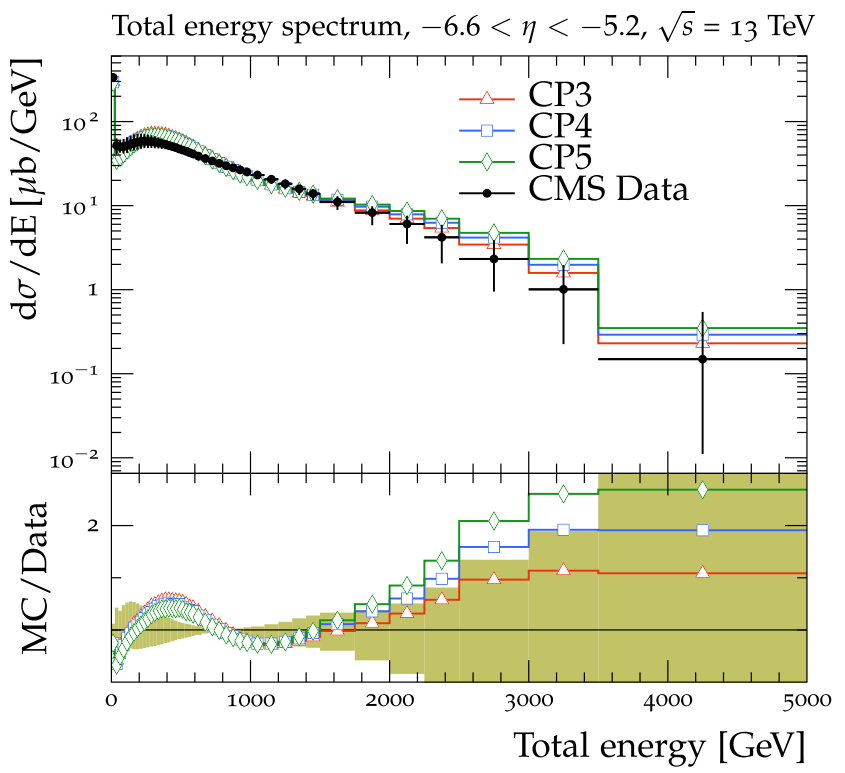

shaded band indicates the total experimental uncertainty in the data. Vertical lines drawn on the data points refer to the total uncertainty in the data. Vertical lines drawn on the MC points refer to the statistical uncertainty in the predictions. Horizontal bars indicate the associated bin width 


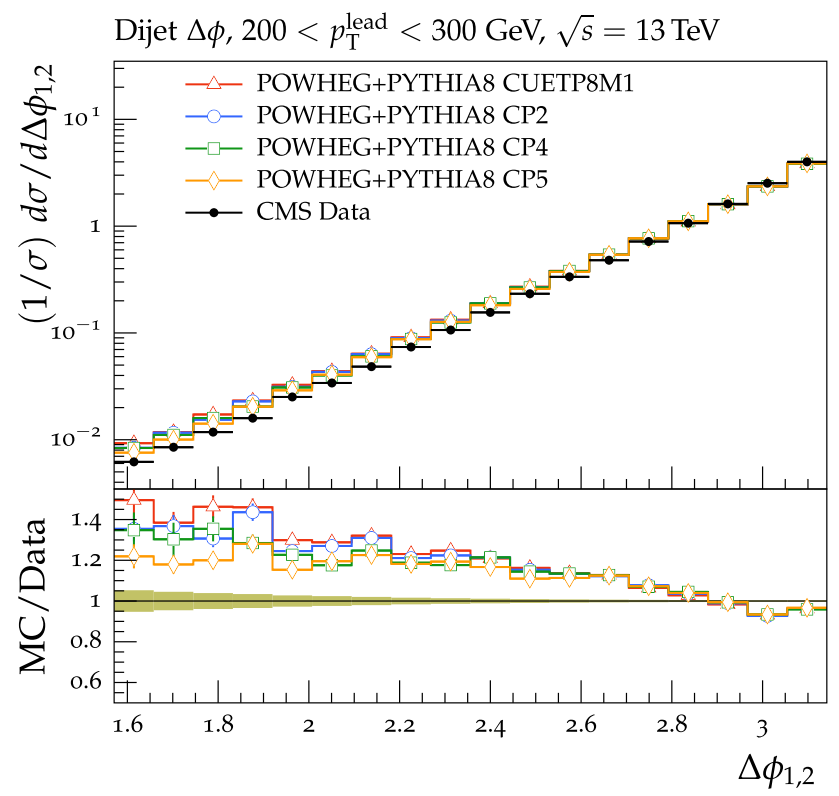

Fig. 17 The azimuthal difference $\Delta \phi_{1,2}$ between the leading two jets with $|\eta|<2.4$ in dijet events with leading-jet transverse momentum in the range (left) $200<p_{\mathrm{T}}^{\text {lead }}<300 \mathrm{GeV}$ and (right) $300<p_{\mathrm{T}}^{\text {lead }}<$ $400 \mathrm{GeV}$, from the CMS $\sqrt{s}=13 \mathrm{TeV}$ analysis [57]. The jets are reconstructed using the anti- $k_{\mathrm{T}}$ jet finding algorithm $[58,59]$ with a distance parameter of 0.4. The data are compared with predictions of the NLO dijet ME calculation from POWHEG, interfaced to the PYTHIA8 tunes CUETP8M1, CP2, CP4, and CP5. Tunes CP1 and CP3 are not shown

In PYTHIA8, the value of $\sigma_{\text {eff }}$ is calculated by dividing the ND cross section by the so-called "enhancement factor", which depends on the parameters of the overlap matter distribution function and on pTORef [40]. For central pp collisions, the enhancement factor tends to be large, translating to a lower value of $\sigma_{\text {eff }}$ and a larger DPS contribution. For peripheral interactions, enhancement factors are small, giving large values of $\sigma_{\text {eff }}$ and a small DPS contribution.

Table 4 shows the values of $\sigma_{\text {eff }}$ published by the CMS Collaboration for the $4 \mathrm{j}$ and the $2 \mathrm{~b} 2 \mathrm{j}$ measurements. A previous study [5] concluded that observables sensitive to semihard MPI and those sensitive to DPS cannot be described by a single set of parameters. Table 5 displays the $\sigma_{\text {eff }}$ values obtained from the new CMS UE tunes. The central values of $\sigma_{\text {eff }}$ are consistent among the new tunes and are slightly larger than the values of the DPS-based tunes [5].

Figure 18 shows the comparisons of predictions obtained from PYTHIA8 with tunes CUETP8M1, CP2, CP4, and CP5 to the DPS observables measured in the $4 j$ and $2 b 2 j$ final states. Predictions from the CP2 tune based on a LO PDF set describe the central values better than the $\mathrm{CP} 4$ and $\mathrm{CP} 5$ tunes based on an NNLO PDF set or the old tune CUETP8M1. This is due to the different pTORef value used by CP2, CP4, and CP5, which determines the amount of simulated MPI. The value of the pTORef parameter is driven by the distribution

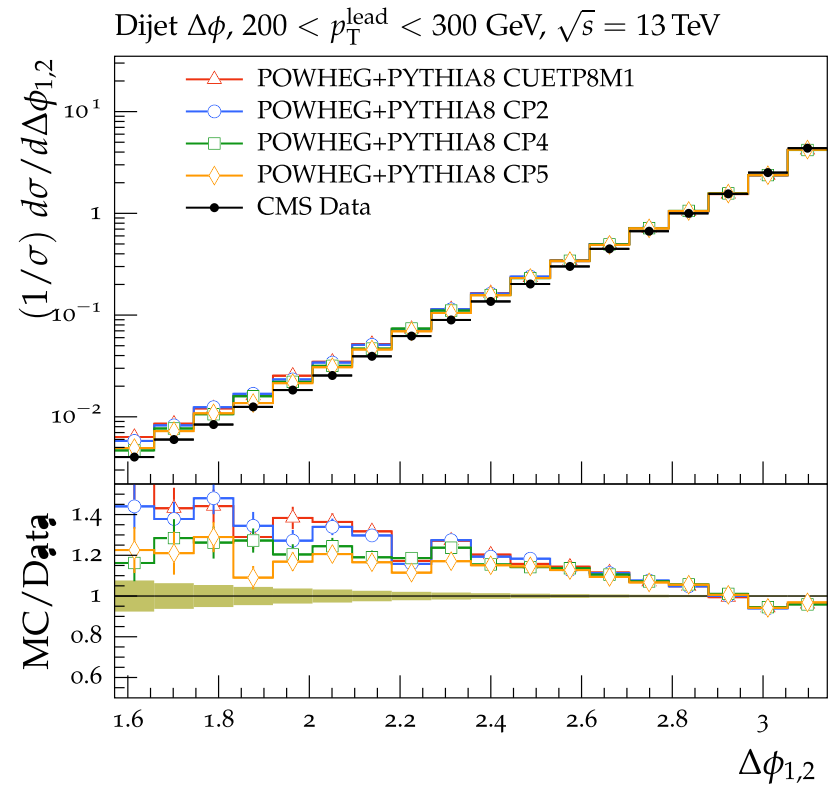

in the plot but present a similar behavior as tunes CP2 and CP4. The ratios of simulations to the data (MC/Data) are also shown, where the shaded band indicates the total experimental uncertainty in the data. Vertical lines drawn on the data points refer to the total uncertainty in the data. Vertical lines drawn on the MC points refer to the statistical uncertainty in the predictions. Horizontal bars indicate the associated bin width

Table 4 Values of $\sigma_{\text {eff }}$ at $\sqrt{s}=7 \mathrm{TeV}$ published by the CMS Collaboration for the four-jet final states, obtained by fitting predictions of the PYTHIA8 MC event generator to DPS-sensitive measured observables

\begin{tabular}{lll}
\hline Final state & Generator & $\sigma_{\text {eff }}(\mathrm{mb})(\sqrt{s}=7 \mathrm{TeV})$ \\
\hline $4 \mathrm{j}$ & PYTHIA8 & $19.0_{-3.0}^{+4.7}[5]$ \\
$2 \mathrm{~b} 2 \mathrm{j}$ & PYTHIA8 & $23.2_{-2.5}^{+3.3}[67]$ \\
\hline
\end{tabular}

of the gluon distribution function at low $x$, which is very different in LO and NNLO PDF sets. Additionally, predictions obtained with CP4 describe the DPS-sensitive observables better than CP5. This is due to the different rapidity ordering used for the PS emissions in the two tunes. By removing the rapidity ordering for the PS emissions (CP4), the simulation produces more radiation and decreases the correlation between the selected jet pairs compared to CP5. This reduced jet correlation tends to mimic a DPS event by producing low values of $\Delta \mathrm{S}$. We have checked that the observables sensitive to color coherence, which were measured by the CMS experiment at $\sqrt{s}=7 \mathrm{TeV}$ [66], are well described by predictions from both $\mathrm{CP} 4$ and $\mathrm{CP} 5$ tunes, despite the difference in the rapidity ordering of the PS simulation between the two tunes. 
Table 5 Values of $\sigma_{\text {eff }}$ at $\sqrt{s}=7$ and $13 \mathrm{TeV}$ obtained with the new CMS UE tunes

\begin{tabular}{lll}
\hline & $\sqrt{s}=7 \mathrm{TeV}$ & $\sqrt{s}=13 \mathrm{TeV}$ \\
$\sigma_{\text {eff }}(\mathrm{mb})$ & $\sigma_{\text {eff }}(\mathrm{mb})$ \\
\hline $\mathrm{CP} 1$ & $26.3_{-1.7}^{+1.0}$ & $27.8_{-1.4}^{+1.1}$ \\
$\mathrm{CP} 2$ & $24.7_{-1.6}^{+1.0}$ & $26.0_{-1.3}^{+1.0}$ \\
$\mathrm{CP} 3$ & $24.1_{-1.5}^{+1.0}$ & $25.2_{-1.3}^{+1.0}$ \\
$\mathrm{CP} 4$ & $23.9_{-1.5}^{+1.0}$ & $25.3_{-1.4}^{+1.1}$ \\
$\mathrm{CP5}$ & $24.0_{-1.6}^{+1.0}$ & $25.3_{-1.3}^{+1.0}$ \\
\hline
\end{tabular}

6.5 Comparisons using observables in top quark production

In the following, we investigate how the new PYTHIA8 tunes describe the CMS $\mathrm{t} \overline{\mathrm{t}}$ data when different ME generators, namely POWHEG and MG5_aMC, are employed. Both ME configurations use the NNPDF3.1 NNLO PDF with $\alpha_{S}\left(m_{\mathrm{Z}}\right)=$ 0.118 and assume a top quark mass $\left(m_{\mathrm{t}}\right)$ value of $172.5 \mathrm{GeV}$.

In the POWHEG configuration, the ME heavy quark production mode $[36,39,68]$ is used. In this configuration, POWHEG simulates inclusive $t \bar{t}$ production at NLO, where the first additional jet is computed at LO, while MG5_aMC performs the calculation with up to 2 additional jets at NLO, with a third jet simulated at LO. The POWHEG generator scales the real emission cross section by a damping function that controls the ME-PS merging and that regulates the high- $p_{\mathrm{T}}$ radiation.

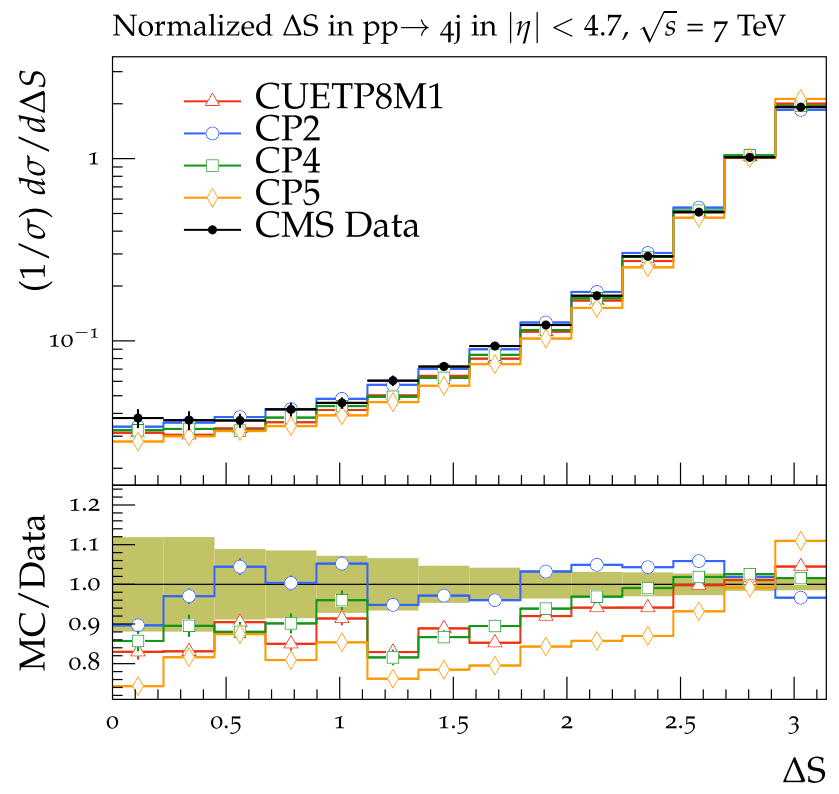

Fig. 18 The correlation observable $\Delta \mathrm{S}$ measured in $4 \mathrm{j}$ (left) and $2 \mathrm{~b} 2 \mathrm{j}$ (right) production, compared to predictions of PYTHIA 8 tunes CUETP8M1, CP2, CP4, and CP5, from the CMS $\sqrt{s}=7 \mathrm{TeV}$ analyses $[61,63]$. Tunes CP1 and CP3 are not shown in the plot but show a similar behaviour as, respectively, tunes $\mathrm{CP} 2$ and $\mathrm{CP} 4$. The ratios of
The damping variable used in the POWHEG simulation is set to 1.379 times $m_{\mathrm{t}}$, a value derived from data at $\sqrt{s}=8 \mathrm{TeV}$ in the dilepton channel using a similar ME calculation and assuming the CP5 tune. The factorization and renormalization scales are assumed equal to the transverse mass of the top quark, $m_{\mathrm{T}}^{\mathrm{t}}=\sqrt{m_{\mathrm{t}}^{2}+p_{\mathrm{T}}^{2}}$. The minimum $p_{\mathrm{T}}$ for the emission of light quarks in POWHEG is $0.8 \mathrm{GeV}$. The pThard parameter is set to 0 and the POWHEG hardness criterion, defined by the pTdef option, is set to 1 . The merging scale in MG5_aMC is set to $40 \mathrm{GeV}$, and the threshold applied to regulate multijet MEs in the MG5_aMC FxFx merging procedure, is $20 \mathrm{GeV}$.

Distributions [69] in the lepton+jets channel are compared to predictions from different tunes using various settings, namely, POWHEG + PYTHIA8, and MG5_aMC+PYTHIA8 with FxFx merging [38], referred to as MG5_aMC [FxFx] hereafter, with the CUETP8M1, CP2, CP4, and CP5 tunes. Figure 19 (upper panel) displays the normalized t $\bar{t}$ cross section in bins of $p_{\mathrm{T}}$ of the top quark decaying leptonically $\left(\mathrm{t}_{\ell}\right)$, in data and simulation. For all tunes, POWHEG + PYTHIA8 predictions have deviations below $10 \%$ with respect to the central values of the data. The central values of predictions from MG5_aMC $[\mathrm{FxFx}]$ and data agree within $\approx 10 \%$ for $p_{\mathrm{T}}\left(\mathrm{t}_{\ell}\right)<400 \mathrm{GeV}$ and within $\approx 20 \%$ for higher $p_{\mathrm{T}}$.

Figure 19 (middle panel) shows the normalized $t \bar{t}$ cross section in bins of $m(\mathrm{t} \overline{\mathrm{t}})$ in data and simulation. Predictions from POWHEG and MG5_aMC [FxFx] with the new tunes

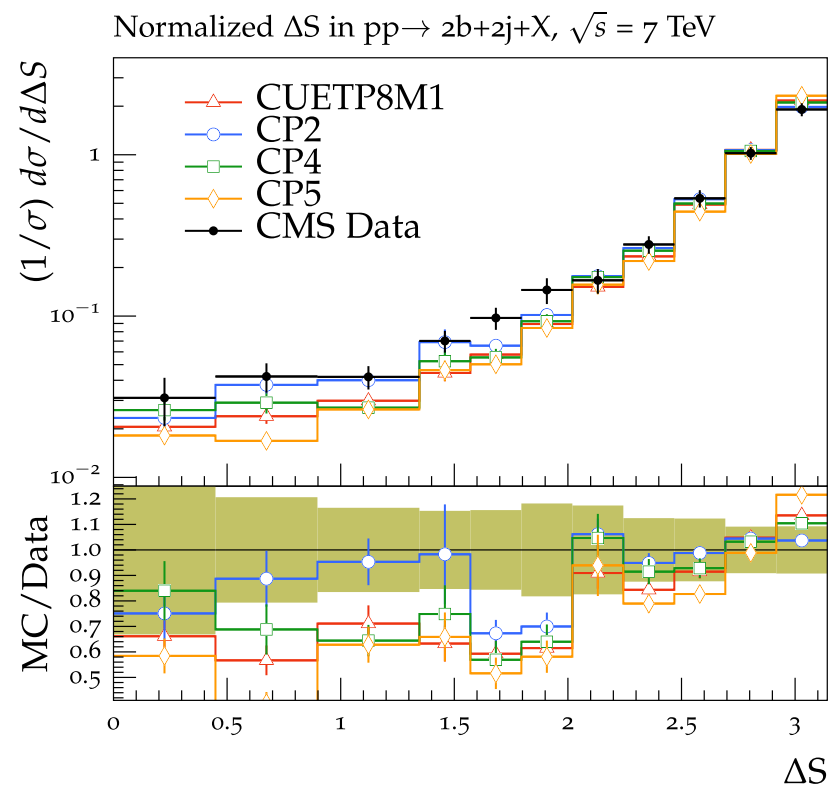

simulations to the data (MC/Data) are also shown, where the shaded band indicates the total experimental uncertainty in the data. Vertical lines drawn on the data points refer to the total uncertainty in the data. Vertical lines drawn on the MC points refer to the statistical uncertainty in the predictions. Horizontal bars indicate the associated bin width 

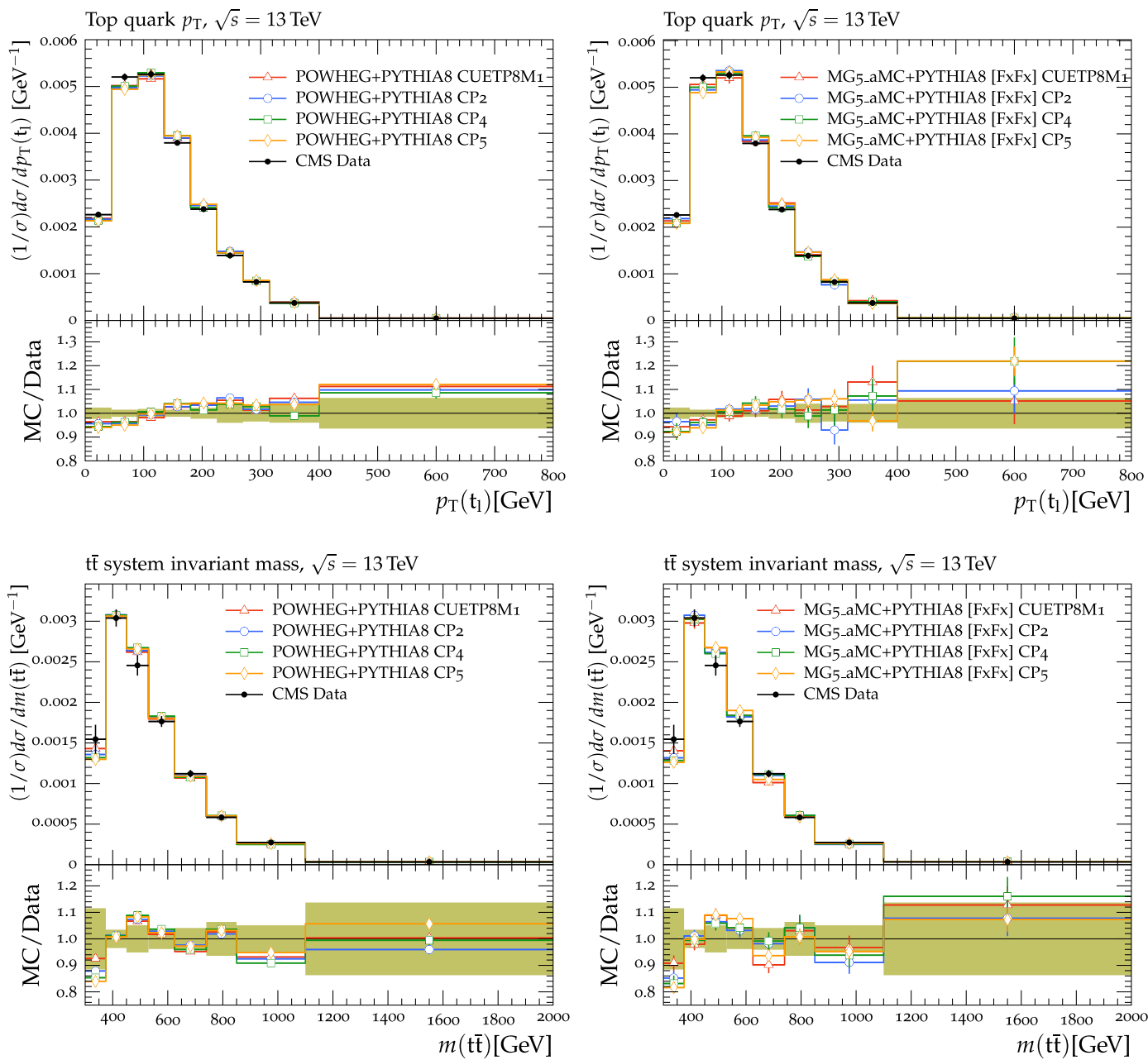

Jet multiplicity in $\mathrm{t} \overline{\mathrm{t}}$ events, $\sqrt{\mathrm{s}}=13 \mathrm{TeV}$

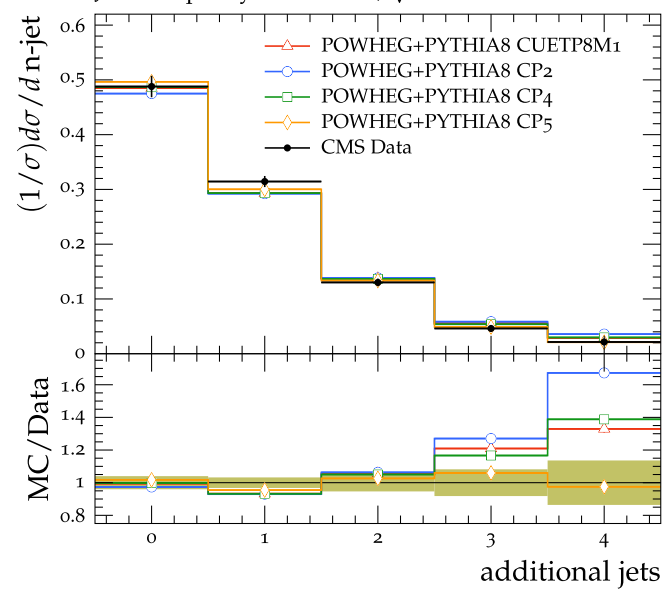

Fig. 19 The normalized $t \bar{t}$ cross section in the lepton+jets channel, as a function of the transverse momentum of the top quark for leptonically decaying top quarks $\left(t_{\ell}\right)$ (upper), the invariant mass of the $t \bar{t}$ system, $m(\mathrm{t} \overline{\mathrm{t}})$ (middle), and in bins of number of additional jets (lower) from CMS $\sqrt{s}=13 \mathrm{TeV}$ analysis [69]. The data are compared with the predictions of POWHEG (left) and MG5_aMC [FxFx] (right). In both cases, the PS simulation is done with the PYTHIA8 tunes CUETP8M1, CP2,

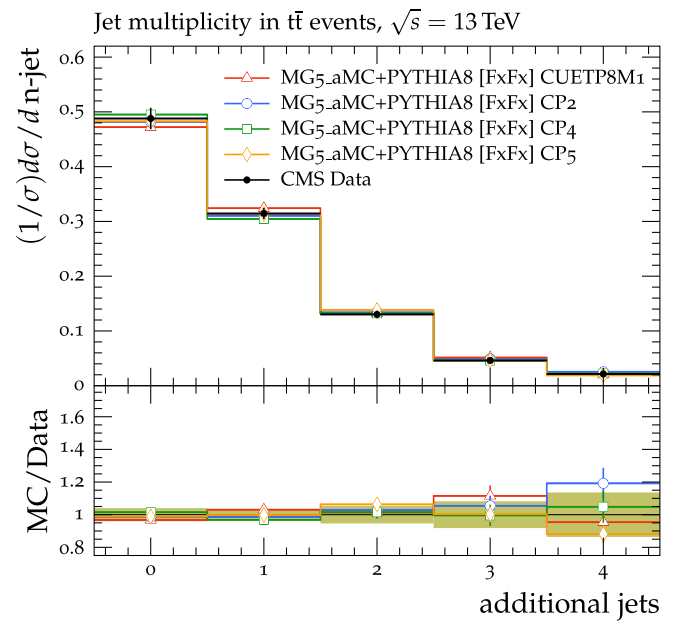

$\mathrm{CP} 4$, or CP5. Tunes CP1 and CP3 are not shown in the plot but present a similar behaviour as, respectively, tunes $\mathrm{CP} 2$ and $\mathrm{CP} 4$. The ratios of simulations to the data (MC/Data) are also shown, where the shaded band indicates the total experimental uncertainty in the data. Vertical lines drawn on the data points refer to the total uncertainty in the data. Vertical lines drawn on the MC points refer to the statistical uncertainty in the predictions. Horizontal bars indicate the associated bin width 


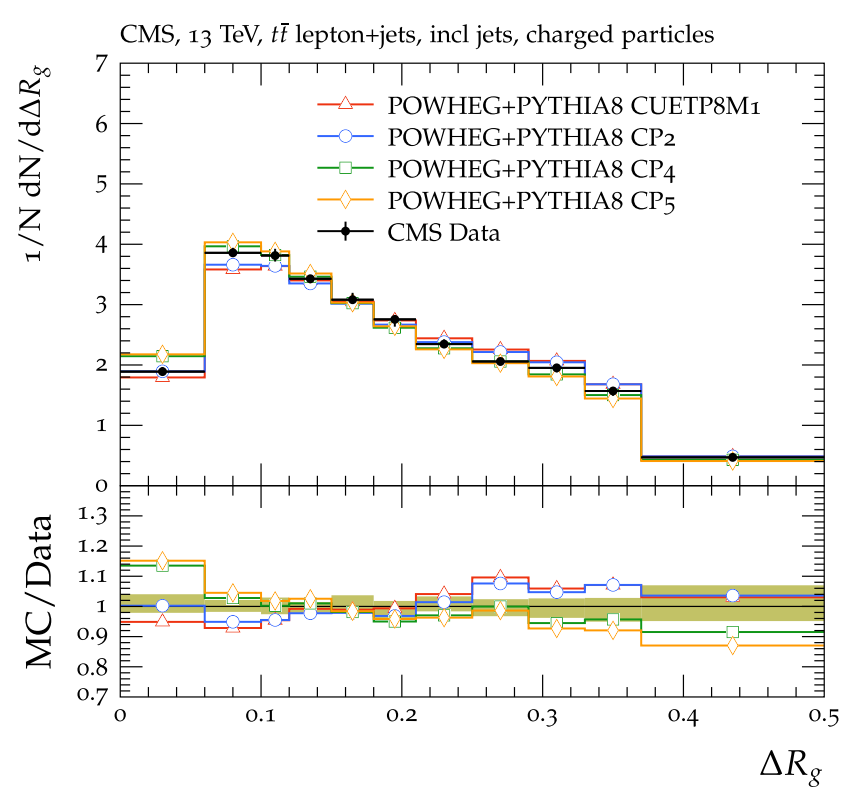

Fig. 20 Comparison with the measurement [70] of the angle between two groomed subjets, $\Delta R_{g}$ in t $\bar{t}$ events predicted by POWHEG + PYTHIA 8 for the different tunes. The data are compared to tunes CUETP8M1, CP2, CP4, and CP5 (left). Tunes CP1 and CP3 are not displayed but they present a similar behavior as tunes $\mathrm{CP} 2$ and $\mathrm{CP} 4$, respectively. The data are also compared to CP5, CP5 FSR up, CP5 FSR down,

describe the central values of the data reasonably well. Normalized $t \bar{t}$ cross sections in bins of number of additional jets in data and simulation in the lepton+jets channel at $\sqrt{s}=13 \mathrm{TeV}$ are shown in Fig. 19 (lower panel). The cross sections are compared with the predictions of POWHEG and of MG5_aMC $[\mathrm{FxFx}]$. The central values predicted by POWHEG + PYTHIA8 are in good agreement with data when CP5 tune is used. The value of $\alpha_{S}^{\mathrm{ISR}}\left(m_{\mathrm{Z}}\right)$ in combination with the rapidity ordering for ISR in the PYTHIA 8 simulation affects the additional jet distribution in $\mathrm{t} \overline{\mathrm{t}}$ events. Predictions obtained from POWHEG + PYTHIA8 overestimate the data when a high value of $\alpha_{S}^{\mathrm{ISR}}\left(m_{\mathrm{Z}}\right) \approx 0.13$ is used (CUETP8M1 and CP2 tunes) irrespective of rapidity ordering for ISR. It is observed that even when $\alpha_{S}^{\mathrm{ISR}}\left(m_{\mathrm{Z}}\right)=$ 0.118 is used, predictions from the CP4 tune overshoot the data at high jet multiplicities. A much better agreement of central values is obtained only when rapidity ordering for ISR is switched on in the PYTHIA8 simulation and $\alpha_{S}^{\mathrm{ISR}}\left(m_{\mathrm{Z}}\right)=0.118$ is used as in the CP5 tune. Predictions from MG5_aMC [FxFx]+PYTHIA8 with CUETP8M1, CP2, $\mathrm{CP} 4$, and CP5 tunes describe the central values of the data reasonably well.

Comparisons are also made using jet substructure observables in tit events in the lepton+jets channel using measurements by CMS at $\sqrt{s}=13 \mathrm{TeV}$ [70]. Figure 20 displays the comparisons using the distribution of the angle between two groomed subjets, $\Delta R_{g}$, which is found to be the most

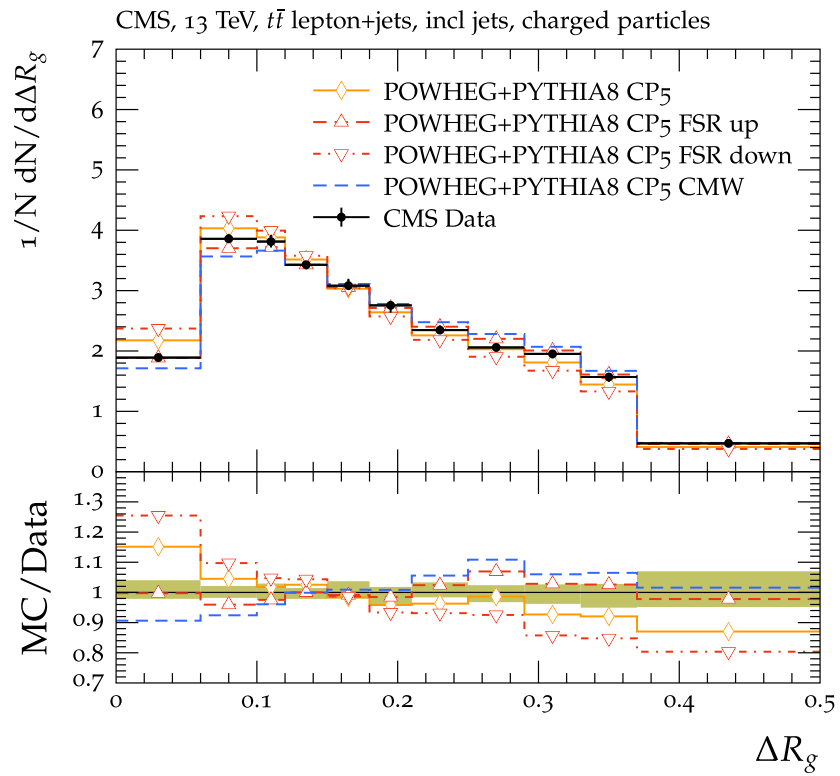

and CP5 with CMW rescaling (right). The ratios of simulations to the data (MC/Data) are also shown, where the shaded band indicates the total experimental uncertainty in data. Vertical lines drawn on the data points refer to the total uncertainty in the data. Horizontal bars indicate the associated bin width

sensitive to $\alpha_{S}^{\mathrm{FSR}}\left(m_{\mathrm{Z}}\right)$ [70]. The data are compared to simulations with the tunes CUETP8M1, CP2, CP4, and CP5, as well as CP5 FSR up $\left(\alpha_{S}^{\mathrm{FSR}}\left(m_{\mathrm{Z}}\right)=0.122\right)$, CP5 FSR down $\left(\alpha_{S}^{\mathrm{FSR}}\left(m_{\mathrm{Z}}\right)=0.115\right)$, and CP5 with CMW rescaling. It is observed that tunes with higher $\alpha_{S}^{\mathrm{FSR}}\left(m_{\mathrm{Z}}\right)$ (CUETP8M1, CP2, and CP5 FSR up) describe the data better. Tune CP5 with CMW rescaling resolves the discrepancy of CP5 at high $\Delta R_{g}$, but worsens the description at $\Delta R_{g} \sim 0.27$ compared to CP5. It should be noted that a fit to the $\Delta R_{g}$ distribution using a b-enriched sample yields $\alpha_{S}^{\mathrm{FSR}}\left(m_{\mathrm{Z}}\right)=0.130_{-0.020}^{+0.016}$ [70] without CMW rescaling, while a fit to the distirubtion of the UE observable $\overline{p_{\mathrm{T}}}$ measured in $\mathrm{t} \overline{\mathrm{t}}$ events yields

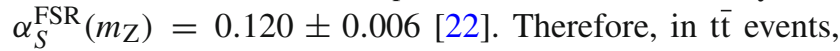
UE and jet substructure observables prefer different central $\alpha_{S}^{\mathrm{FSR}}\left(m_{\mathrm{Z}}\right)$ values, but they are compatible within uncertainties.

\subsection{Comparisons using observables in $\mathrm{W}$ and $\mathrm{Z}$ boson} production

In this subsection, we present a validation of the new CMS UE tunes for observables measured in events with a $\mathrm{W}$ or $\mathrm{Z}$ boson in the final state at $\sqrt{s}=13 \mathrm{TeV}$. For the comparisons, we use predictions obtained with MG5_aMC + PYTHIA8 at LO using the $k_{\mathrm{T}}-\mathrm{MLM}$ merging scheme, and at NLO using the FxFx merging scheme. The $k_{\mathrm{T}}-\mathrm{MLM}$ merging scale is set to $19 \mathrm{GeV}$, while for FxFx the corresponding scale is set to 
$30 \mathrm{GeV}$. In both cases the MEs include the final states with $0,1,2$, and 3 partons, and up to 2 partons are calculated at NLO precision in the FxFx case. To ease the comparison of the different tunes, the same PDF, NNPDF3.1 NNLO, and $\alpha_{S}\left(m_{\mathrm{Z}}\right)=0.118$ are used for the ME calculation independently of the tune.

First, UE observables [21] in Drell-Yan events in an invariant mass window of $81-101 \mathrm{GeV}$ around the $\mathrm{Z}$ boson peak for muonic decays are studied. The charged-particle density and transverse momentum sum are measured as a function of the $\mathrm{Z}$ boson $p_{\mathrm{T}}$ in the three regions introduced in Sect. 6.2: toward, away, and transverse. The regions are defined with respect to the $\mathrm{Z}$ boson direction. The measurements are compared with FxFx predictions obtained with the CUETP8M1, CP2, CP4, and CP5 tunes in Fig. 21. The measurements are, in general, well-described by all tunes.

The description of the cross section as a function of the jet multiplicity is also investigated in $\mathrm{Z}+$ jets [71] and $\mathrm{W}+$ jets [72] final states. The $\mathrm{Z}+$ jets measurement is restricted to the phase space where the two leptons have $p_{\mathrm{T}}>20 \mathrm{GeV}$ and $|y|<2.4$ and the dilepton mass lies in a $\pm 20 \mathrm{GeV}$ window around $91 \mathrm{GeV}$. The momenta of the photons inside a cone of $\Delta \mathrm{R}<0.1$ are added to the lepton momentum in order to partly recover the energy lost by FSR. Jets are clustered using the anti- $k_{\mathrm{T}}$ algorithm with $R=0.4$ and must satisfy the criteria $p_{\mathrm{T}}>30 \mathrm{GeV}$ and $|y|<2.4$. The distance between the selected leptons and the leading jet $\Delta R(\ell, \mathrm{j})$ must be greater than 0.4 . For the $\mathrm{W}+$ jets measurement, the phase space is restricted by a transverse mass requirement, $m_{\mathrm{T}}>$ $50 \mathrm{GeV}$, and by requirements on the muon, $p_{\mathrm{T}}>25 \mathrm{GeV}$ and $|y|<2$.4. In the $\mathrm{Z}+$ jets measurements the same clustering algorithm, the FSR recovery prescription described above, and the lepton jet separation requirement are applied.

The comparisons of the jet multiplicities to various predictions are shown in Fig. 22. The measurement of the cross section inclusive in the number of jets, $N$, is not available for the $\mathrm{W}+$ jets analysis and the lower plots start at $N=1$. The $k_{\mathrm{T}}-\mathrm{MLM}$ predictions of the jet multiplicity have little sensitivity to the UE and PS tunes, so all the tunes provide a good description of this observable, with a slightly better agreement observed for the CP2 tune. In the case of the FxFx sample, the CP5 tune predicts fewer events with a jet multiplicity of more than four with respect to the measurement. The deficit increases for increasing jet multiplicities. The CUETP8M1 tune shows a similar behaviour, though.

Predictions using the new CMS UE tunes are also compared with the $p_{\mathrm{T}}$ balance between the $\mathrm{Z}$ boson and the jets with $p_{\mathrm{T}}>30 \mathrm{GeV}$ and $|y|<2.4$ using the variable $p_{\mathrm{T}}$ bal $=\left|\vec{p}_{\mathrm{T}}(\mathrm{Z})+\sum_{\text {jets }} \vec{p}_{\mathrm{T}}\left(\mathrm{j}_{i}\right)\right|[71]$. This variable is sensitive to PS and UE. The comparison is shown in Fig. 23 for events with at least one jet. Differences between the tunes are significant only in the region below $\approx 20 \mathrm{GeV}$. The discrepancy in this region for the FxFx samples indicates that the distribution peaks at lower values for CP4 and CP5 than in data.

Results of Ref. [71] are also used to validate the description of the transverse momentum of the weak vector boson in $\mathrm{Z}+\geq 1$ jet events. The comparison is shown in Fig. 24 . The new tunes provide similar descriptions for this distribution. Predictions using $k_{\mathrm{T}}-\mathrm{MLM}$ achieve a poor agreement with the data, independently of the UE tune, with respect to FxFx, which is able to describe the transverse momentum of the $\mathrm{Z}$ boson at $p_{\mathrm{T}}>10 \mathrm{GeV}$. The region below $10 \mathrm{GeV}$ is poorly described for both FxFx and $k_{\mathrm{T}}-\mathrm{MLM}$ and the new tunes, but is well-described by predictions using the CUETP8M1 tune.

To summarize the study of weak vector boson production, the $\mathrm{CP} 2, \mathrm{CP} 4$, and $\mathrm{CP} 5$ tunes provide similar descriptions of the UE observables with a reasonable agreement with the data. In general, the CP2 tune performs better in describing variables such as $p_{\mathrm{T}}{ }^{\text {bal }}$ and $p_{\mathrm{T}}(\mathrm{Z})$. For the jet multiplicity, the CP2 and CP4 tunes are equally good in describing the measurement, whereas CP5 tends to undershoot the PS dominated region with at least five jets with a significance of 3.5 standard deviations.

\section{Summary and conclusions}

A new set of tunes for the underlying-event (UE) simulation in the PYTHIA8 event generator is obtained by fitting various measurements sensitive to soft and semihard multipartonic interactions at different collision energies. To derive these tunes, the leading order (LO), next-to-leading order (NLO), or next-to-next-to-leading order (NNLO) versions of the NNPDF3.1 parton distribution function (PDF) set for the simulation of the underlying-event components are used. In these tunes, the values of the strong coupling, $\alpha_{S}\left(m_{\mathrm{Z}}\right)$, used for the simulation of hard scattering, initial- and final-state radiation, and multiple-parton interactions are chosen consistent with the order of the PDF used. In the LO NNPDF3.1 set, $\alpha_{S}\left(m_{\mathrm{Z}}\right)=0.130$, whereas for the NLO and NNLO NNPDF3.1 sets, $\alpha_{S}\left(m_{\mathrm{Z}}\right)=0.118$. In general, the combination of contributions from multiple-parton interactions and parton-shower emissions is crucial to give a good description of variables measured in soft-collision events. The infrared threshold is relatively independent of center-of-mass energy when using NLO or NNLO PDF sets. Irrespective of the specific PDF used, predictions from the new tunes reproduce well the UE measurements at center-of-mass energies $\sqrt{s}=1.96$ and $7 \mathrm{TeV}$. A significant improvement in the description of UE measurements at $13 \mathrm{TeV}$ is observed with respect to predictions from old tunes that were extracted using data at lower collision energies. For the first time, predictions based on higher-order PDF sets are shown to give a reliable description of minimum-bias (MB) and UE mea- 

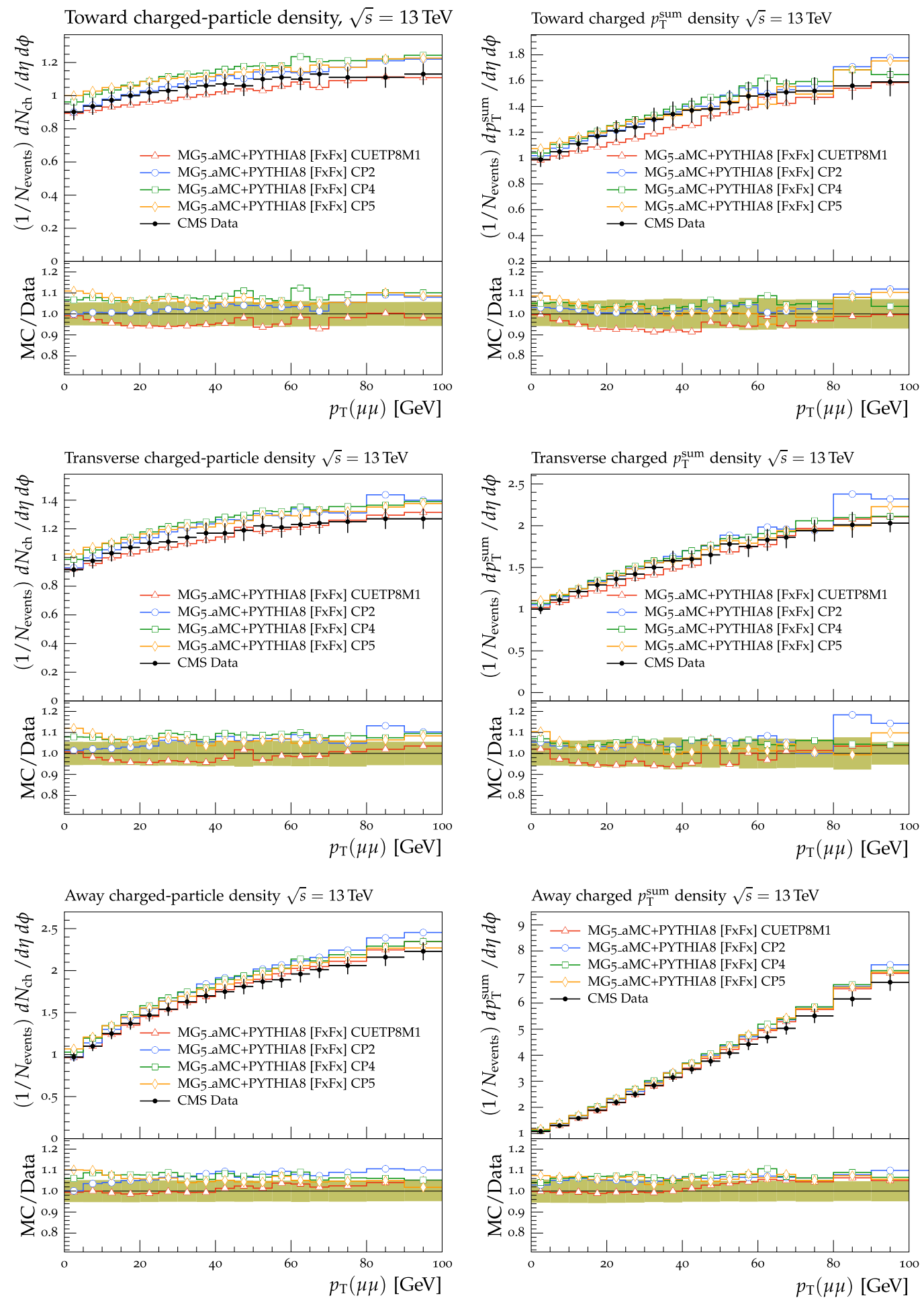

Fig. 21 The charged-particle multiplicity (left) and $p_{\mathrm{T}}^{\text {sum }}$ (right) in the toward (upper), transverse (middle), and away (lower) regions measured as a function of the $\mathrm{Z}$ boson $p_{\mathrm{T}}$ in Drell-Yan events at $\sqrt{s}=13 \mathrm{TeV}$ [21], and compared with the predictions obtained by an inclusive NLO ME calculated by MG5_aMC, interfaced to the UE simulation of PYTHIA8 with the CUETP8M1, CP2, CP4, and CP5 tunes. Tunes CP1 and CP3 are not shown in the plot but present a similar behaviour as, respectively,

tunes CP2 and CP4. The ratios of simulations to the data (MC/Data) are also shown, where the shaded band indicates the total experimental uncertainty of the data. Vertical lines drawn on the data points refer to the total uncertainty in the data. Vertical lines drawn on the MC points refer to the statistical uncertainty in the predictions. Horizontal bars indicate the associated bin width 
$\mathrm{Z}+$ jets, inclusive jet multiplicity, $\sqrt{s}=13 \mathrm{TeV}$
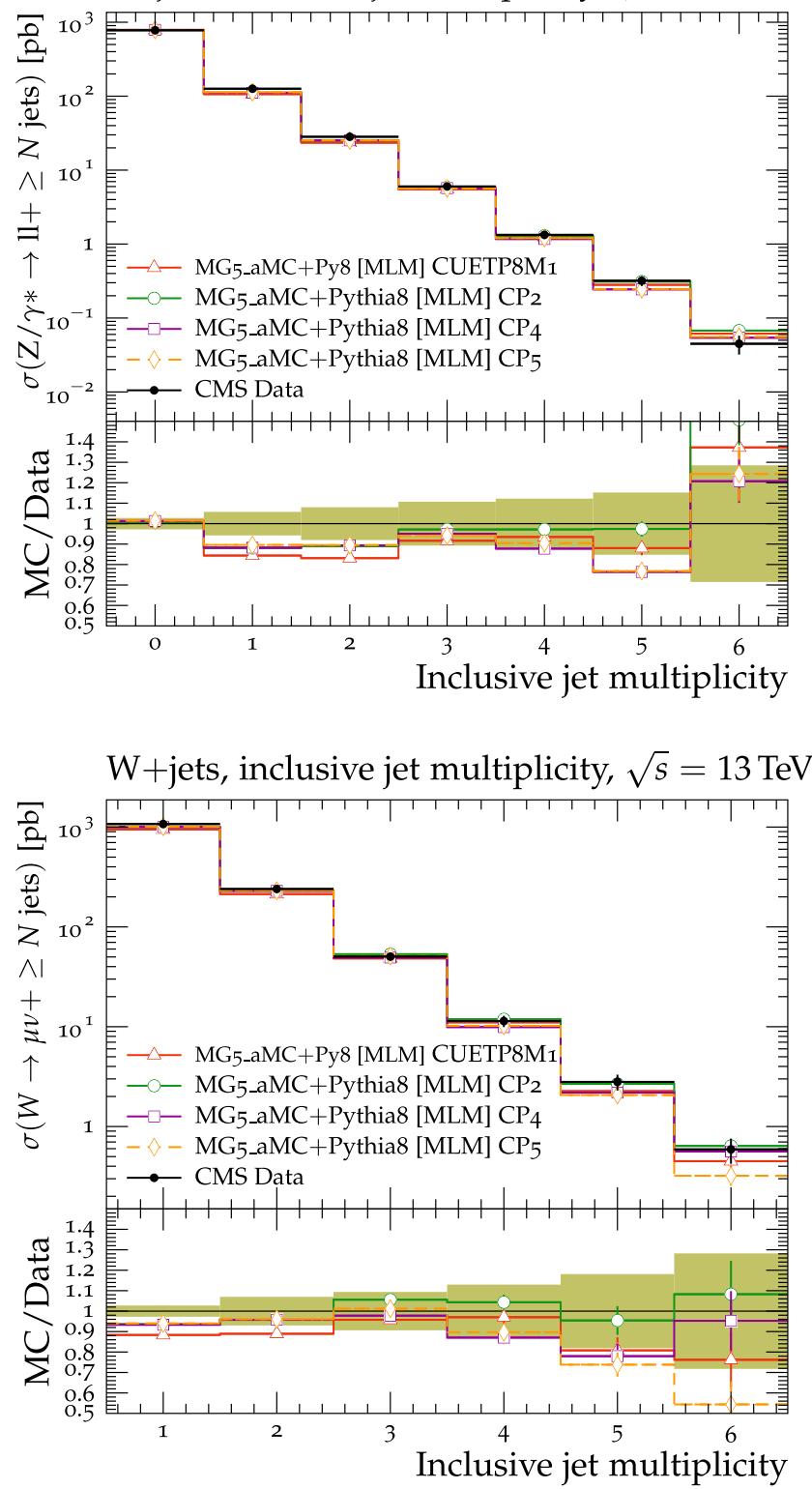

Fig. 22 Comparison with the measurement $[71,72]$ of the inclusive jet multiplicity in $\mathrm{Z}+$ jets (upper) and $\mathrm{W}+$ jets (lower) events predicted by MG5_aMC + PYTHIA8 with $k_{\mathrm{T}}-\mathrm{MLM}$ merging (left) and FxFx merging (right) for the different tunes. Tunes $\mathrm{CP} 1$ and $\mathrm{CP} 3$ are not shown in the plot but present a similar behaviour as, respectively, tunes CP2 and CP4. The ratios of simulations to the data (MC/Data) are also shown, where

surements, with a similar level of agreement as predictions from tunes using LO PDF sets.

Predictions of the new tunes agree well with the data for MB observables measured at pseudorapidities in the central $(|\eta|<2.4)$ and forward $(3.2<|\eta|<4.7)$ regions. The new CMS tunes simultaneously describe the number of charged particles produced in diffractive processes and $\mathrm{MB}$ collisions. Neither the new CMS tunes nor the CUETP8M1
$\mathrm{Z}+$ jets, inclusive jet multiplicity, $\sqrt{s}=13 \mathrm{TeV}$
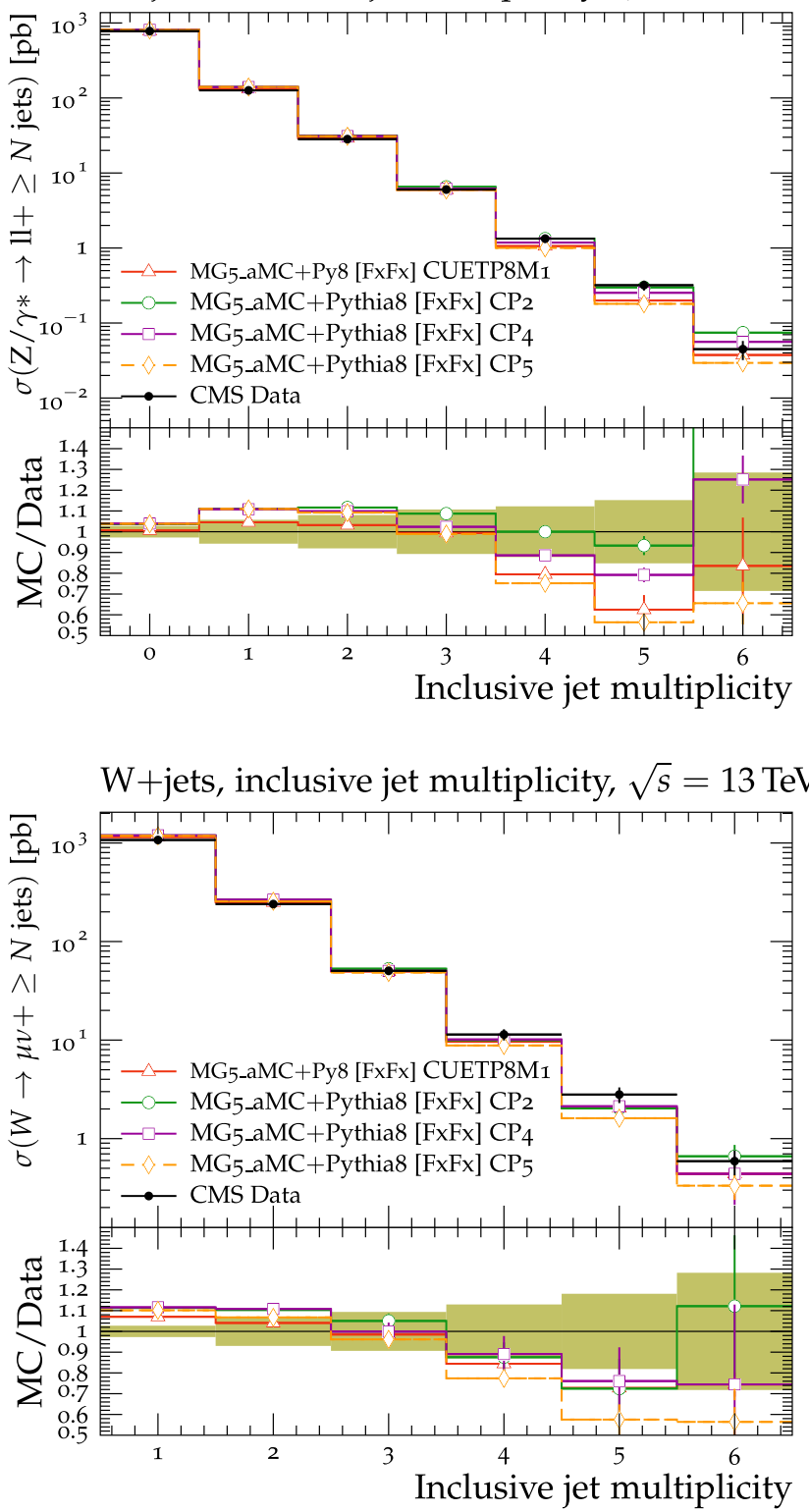

the shaded band indicates the total experimental uncertainty in the data. Vertical lines drawn on the data points refer to the total uncertainty in the data. Vertical lines drawn on the MC points refer to the statistical uncertainty in the predictions. Horizontal bars indicate the associated bin width

tune describe the very forward region $(-6.6<\eta<-5.2)$ well.

Measurements sensitive to double-parton scattering contributions are reproduced better by predictions using the $\mathrm{LO}$ PDF set in the UE simulation, without rapidity ordering of the initial-state shower.

The UE simulation provided by the new tunes can be interfaced to higher-order and multileg matrix element genera- 


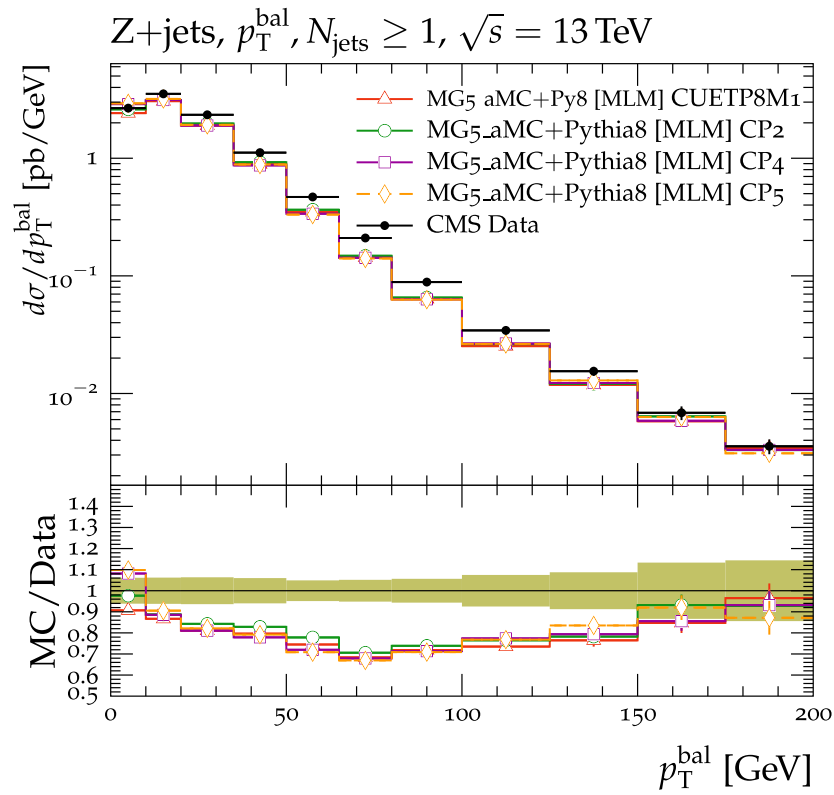

Fig. 23 Comparison with the measurement [71] of the $p_{\mathrm{T}}$ balance predicted by MG5_aMC + PYTHIA8 with $k_{\mathrm{T}}-$ MLM merging (left) and FxFx merging (right) for the different tunes for events with at least one jet. Tunes CP1 and CP3 are not shown in the plot but they present a similar behaviour as tunes $\mathrm{CP} 2$ and $\mathrm{CP} 4$, respectively. The ratios of simulations

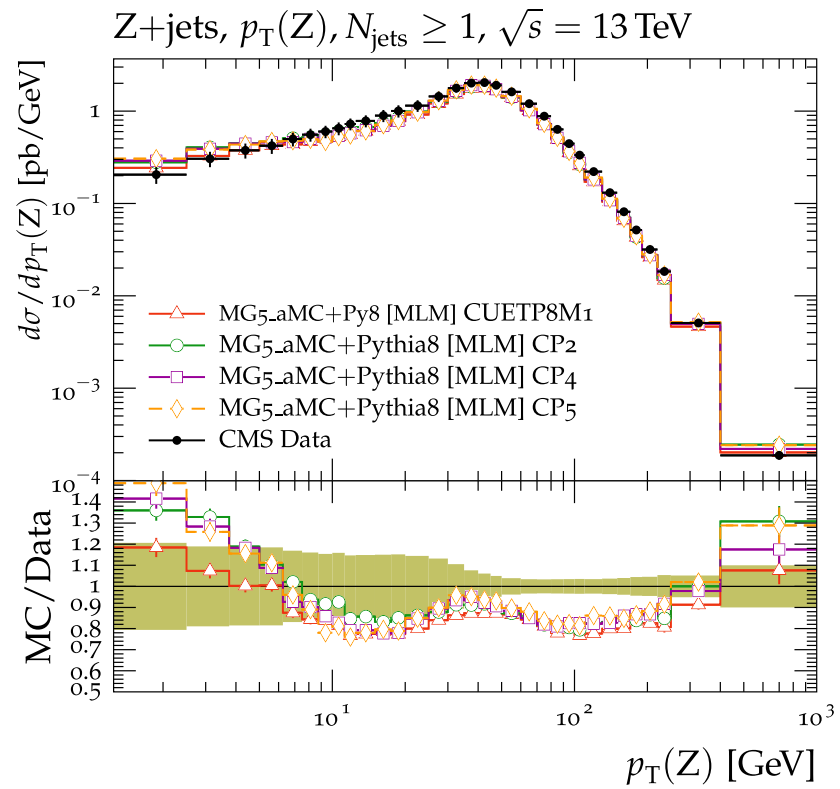

Fig. 24 Comparison with the measurement [71] of the $p_{\mathrm{T}}(\mathrm{Z})$ predicted by MG5_aMC + PYTHIA8 with $k_{\mathrm{T}}-\mathrm{MLM}$ merging (left) and FxFx merging (right) for the different tunes. Tunes CP1 and CP3 are not shown in the plot but they present a similar behaviour as tunes $\mathrm{CP} 2$ and $\mathrm{CP} 4$, respectively. The ratios of simulations to the data (MC/Data) are also

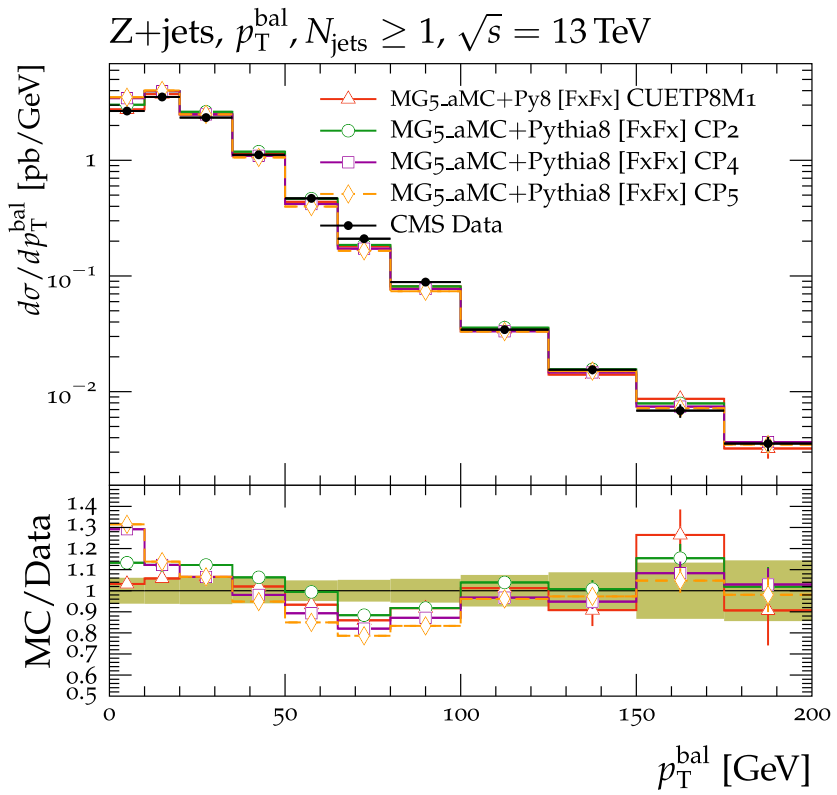

to the data (MC/Data) are also shown, where the shaded band indicates the total experimental uncertainty in the data. Vertical lines drawn on the data points refer to the total uncertainty in the data. Vertical lines drawn on the MC points refer to the statistical uncertainty in the predictions. Horizontal bars indicate the associated bin width

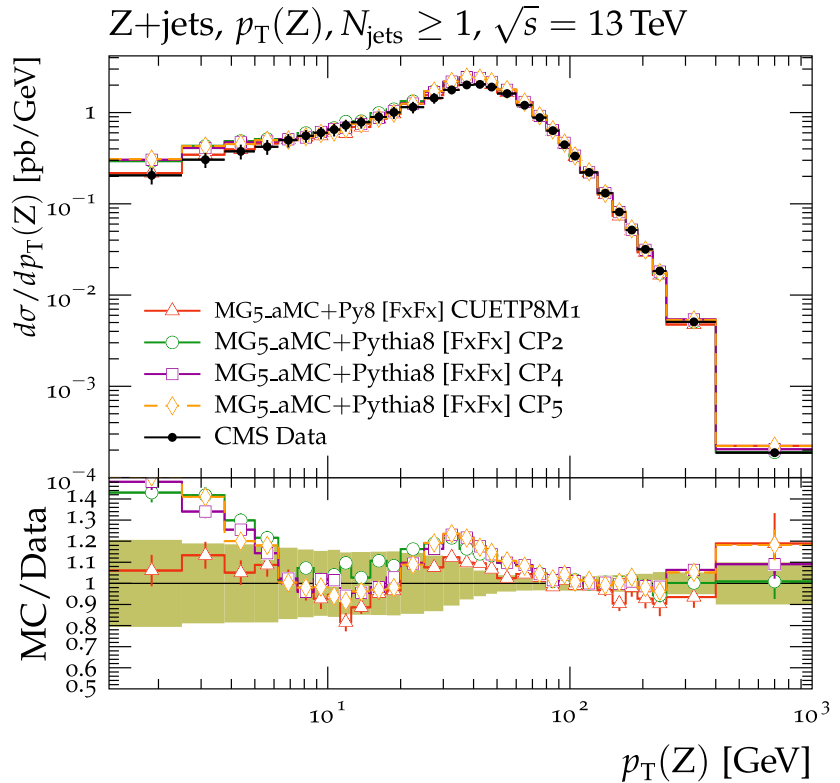

shown, where the shaded band indicates the total experimental uncertainty in data. Vertical lines drawn on the data points refer to the total uncertainty in the data. Vertical lines drawn on the MC points refer to the statistical uncertainty in the predictions. Horizontal bars indicate the associated bin width 
tors, such as POWHEG and MG5_aMC, without degrading the good description of UE observables. Such predictions also reproduce well observables measured in multijet final states, Drell-Yan, and top quark production processes. The central values of the normalized $t \bar{t}$ cross section in bins of the number of additional jets predicted by POWHEG + PYTHIA8 overestimate the data when a high value of $\alpha_{S}^{\mathrm{ISR}}\left(m_{\mathrm{Z}}\right) \gtrsim 0.130$ is used (CMS PYTHIA8 CP1 and CP2 tunes). Even when $\alpha_{S}^{\mathrm{ISR}}\left(m_{\mathrm{Z}}\right)=0.118$ is used, the CP4 tune overestimates the data at high jet multiplicities. This is cured by the rapidity ordering of the initial-state shower (CP5 tune). Measurements of azimuthal dijet correlations are also better described when a value of $\alpha_{S}^{\mathrm{ISR}}\left(m_{\mathrm{Z}}\right)=0.118$ is used in predictions obtained with POWHEG merged with PYTHIA8.

Comparisons with LEP event-shape observables and the distribution of the angle between two groomed subjets $\left(\Delta R_{g}\right)$ in $t \bar{t}$ events at the LHC show that in ME-PS merged configurations CMW rescaling is disfavored. It is also found that $\Delta R_{g}$ is better described by tunes with $\alpha_{S}^{\mathrm{FSR}}\left(m_{\mathrm{Z}}\right)$ higher than $\sim 0.120$ while LEP event-shape observables and UE event observables in $\mathrm{t}^{\bar{t}}$ events prefer a central value $\sim 0.120$ [22].

All of the new CMS tunes are supplied with their eigentunes, which can also be used to determine the uncertainties associated with the theoretical predictions. We show that predictions using the new tunes based on PDFs determined at LO, NLO, and NNLO agree reasonably well with the measurements, and that the new tunes can also be applied to LO and NLO calculations merged with parton showers, multipleparton interactions, and hadronization.

Acknowledgements Bundes-ministerium Forschungs-gemeinschaft Forschungs-zentren Rachada-pisek We congratulate our colleagues in the CERN accelerator departments for the excellent performance of the LHC and thank the technical and administrative staffs at CERN and at other CMS institutes for their contributions to the success of the CMS effort. In addition, we gratefully acknowledge the computing centres and personnel of the Worldwide LHC Computing Grid for delivering so effectively the computing infrastructure essential to our analyses. Finally, we acknowledge the enduring support for the construction and operation of the LHC and the CMS detector provided by the following funding agencies: the Austrian Federal Ministry of Education, Science and Research and the Austrian Science Fund; the Belgian Fonds de la Recherche Scientifique, and Fonds voor Wetenschappelijk Onderzoek; the Brazilian Funding Agencies (CNPq, CAPES, FAPERJ, FAPERGS, and FAPESP); the Bulgarian Ministry of Education and Science; CERN; the Chinese Academy of Sciences, Ministry of Science and Technology, and National Natural Science Foundation of China; the Colombian Funding Agency (COLCIENCIAS); the Croatian Ministry of Science, Education and Sport, and the Croatian Science Foundation; the Research Promotion Foundation, Cyprus; the Secretariat for Higher Education, Science, Technology and Innovation, Ecuador; the Ministry of Education and Research, Estonian Research Council via IUT23-4, IUT236 and PRG445 and European Regional Development Fund, Estonia; the Academy of Finland, Finnish Ministry of Education and Culture, and Helsinki Institute of Physics; the Institut National de Physique Nucléaire et de Physique des Particules / CNRS, and Commissariat à l'Énergie Atomique et aux Énergies Alternatives / CEA, France; the Bundesministerium für Bildung und Forschung, Deutsche Forschungs- gemeinschaft, and Helmholtz-Gemeinschaft Deutscher Forschungszentren, Germany; the General Secretariat for Research and Technology, Greece; the National Research, Development and Innovation Fund, Hungary; the Department of Atomic Energy and the Department of Science and Technology, India; the Institute for Studies in Theoretical Physics and Mathematics, Iran; the Science Foundation, Ireland; the Istituto Nazionale di Fisica Nucleare, Italy; the Ministry of Science, ICT and Future Planning, and National Research Foundation (NRF), Republic of Korea; the Ministry of Education and Science of the Republic of Latvia; the Lithuanian Academy of Sciences; the Ministry of Education, and University of Malaya (Malaysia); the Ministry of Science of Montenegro; the Mexican Funding Agencies (BUAP, CINVESTAV, CONACYT, LNS, SEP, and UASLP-FAI); the Ministry of Business, Innovation and Employment, New Zealand; the Pakistan Atomic Energy Commission; the Ministry of Science and Higher Education and the National Science Centre, Poland; the Fundação para a Ciência e a Tecnologia, Portugal; JINR, Dubna; the Ministry of Education and Science of the Russian Federation, the Federal Agency of Atomic Energy of the Russian Federation, Russian Academy of Sciences, the Russian Foundation for Basic Research, and the National Research Center "Kurchatov Institute"; the Ministry of Education, Science and Technological Development of Serbia; the Secretaría de Estado de Investigación, Desarrollo e Innovación, Programa Consolider-Ingenio 2010, Plan Estatal de Investigación Científica y Técnica y de Innovación 2013-2016, Plan de Ciencia, Tecnología e Innovación 2013-2017 del Principado de Asturias, and Fondo Europeo de Desarrollo Regional, Spain; the Ministry of Science, Technology and Research, Sri Lanka; the Swiss Funding Agencies (ETH Board, ETH Zurich, PSI, SNF, UniZH, Canton Zurich, and SER); the Ministry of Science and Technology, Taipei; the Thailand Center of Excellence in Physics, the Institute for the Promotion of Teaching Science and Technology of Thailand, Special Task Force for Activating Research and the National Science and Technology Development Agency of Thailand; the Scientific and Technical Research Council of Turkey, and Turkish Atomic Energy Authority; the National Academy of Sciences of Ukraine, and State Fund for Fundamental Researches, Ukraine; the Science and Technology Facilities Council, UK; the US Department of Energy, and the US National Science Foundation. Individuals have received support from the Marie-Curie programme and the European Research Council and Horizon 2020 Grant, contract Nos. 675440 and 765710 (European Union); the Leventis Foundation; the A.P. Sloan Foundation; the Alexander von Humboldt Foundation; the Belgian Federal Science Policy Office; the Fonds pour la Formation à la Recherche dans l'Industrie et dans l'Agriculture (FRIA-Belgium); the Agentschap voor Innovatie door Wetenschap en Technologie (IWTBelgium); the F.R.S.-FNRS and FWO (Belgium) under the "Excellence of Science - EOS" - be.h project n. 30820817; the Beijing Municipal Science \& Technology Commission, No. Z181100004218003; the Ministry of Education, Youth and Sports (MEYS) of the Czech Republic; the Lendület ("Momentum") Programme and the János Bolyai Research Scholarship of the Hungarian Academy of Sciences, the New National Excellence Program ÚNKP, the NKFIA research Grants 123842, 123959, 124845, 124850, 125105, 128713, 128786, and 129058 (Hungary); the Council of Scientific and Industrial Research, India; the HOMING PLUS programme of the Foundation for Polish Science, cofinanced from European Union, Regional Development Fund, the Mobility Plus programme of the Ministry of Science and Higher Education, the National Science Center (Poland), contracts Harmonia 2014/14/M/ST2/00428, Opus 2014/13/B/ST2/02543, 2014/15/B/ST2/03998, and 2015/19/B/ST2/02861, Sonata-bis 2012/07/ E/ST2/01406; the National Priorities Research Program by Qatar National Research Fund; the Programa de Excelencia María de Maeztu, and the Programa Severo Ochoa del Principado de Asturias; the Thalis and Aristeia programmes cofinanced by EU-ESF, and the Greek NSRF; the Rachadapisek Sompot Fund for Postdoctoral Fellowship, Chulalongkorn University, and the Chulalongkorn Academic into Its 2nd Cen- 
tury Project Advancement Project (Thailand); the Welch Foundation, contract C-1845; and the Weston Havens Foundation (USA).

Data Availability Statement This manuscript has no associated data or the data will not be deposited. [Author's comment: Release and preservation of data used by the CMS Collaboration as the basis for publications is guided by the CMS policy as written in its document "CMS data preservation, re-use and open access policy" (https://cmsdocdb.cern.ch/cgi-bin/PublicDocDB/RetrieveFile?docid= 6032\&filename $=$ CMSDataPolicyV1.2.pdf\&version=2).]

Open Access This article is licensed under a Creative Commons Attribution 4.0 International License, which permits use, sharing, adaptation, distribution and reproduction in any medium or format, as long as you give appropriate credit to the original author(s) and the source, provide a link to the Creative Commons licence, and indicate if changes were made. The images or other third party material in this article are included in the article's Creative Commons licence, unless indicated otherwise in a credit line to the material. If material is not included in the article's Creative Commons licence and your intended use is not permitted by statutory regulation or exceeds the permitted use, you will need to obtain permission directly from the copyright holder. To view a copy of this licence, visit http://creativecomm ons.org/licenses/by/4.0/.

Funded by SCOAP ${ }^{3}$.

\section{A Tables of tune uncertainties}

This section provides the values of the parameters corresponding to the uncertainties when the new CMS PYTHIA8 tunes are used. The tune uncertainty is obtained by extracting the eigentunes, which are defined by a change in the $\chi^{2}$ of the fit that equals the absolute $\chi^{2}$ value obtained in the tune. The eigentunes refer to the variations of the tunes along each of the maximally independent directions in the parameter space, obtained by using the covariance matrix in the region of the best tune. The number of directions defined in the parameter space equals the number of free parameters $n$ used in the fit and results into $2 n$ parameter variations, i.e., eigentunes. These variations represent a good set of systematic uncertainties in the given tune.

The estimations of the tune uncertainties, which have $2 n$ parameter variations, i.e., 10 for the new CMS PYTHIA 8 tunes, are very time consuming in analyses, since for each variation separate samples must be produced. Therefore, a lower number of variations is preferred. Hence, two variations, one "up" and one "down", are defined. For the definition of the two variations, predictions using the parameters of the eigentunes are implemented for the UE observables at $\sqrt{s}=13 \mathrm{TeV}$ and their differences with respect to the central predictions are added in quadrature. This procedure is applied in each bin and tune uncertainties are estimated without any correlation across the different bins. Positive differences between central predictions and tune variations define the upper edge of the bin-by-bin uncertainty, while negative differences define the lower edge of the bin-by-bin uncertainty. By following the same approach used for the extraction of the central values of the new CMS PYTHIA8 tunes, the upper edge is fitted to obtain the "up variation", while the "down variation" is obtained by fitting the lower edge. The parameters of the upand down-variations are listed in Table 6 for the tunes using LO PDF sets, and in Table 7 for the tunes using (N)NLO PDF sets. We checked that for a wide range of MB and UE observables at $\sqrt{s}=13 \mathrm{TeV}$ predictions from up- and downvariations, obtained by including the full set of eigenvalues, reproduce well the upper and the lower edge of the predic-

Table 6 Parameters of the "up-" and "down-" variation eigentunes for the PYTHIA8 CP1, and CP2 tunes

\begin{tabular}{|c|c|c|c|c|}
\hline PYTHIA8 parameter & $\begin{array}{l}\text { CP1 } \\
\text { Up }\end{array}$ & $\begin{array}{l}\text { CP1 } \\
\text { Down }\end{array}$ & $\begin{array}{l}\mathrm{CP} 2 \\
\mathrm{Up}\end{array}$ & $\begin{array}{l}\text { CP2 } \\
\text { Down }\end{array}$ \\
\hline MultipartonInteractions:pT0Ref (GeV) & 2.30 & 2.40 & 2.34 & 2.33 \\
\hline MultipartonInteractions: ecmPow & 0.15 & 0.15 & 0.14 & 0.14 \\
\hline MultipartonInteractions: coreFraction & 0.51 & 0.39 & 0.51 & 0.23 \\
\hline MultipartonInteractions: coreRadius & 0.58 & 0.60 & 0.41 & 0.34 \\
\hline ColourReconnection:range & 8.31 & 8.50 & 1.46 & 2.56 \\
\hline
\end{tabular}

Table 7 Parameters of the "up-" and "down-" variation eigentunes for the PYTHIA8 CP3, CP4, and CP5 tunes

\begin{tabular}{|c|c|c|c|c|c|c|}
\hline PYTHIA8 parameter & $\begin{array}{l}\text { CP3 } \\
\text { Up }\end{array}$ & $\begin{array}{l}\text { CP3 } \\
\text { Down }\end{array}$ & $\begin{array}{l}\text { CP4 } \\
\text { Up }\end{array}$ & $\begin{array}{l}\text { CP4 } \\
\text { Down }\end{array}$ & $\begin{array}{l}\text { CP5 } \\
\text { Up }\end{array}$ & $\begin{array}{l}\text { CP5 } \\
\text { Down }\end{array}$ \\
\hline MultipartonInteractions:pT0Ref $(\mathrm{GeV})$ & 1.48 & 1.54 & 1.48 & 1.54 & 1.41 & 1.46 \\
\hline MultipartonInteractions: ecmPow & 0.02 & 0.02 & 0.02 & 0.02 & 0.03 & 0.03 \\
\hline MultipartonInteractions: coreFraction & 0.35 & 0.25 & 0.36 & 0.33 & 0.43 & 0.73 \\
\hline MultipartonInteractions: coreRadius & 0.49 & 0.35 & 0.58 & 0.57 & 0.67 & 0.69 \\
\hline ColourReconnection:range & 8.15 & 3.96 & 7.93 & 6.88 & 4.88 & 4.69 \\
\hline
\end{tabular}


Table 8 The correlation matrix, retrieved when extracting the CP5 tune. This is obtained by evaluating the correlation values of the parameter variations obtained in the eigentunes

\begin{tabular}{lcccrr}
\hline & pToRef & ecmPow & coreFraction & coreRadius & range \\
\hline pT0Ref & 1.00 & -0.21 & -0.19 & -0.19 & 0.15 \\
ecmPow & -0.21 & 1.00 & 0.30 & 0.69 & -0.21 \\
coreFraction & -0.19 & 0.30 & 1.00 & 0.32 & -0.64 \\
coreRadius & -0.19 & 0.69 & 0.32 & -0.52 & -0.52 \\
range & 0.15 & -0.21 & -0.64 & -0.00 \\
\hline
\end{tabular}

Table 9 "Up" and "Down" ISR and FSR variations for CP5 when $\alpha_{S}^{\mathrm{ISR}}\left(m_{\mathrm{Z}}\right)$ or $\alpha_{S}^{\mathrm{FSR}}\left(m_{\mathrm{Z}}\right)$ is treated as a free parameter

\begin{tabular}{lllll}
\hline PYTHIA8 parameter & Central & Up & Down & $\chi^{2} /$ dof \\
\hline$\alpha_{S}^{\text {ISR }}\left(m_{Z}\right)$ value & 0.121 & 0.128 & 0.114 & 0.75 \\
$\alpha_{S}^{\text {FSR }}\left(m_{Z}\right)$ value & 0.119 & 0.122 & 0.115 & 0.78 \\
\hline
\end{tabular}

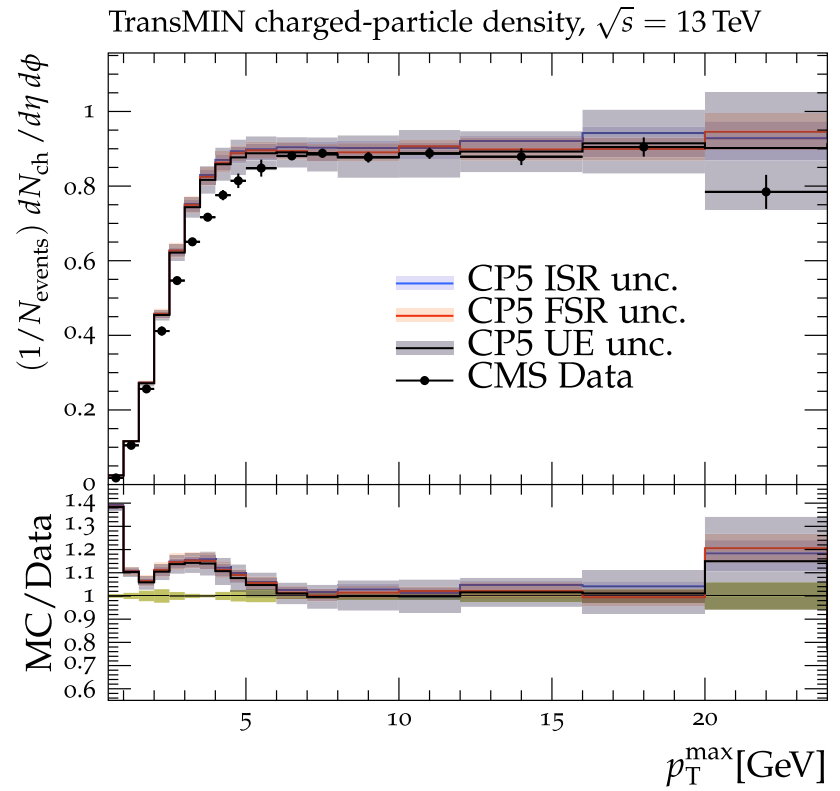

Fig. 25 The variations allowed by the CP5 tune when $\alpha_{S}^{\mathrm{ISR}}\left(m_{\mathrm{Z}}\right)$ (blue band) and $\alpha_{S}^{\mathrm{FSR}}\left(m_{\mathrm{Z}}\right)$ (red band) are left free in the fit for chargedparticle (left) and charged $p_{\mathrm{T}}^{\text {sum }}$ (right) density in the transMIN region at $\sqrt{s}=13 \mathrm{TeV}$. Vertical lines drawn on the data points refer to the

tions. Hence, tune uncertainties estimated by evaluating predictions of up- and down-variations represent a reliable way of estimating the systematic uncertainties in the tunes. The correlation matrix for the fit of the CP5 tune is displayed in Table 8 . It is retrieved by evaluating the correlation of the parameter variations obtained in the eigentunes.

Variations of the values of the ISR and FSR are also studied, in order to check the consistency of the selected $\alpha_{S}^{\mathrm{ISR}}\left(m_{\mathrm{Z}}\right)$ and $\alpha_{S}^{\mathrm{FSR}}\left(m_{\mathrm{Z}}\right)$ values selected for the tunes and to estimate the allowed range of $\alpha_{S}^{\mathrm{ISR}}\left(m_{\mathrm{Z}}\right)$ and $\alpha_{S}^{\mathrm{FSR}}\left(m_{\mathrm{Z}}\right)$ values in the PS using the CP5 tune. Starting from tune CP5, the

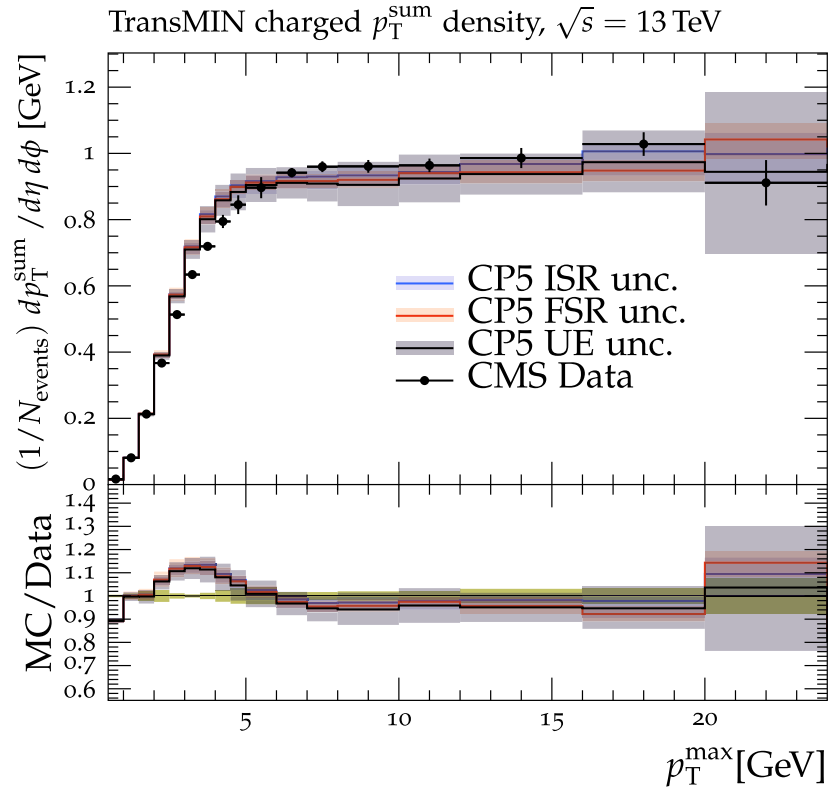

total uncertainty in the data. The grey band represents the total UE uncertainty for the tune CP5. Horizontal bars indicate the associated bin width

value of $\alpha_{S}^{\text {ISR }}\left(m_{\mathrm{Z}}\right)$ is fitted to UE observables measured by $\mathrm{CMS}$ at $\sqrt{s}=13 \mathrm{TeV}$. The same procedure is repeated when $\alpha_{S}^{\mathrm{FSR}}\left(m_{\mathrm{Z}}\right)$ is fitted. The parameters obtained from the fits are shown in Table 9, along with the up and down variation.

Figure 25 shows the predictions of the CP5 tune, with the corresponding variation bands relative to the UE parameters, and the $\alpha_{S}^{\mathrm{ISR}}\left(m_{\mathrm{Z}}\right)$ and $\alpha_{S}^{\mathrm{FSR}}\left(m_{\mathrm{Z}}\right)$ values for the chargedparticle and $p_{\mathrm{T}}^{\text {sum }}$ densities at $\sqrt{s}=13 \mathrm{TeV}$ in the transMIN region. 


\section{References}

1. T. Sjöstrand et al., An introduction to Pythia 8.2. Comput. Phys. Commun. 191, 159 (2015). https://doi.org/10.1016/j.cpc.2015.01. 024. arXiv: 1410.3012

2. M. Bahr et al., Herwig++ physics and manual. Eur. Phys. J. C 58, 639 (2008). https://doi.org/10.1140/epjc/s10052-008-0798-9. arXiv:0803.0883

3. J. Bellm et al., Herwig 7.0/Herwig++ 3.0 release note. Eur. Phys. J. C 76, 196 (2016). https://doi.org/10.1140/epjc/ s10052-016-4018-8. arXiv:1512.01178

4. T. Gleisberg et al., Event generation with SHERPA 1.1. JHEP 02, 7 (2009). https://doi.org/10.1088/1126-6708/2009/02/007. arXiv:0811.4622

5. CMS Collaboration, Event generator tunes obtained from underlying event and multiparton scattering measurements. Eur. Phys. J. C 76, 155 (2016). https://doi.org/10.1140/epjc/s10052-016-3988-x. arXiv: 1512.00815

6. P. Skands, S. Carrazza, J. Rojo, Tuning PYTHIA 8.1: the Monash 2013 tune. Eur. Phys. J. C 74, 3024 (2014). https://doi.org/10.1140/ epjc/s10052-014-3024-y. arXiv:1404.5630

7. S. Carrazza, S. Forte, J. Rojo, Parton distributions and event generators, In Proceedings of the 43rd International Symposium on Multiparticle Dynamics (ISMD 13). 2013. arXiv:1311.5887

8. R. Corke, T. Sjostrand, Interleaved parton showers and tuning prospects. JHEP 03, 032 (2011). https://doi.org/10.1007/ JHEP03(2011)032. arXiv:1011.1759

9. CDF Collaboration, Study of the energy dependence of the underlying event in proton-antiproton collisions. Phys. Rev. D 92, 092009 (2015). https://doi.org/10.1103/PhysRevD.92.092009. arXiv: 1508.05340

10. CMS Collaboration, Measurement of the underlying event activity in pp collisions at the LHC at $7 \mathrm{TeV}$ and comparison with $0.9 \mathrm{TeV}$. CMS Physics Analysis Summary CMS-PAS-FSQ-12-020 (2012)

11. J. Pumplin et al., New generation of parton distributions with uncertainties from global QCD analysis. JHEP 07, 12 (2002). https://doi. org/10.1088/1126-6708/2002/07/012. arXiv:hep-ph/0201195

12. ATLAS Collaboration, ATLAS PYTHIA 8 tunes to $7 \mathrm{TeV}$ data. Technical Report ATL-PHYS-PUB-2014-021 (2015)

13. ATLAS Collaboration, Summary of ATLAS PYTHIA 8 tunes (2012)

14. ATLAS Collaboration, Measurement of the $Z / \gamma^{*}$ boson transverse momentum distribution in $p p$ collisions at $\sqrt{s}=7 \mathrm{TeV}$ with the ATLAS detector. JHEP 09, 145 (2014). https://doi.org/10.1007/ JHEP09(2014)145. arXiv:1406.3660

15. N. Fischer, T. Sjöstrand, Thermodynamical string fragmentation. JHEP 01, 140 (2017). https://doi.org/10.1007/JHEP01(2017)140. arXiv: 1610.09818

16. CMS Collaboration, Underlying event measurements with leading particles and jets in pp collisions at $\sqrt{s}=13 \mathrm{TeV}$. CMS Physics Analysis Summary CMS-PAS-FSQ-15-007 (2015)

17. NNPDF Collaboration, Parton distributions from high-precision collider data. Eur. Phys. J. C 77, 663 (2017). https://doi.org/10. 1140/epjc/s10052-017-5199-5. arXiv:1706.00428

18. CMS Collaboration, Pseudorapidity distribution of charged hadrons in proton-proton collisions at $\sqrt{s}=13 \mathrm{TeV}$. Phys. Lett. B 751, 143 (2015). https://doi.org/10.1016/j.physletb.2015.10.004. arXiv: 1507.05915

19. P.D.B. Collins, An introduction to Regge theory and high-energy physics. Cambridge Monographs on Mathematical Physics. Cambridge University Press, Cambridge (2009). https://doi.org/10. 1017/CBO9780511897603 (ISBN 9780521110358 )

20. CMS Collaboration, Measurement of the underlying event in the Drell-Yan process in proton-proton collisions at $\sqrt{s}=7 \mathrm{TeV}$.
Eur. Phys. J. C 72, 2080 (2012). https://doi.org/10.1140/epjc/ s10052-012-2080-4. arXiv:1204.1411

21. CMS Collaboration, Measurement of the underlying event activity in inclusive $\mathrm{Z}$ boson production in proton-proton collisions at $\sqrt{s}=13 \mathrm{TeV}$. JHEP 07, 32 (2018). https://doi.org/10.1007/ JHEP07(2018)032. arXiv:1711.04299

22. CMS Collaboration, Study of the underlying event in top quark pair production in pp collisions at $13 \mathrm{TeV}$. Eur. Phys. J. C 79, 123 (2019). https://doi.org/10.1140/epjc/s10052-019-6620-z. arXiv: 1807.02810

23. S. Navin, Diffraction in PYTHIA (2010). arXiv:1005.3894

24. CMS Collaboration, Measurement of charged particle spectra in minimum-bias events from proton-proton collisions at $\sqrt{s}=$ 13 TeV. Eur. Phys. J. C 78, 697 (2018). https://doi.org/10.1140/ epjc/s10052-018-6144-y. arXiv:1806.11245

25. CMS Collaboration, Measurement of normalized differential $t \bar{t}$ cross sections in the dilepton channel from pp collisions at $\sqrt{s}=13$ TeV. JHEP 04, 60 (2018). https://doi.org/10.1007/ JHEP04(2018)060. arXiv: 1708.07638

26. CMS Collaboration, Investigations of the impact of the parton shower tuning in PYTHIA 8 in the modelling of $\mathrm{tt}_{\mathrm{t}}$ at $\sqrt{s}=8$ and 13 TeV. CMS Physics Analysis Summary CMS-PAS-TOP-16-021 (2016)

27. ATLAS Collaboration, Measurement of charged-particle distributions sensitive to the underlying event in $\sqrt{s}=13 \mathrm{TeV}$ proton-proton collisions with the ATLAS detector at the LHC. JHEP 03, 157 (2017). https://doi.org/10.1007/JHEP03(2017)157. arXiv: 1701.05390

28. P. Gunnellini, H. Jung, R. Maharucksit, Investigation of the energy dependence of the $p_{\text {T0 }}$ parameter in the PYTHIA 8 Monte Carlo event generator. Eur. Phys. J. C 78, 521 (2018). https://doi.org/10. 1140/epjc/s10052-018-6004-9. arXiv:1801.02536

29. L.A. Harland-Lang, A.D. Martin, P. Motylinski, R.S. Thorne, Parton distributions in the LHC era: MMHT 2014 PDFs. Eur. Phys. J. C 75, 204 (2015). https://doi.org/10.1140/epjc/s10052-015-3397-6. arXiv: 1412.3989

30. S. Gieseke, F. Loshaj, P. Kirchgaeber, Soft and diffractive scattering with the cluster model in Herwig. Eur. Phys. J. C 77, 156 (2017). https://doi.org/10.1140/epjc/s10052-017-4727-7. arXiv:1612.04701

31. J. Alwall et al., Comparative study of various algorithms for the merging of parton showers and matrix elements in hadronic collisions. Eur. Phys. J. C 53, 473 (2008). https://doi.org/10.1140/epjc/ s10052-007-0490-5. arXiv:0706.2569

32. S. Catani, F. Krauss, R. Kuhn, B.R. Webber, QCD matrix elements + parton showers. JHEP 11, 063 (2001). https://doi.org/10.1088/ 1126-6708/2001/11/063. arXiv:hep-ph/0109231

33. F. Krauss, Matrix elements and parton showers in hadronic interactions. JHEP 08, 015 (2002). https://doi.org/10.1088/1126-6708/ 2002/08/015. arXiv:hep-ph/0205283

34. B. Cooper et al., Importance of a consistent choice of $\alpha_{S}$ in the matching of AlpGen and PYTHIA. Eur. Phys. J. C 72, 2078 (2012). https://doi.org/10.1140/epjc/s10052-012-2078-y. arXiv: 1109.5295

35. Particle Data Group Collaboration, Review of particle physics. Phys. Rev. D 98, 30001 (2018). https://doi.org/10.1103/PhysRevD. 98.030001

36. S. Alioli, P. Nason, C. Oleari, E. Re, A general framework for implementing NLO calculations in shower Monte Carlo programs: the POWHEG BOX. JHEP 06, 43 (2010). https://doi.org/10.1007/ JHEP06(2010)043. arXiv:1002.2581

37. J. Alwall et al., The automated computation of tree-level and nextto-leading order differential cross sections, and their matching to parton shower simulations. JHEP 07, 79 (2014). https://doi.org/10. 1007/JHEP07(2014)079. arXiv:1405.0301 
38. R. Frederix, S. Frixione, Merging meets matching in MC@NLO. JHEP 12, 61 (2012). https://doi.org/10.1007/JHEP12(2012)061. arXiv: 1209.6215

39. S. Frixione, P. Nason, C. Oleari, Matching NLO QCD computations with parton shower simulations: the POWHEG method. JHEP 11, 70 (2007). https://doi.org/10.1088/1126-6708/2007/11/ 070. arXiv:0709.2092

40. T. Sjöstrand, M. van Zijl, A multiple interaction model for the event structure in hadron collisions. Phys. Rev. D 36, 2019 (1987). https://doi.org/10.1103/PhysRevD.36.2019

41. T. Sjöstrand, P.Z. Skands, Multiple interactions and the structure of beam remnants. JHEP 03, 53 (2004). https://doi.org/10.1088/ 1126-6708/2004/03/053. arXiv:hep-ph/0402078

42. A. Buckley, J. Butterworth, D. Grellscheid, H. Hoeth, L. Lönnblad, J. Monk, H. Schulze, F. Siegert, Rivet user manual. Comput. Phys. Commun. 184, 2803 (2013). https://doi.org/10.1016/j.cpc.2013. 05.021. arXiv: 1003.0694

43. A. Buckley et al., Systematic event generator tuning for the LHC. Eur. Phys. J. C 65, 331 (2010). https://doi.org/10.1140/epjc/ s10052-009-1196-7. arXiv:0907.2973

44. G.A. Schuler, T. Sjöstrand, Hadronic diffractive cross sections and the rise of the total cross section. Phys. Rev. D 49, 2257 (1994). https://doi.org/10.1103/PhysRevD.49.2257

45. ALEPH Collaboration, Studies of QCD at e+e- entre-of-mass energies between $91 \mathrm{GeV}$ and $209 \mathrm{GeV}$. Eur. Phys. J. C 35, 457 (2004). https://doi.org/10.1140/epjc/s2004-01891-4

46. S. Catani, B.R. Webber, G. Marchesini, QCD coherent branching and semiinclusive processes at large x. Nucl. Phys. B 349, 635 (1991). https://doi.org/10.1016/0550-3213(91)90390-J

47. C.M.S. Collaboration, Measurement of the energy density as a function of pseudorapidity in proton-proton collisions at $\sqrt{s}=$ 13 TeV. Eur. Phys. J. C 79, 391 (2019). https://doi.org/10.1140/ epjc/s10052-019-6861-x. arXiv:1812.04095

48. CMS Collaboration, The CMS Experiment at the CERN LHC. JINST 3, S08004 (2008). https://doi.org/10.1088/1748-0221/3/08/ S08004

49. CMS Collaboration, Measurement of the inclusive energy spectrum in the very forward direction in proton-proton collisions at $\sqrt{s}=13 \mathrm{TeV}$. JHEP 08, 46 (2017). https://doi.org/10.1007/ JHEP08(2017)046. arXiv:1701.08695

50. CMS Collaboration, Measurement of the inelastic proton-proton cross section at $\sqrt{s}=13 \mathrm{TeV}$. JHEP 07, 161 (2018). https://doi. org/10.1007/JHEP07(2018)161. arXiv:1802.02613

51. A. Donnachie, P.V. Landshoff, Elastic scattering and diffraction dissociation. Nucl. Phys. B 244, 322 (1984). https://doi.org/10. 1016/0550-3213(84)90315-8

52. R. Ciesielski, K. Goulianos, MBR Monte Carlo simulation in PYTHIA 8, In Proceedings of the 36th International Conference on High Energy Physics (ICHEP2012), p. 301. Melbourne. 2013. arXiv:1205.1446. (PoS(DIS 2013)091). https://doi.org/10.22323/ 1.191 .0091

53. S. Alioli et al., Jet pair production in POWHEG. JHEP 04, 81 (2011). https://doi.org/10.1007/JHEP04(2011)081. arXiv: 1012.3380

54. CMS Collaboration, Determination of jet energy calibration and transverse momentum resolution in CMS. JINST 6, 11002 (2011). https://doi.org/10.1088/1748-0221/6/11/P11002. arXiv: 1107.4277

55. CMS Collaboration, Jet energy scale and resolution in the CMS experiment in pp collisions at $8 \mathrm{TeV}$. JINST 12, P02014 (2017). https://doi.org/10.1088/1748-0221/12/02/ P02014. arXiv:1607.03663

56. CMS Collaboration, Measurement of the double-differential inclusive jet cross section in proton-proton collisions at $\sqrt{s}=13 \mathrm{TeV}$. Eur. Phys. J. C 76, 451 (2016). https://doi.org/10.1140/epjc/ s10052-016-4286-3. arXiv:1605.04436
57. CMS Collaboration, Azimuthal correlations for inclusive 2-jet, 3jet, and 4-jet events in pp collisions at $\sqrt{s}=13$ TeV. Eur. Phys. J. C 78, 566 (2018). https://doi.org/10.1140/epjc/s10052-018-6033-4. arXiv: 1712.05471

58. M. Cacciari, G.P. Salam, G. Soyez, The anti- $k_{\mathrm{T}}$ jet clustering algorithm. JHEP 04, 63 (2008). https://doi.org/10.1088/1126-6708/ 2008/04/063. arXiv:0802.1189

59. M. Cacciari, G.P. Salam, G. Soyez, FastJet user manual. Eur. Phys. J. C 72, 1896 (2012). https://doi.org/10.1140/epjc/ s10052-012-1896-2. arXiv:1111.6097

60. D0 Collaboration, Measurement of dijet azimuthal decorrelations at central rapidities in $p \bar{p}$ collisions at $\sqrt{s}=1.96 \mathrm{TeV}$. Phys. Rev. Lett. 94, 221801 (2005). https://doi.org/10.1103/PhysRevLett.94. 221801

61. CMS Collaboration, Measurement of four-jet production in proton-proton collisions at $\sqrt{s}=7$ TeV. Phys. Rev. D 89, 092010 (2014). https://doi.org/10.1103/PhysRevD.89.092010. arXiv: 1312.6440

62. ATLAS Collaboration, Measurement of the inelastic proton-proton cross section at $\sqrt{s}=13 \mathrm{TeV}$ with the ATLAS detector at the LHC. Phys. Rev. Lett. 117, 182002 (2016). https://doi.org/10. 1103/PhysRevLett.117.182002. arXiv:1606.02625

63. CMS Collaboration, Studies of inclusive four-jet production with two b-tagged jets in proton-proton collisions at $7 \mathrm{TeV}$. Phys. Rev. D 94, 112005 (2016). https://doi.org/10.1103/PhysRevD.94.112005. arXiv: 1609.03489

64. B. Humpert, R. Odorico, Multi-parton scattering and QCD radiation as sources of four-jet events. Phys. Lett. B 154, 211 (1985). https://doi.org/10.1016/0370-2693(85)90587-8

65. M. Mangano, Four-jet production at the tevatron collider. Z. Phys. C 42, 331 (1989). https://doi.org/10.1007/BF01555875

66. CMS Collaboration, Probing color coherence effects in pp collisions at $\sqrt{s}=7 \mathrm{TeV}$. Eur. Phys. J. C 74 (2014). https://doi.org/10. 1140/epjc/s10052-014-2901-8. arXiv:1311.5815

67. P. Gunnellini, Study of double parton scattering using four-jet scenarios in proton-proton collisions at $\sqrt{s}=7 \mathrm{TeV}$ with the CMS experiment at the Large Hadron Collider. PhD thesis, U. Hamburg, Dept. Phys. (2014). https://doi.org/10.1007/978-3-319-22213-4

68. P. Nason, A new method for combining NLO QCD with shower Monte Carlo algorithms. JHEP 11, 40 (2004). https://doi.org/10. 1088/1126-6708/2004/11/040. arXiv:hep-ph/0409146

69. CMS Collaboration, Measurement of differential cross sections for top quark pair production using the lepton+jets final state in protonproton collisions at 13 TeV. Phys. Rev. D 95, 092001 (2017). https:// doi.org/10.1103/PhysRevD.95.092001. arXiv:1610.04191

70. CMS Collaboration, Measurement of jet substructure observables in $\mathrm{t}_{\mathrm{t}}$ events from proton-proton collisions at $\sqrt{s}=13 \mathrm{TeV}$. Phys. Rev. D 98, 092014 (2018). https://doi.org/10.1103/PhysRevD.98. 092014. arXiv: 1808.07340

71. CMS Collaboration, Measurement of differential cross sections for $\mathrm{Z}$ boson production in association with jets in proton-proton collisions at $\sqrt{s}=13$ TeV. Eur. Phys. J. C 78, 965 (2018). https:// doi.org/10.1140/epjc/s10052-018-6373-0. arXiv:1804.05252

72. CMS Collaboration, Measurement of the differential cross sections for the associated production of a $W$ boson and jets in protonproton collisions at $\sqrt{s}=13 \mathrm{TeV}$. Phys. Rev. D 96, 72005 (2017). https://doi.org/10.1103/PhysRevD.96.072005. arXiv:1707.05979 


\section{CMS Collaboration}

Yerevan Physics Institute, Yerevan, Armenia

A. M. Sirunyan, A. Tumasyan

\section{Institut für Hochenergiephysik, Wien, Austria}

W. Adam, F. Ambrogi, E. Asilar, T. Bergauer, J. Brandstetter, M. Dragicevic, J. Erö, A. Escalante Del Valle, M. Flechl, R. Frühwirth ${ }^{1}$, V. M. Ghete, J. Hrubec, M. Jeitler ${ }^{1}$, N. Krammer, I. Krätschmer, D. Liko, T. Madlener, I. Mikulec, N. Rad, H. Rohringer, J. Schieck ${ }^{1}$, R. Schöfbeck, M. Spanring, D. Spitzbart, W. Waltenberger, J. Wittmann, C.-E. Wulz ${ }^{1}$,

M. Zarucki

Institute for Nuclear Problems, Minsk, Belarus

V. Chekhovsky, V. Mossolov, J. Suarez Gonzalez

Universiteit Antwerpen, Antwerpen, Belgium

E. A. De Wolf, D. Di Croce, X. Janssen, J. Lauwers, M. Pieters, H. Van Haevermaet, P. Van Mechelen, N. Van Remortel

Vrije Universiteit Brussel, Brussel, Belgium

S. Abu Zeid, F. Blekman, J. D’Hondt, J. De Clercq, K. Deroover, G. Flouris, D. Lontkovskyi, S. Lowette, I. Marchesini, S. Moortgat, L. Moreels, Q. Python, K. Skovpen, S. Tavernier, W. Van Doninck, P. Van Mulders, I. Van Parijs

Université Libre de Bruxelles, Bruxelles, Belgium

D. Beghin, B. Bilin, H. Brun, B. Clerbaux, G. De Lentdecker, H. Delannoy, B. Dorney, G. Fasanella, L. Favart, R. Goldouzian, A. Grebenyuk, A. K. Kalsi, T. Lenzi, J. Luetic, N. Postiau, E. Starling, L. Thomas, C. Vander Velde,

P. Vanlaer, D. Vannerom, Q. Wang

\section{Ghent University, Ghent, Belgium}

T. Cornelis, D. Dobur, A. Fagot, M. Gul, I. Khvastunov², D. Poyraz, C. Roskas, D. Trocino, M. Tytgat, W. Verbeke,

B. Vermassen, M. Vit, N. Zaganidis

\section{Université Catholique de Louvain, Louvain-la-Neuve, Belgium}

H. Bakhshiansohi, O. Bondu, S. Brochet, G. Bruno, C. Caputo, P. David, C. Delaere, M. Delcourt, A. Giammanco,

G. Krintiras, V. Lemaitre, A. Magitteri, K. Piotrzkowski, A. Saggio, M. Vidal Marono, P. Vischia, S. Wertz, J. Zobec

Centro Brasileiro de Pesquisas Fisicas, Rio de Janeiro, Brazil

F. L. Alves, G. A. Alves, M. Correa Martins Junior, G. Correia Silva, C. Hensel, A. Moraes, M. E. Pol, P. Rebello Teles

Universidade do Estado do Rio de Janeiro, Rio de Janeiro, Brazil

E. Belchior Batista Das Chagas, W. Carvalho, J. Chinellato ${ }^{3}$, E. Coelho, E. M. Da Costa, G. G. Da Silveira ${ }^{4}$,

D. De Jesus Damiao, C. De Oliveira Martins, S. Fonseca De Souza, H. Malbouisson, D. Matos Figueiredo,

M. Melo De Almeida, C. Mora Herrera, L. Mundim, H. Nogima, W. L. Prado Da Silva, L. J. Sanchez Rosas, A. Santoro, A. Sznajder, M. Thiel, E. J. Tonelli Manganote ${ }^{3}$, F. Torres Da Silva De Araujo, A. Vilela Pereira

Universidade Estadual Paulista ${ }^{a}$, Universidade Federal do $\mathrm{ABC}^{b}$, São Paulo, Brazil

S. Ahuja ${ }^{a}$, C. A. Bernardes ${ }^{a}$, L. Calligaris ${ }^{a}$, T. R. Fernandez Perez Tomei ${ }^{a}$, E. M. Gregores $^{b}$, P. G. Mercadante ${ }^{b}$, $^{2}$

S. F. Novaes ${ }^{a}$, SandraS. Padula ${ }^{a}$

Institute for Nuclear Research and Nuclear Energy, Bulgarian Academy of Sciences, Sofia, Bulgaria

A. Aleksandrov, R. Hadjiiska, P. Iaydjiev, A. Marinov, M. Misheva, M. Rodozov, M. Shopova, G. Sultanov

University of Sofia, Sofia, Bulgaria

A. Dimitrov, L. Litov, B. Pavlov, P. Petkov

Beihang University, Beijing, China

W. Fang ${ }^{5}$, X. Gao ${ }^{5}$, L. Yuan

Institute of High Energy Physics, Beijing, China

M. Ahmad, J. G. Bian, G. M. Chen, H. S. Chen, M. Chen, Y. Chen, C. H. Jiang, D. Leggat, H. Liao, Z. Liu,

S. M. Shaheen ${ }^{6}$, A. Spiezia, J. Tao, Z. Wang, E. Yazgan, H. Zhang, S. Zhang ${ }^{6}$, J. Zhao 
State Key Laboratory of Nuclear Physics and Technology, Peking University, Beijing, China

Y. Ban, G. Chen, A. Levin, J. Li, L. Li, Q. Li, Y. Mao, S. J. Qian, D. Wang

Tsinghua University, Beijing, China

Y. Wang

Universidad de Los Andes, Bogota, Colombia

C. Avila, A. Cabrera, C. A. Carrillo Montoya, L. F. Chaparro Sierra, C. Florez, C. F. González Hernández,

M. A. Segura Delgado

Faculty of Electrical Engineering, Mechanical Engineering and Naval Architecture, University of Split, Split, Croatia B. Courbon, N. Godinovic, D. Lelas, I. Puljak, T. Sculac

Faculty of Science, University of Split, Split, Croatia

Z. Antunovic, M. Kovac

Institute Rudjer Boskovic, Zagreb, Croatia

V. Brigljevic, D. Ferencek, K. Kadija, B. Mesic, M. Roguljic, A. Starodumov ${ }^{7}$, T. Susa

University of Cyprus, Nicosia, Cyprus

M. W. Ather, A. Attikis, M. Kolosova, G. Mavromanolakis, J. Mousa, C. Nicolaou, F. Ptochos, P. A. Razis, H. Rykaczewski

Charles University, Prague, Czech Republic

M. Finger ${ }^{8}$, M. Finger Jr. ${ }^{8}$

Escuela Politecnica Nacional, Quito, Ecuador

E. Ayala

Universidad San Francisco de Quito, Quito, Ecuador

E. Carrera Jarrin

Academy of Scientific Research and Technology of the Arab Republic of Egypt, Egyptian Network of High Energy Physics, Cairo, Egypt

A. Mahrous ${ }^{9}$, Y. Mohammed ${ }^{10}$, E. Salama ${ }^{11,12}$

National Institute of Chemical Physics and Biophysics, Tallinn, Estonia

S. Bhowmik, A. Carvalho Antunes De Oliveira, R. K. Dewanjee, K. Ehataht, M. Kadastik, M. Raidal, C. Veelken

Department of Physics, University of Helsinki, Helsinki, Finland

P. Eerola, H. Kirschenmann, J. Pekkanen, M. Voutilainen

Helsinki Institute of Physics, Helsinki, Finland

J. Havukainen, J. K. Heikkilä, T. Järvinen, V. Karimäki, R. Kinnunen, T. Lampén, K. Lassila-Perini, S. Laurila, S. Lehti, T. Lindén, P. Luukka, T. Mäenpää, H. Siikonen, E. Tuominen, J. Tuominiemi

Lappeenranta University of Technology, Lappeenranta, Finland

T. Tuuva

IRFU, CEA, Université Paris-Saclay, Gif-sur-Yvette, France

M. Besancon, F. Couderc, M. Dejardin, D. Denegri, J. L. Faure, F. Ferri, S. Ganjour, A. Givernaud, P. Gras,

G. Hamel de Monchenault, P. Jarry, C. Leloup, E. Locci, J. Malcles, G. Negro, J. Rander, A. Rosowsky, M. Ö. Sahin,

M. Titov

Laboratoire Leprince-Ringuet, Ecole polytechnique, CNRS/IN2P3, Université Paris-Saclay, Palaiseau, France A. Abdulsalam ${ }^{13}$, C. Amendola, I. Antropov, F. Beaudette, P. Busson, C. Charlot, R. Granier de Cassagnac, I. Kucher, A. Lobanov, J. Martin Blanco, C. Martin Perez, M. Nguyen, C. Ochando, G. Ortona, P. Paganini, J. Rembser, R. Salerno, J. B. Sauvan, Y. Sirois, A. G. Stahl Leiton, A. Zabi, A. Zghiche 
Université de Strasbourg, CNRS, IPHC UMR 7178, Strasbourg, France

J.-L. Agram ${ }^{14}$, J. Andrea, D. Bloch, J.-M. Brom, E. C. Chabert, V. Cherepanov, C. Collard, E. Conte ${ }^{14}$, J.-C. Fontaine ${ }^{14}$,

D. Gelé, U. Goerlach, M. Jansová, A.-C. Le Bihan, N. Tonon, P. Van Hove

Centre de Calcul de l'Institut National de Physique Nucleaire et de Physique des Particules, CNRS/IN2P3, Villeurbanne, France

S. Gadrat

CNRS-IN2P3, Institut de Physique Nucléaire de Lyon, Université de Lyon, Université Claude Bernard Lyon 1, Villeurbanne, France

S. Beauceron, C. Bernet, G. Boudoul, N. Chanon, R. Chierici, D. Contardo, P. Depasse, H. El Mamouni, J. Fay, L. Finco, S. Gascon, M. Gouzevitch, G. Grenier, B. Ille, F. Lagarde, I. B. Laktineh, H. Lattaud, M. Lethuillier, L. Mirabito,

S. Perries, A. Popov ${ }^{15}$, V. Sordini, G. Touquet, M. Vander Donckt, S. Viret

\section{Georgian Technical University, Tbilisi, Georgia}

T. Toriashvili ${ }^{16}$

Tbilisi State University, Tbilisi, Georgia

Z. Tsamalaidze 8

RWTH Aachen University, I. Physikalisches Institut, Aachen, Germany

C. Autermann, L. Feld, M. K. Kiesel, K. Klein, M. Lipinski, M. Preuten, M. P. Rauch, C. Schomakers, J. Schulz,

M. Teroerde, B. Wittmer

RWTH Aachen University, III. Physikalisches Institut A, Aachen, Germany

A. Albert, D. Duchardt, M. Erdmann, S. Erdweg, T. Esch, R. Fischer, S. Ghosh, A. Güth, T. Hebbeker, C. Heidemann, K. Hoepfner, H. Keller, L. Mastrolorenzo, M. Merschmeyer, A. Meyer, P. Millet, S. Mukherjee, T. Pook, M. Radziej, H. Reithler, M. Rieger, A. Schmidt, D. Teyssier, S. Thüer

RWTH Aachen University, III. Physikalisches Institut B, Aachen, Germany

G. Flügge, O. Hlushchenko, T. Kress, T. Müller, A. Nehrkorn, A. Nowack, C. Pistone, O. Pooth, D. Roy, H. Sert, A. Stahl ${ }^{17}$

\section{Deutsches Elektronen-Synchrotron, Hamburg, Germany}

M. Aldaya Martin, T. Arndt, C. Asawatangtrakuldee, I. Babounikau, K. Beernaert, O. Behnke, U. Behrens,

A. Bermúdez Martínez, D. Bertsche, A. A. Bin Anuar, K. Borras ${ }^{18}$, V. Botta, A. Campbell, P. Connor,

C. Contreras-Campana, V. Danilov, A. De Wit, M. M. Defranchis, C. Diez Pardos, D. Domínguez Damiani, G. Eckerlin,

T. Eichhorn, A. Elwood, E. Eren, E. Gallo ${ }^{19}$, A. Geiser, J. M. Grados Luyando, A. Grohsjean, M. Guthoff, M. Haranko,

A. Harb, H. Jung, M. Kasemann, J. Keaveney, C. Kleinwort, J. Knolle, D. Krücker, W. Lange, A. Lelek, T. Lenz,

J. Leonard, K. Lipka, W. Lohmann ${ }^{20}$, R. Mankel, I.-A. Melzer-Pellmann, A. B. Meyer, M. Meyer, M. Missiroli, J. Mnich,

V. Myronenko, S. K. Pflitsch, D. Pitzl, A. Raspereza, P. Saxena, P. Schütze, C. Schwanenberger, R. Shevchenko, A. Singh,

H. Tholen, O. Turkot, A. Vagnerini, M. Van De Klundert, G. P. Van Onsem, R. Walsh, Y. Wen, K. Wichmann, C. Wissing,

O. Zenaiev

\section{University of Hamburg, Hamburg, Germany}

R. Aggleton, S. Bein, L. Benato, A. Benecke, V. Blobel, T. Dreyer, A. Ebrahimi, E. Garutti, D. Gonzalez, P. Gunnellini, J. Haller, A. Hinzmann, A. Karavdina, G. Kasieczka, R. Klanner, R. Kogler, N. Kovalchuk, S. Kurz, V. Kutzner, J. Lange, D. Marconi, J. Multhaup, M. Niedziela, C. E. N. Niemeyer, D. Nowatschin, A. Perieanu, A. Reimers, O. Rieger, C. Scharf, P. Schleper, S. Schumann, J. Schwandt, J. Sonneveld, H. Stadie, G. Steinbrück, F. M. Stober, M. Stöver, B. Vormwald, I. Zoi

\section{Karlsruher Institut fuer Technologie, Karlsruhe, Germany}

M. Akbiyik, C. Barth, M. Baselga, S. Baur, E. Butz, R. Caspart, T. Chwalek, F. Colombo, W. De Boer, A. Dierlamm, K. El Morabit, N. Faltermann, B. Freund, M. Giffels, M. A. Harrendorf, F. Hartmann ${ }^{17}$, S. M. Heindl, U. Husemann, I. Katkov ${ }^{15}$, S. Kudella, S. Mitra, M. U. Mozer, Th. Müller, M. Musich, M. Plagge, G. Quast, K. Rabbertz, M. Schröder, I. Shvetsov, H. J. Simonis, R. Ulrich, S. Wayand, M. Weber, T. Weiler, C. Wöhrmann, R. Wolf

Institute of Nuclear and Particle Physics (INPP), NCSR Demokritos, Aghia Paraskevi, Greece

G. Anagnostou, G. Daskalakis, T. Geralis, A. Kyriakis, D. Loukas, G. Paspalaki 
National and Kapodistrian University of Athens, Athens, Greece

A. Agapitos, G. Karathanasis, P. Kontaxakis, A. Panagiotou, I. Papavergou, N. Saoulidou, E. Tziaferi, K. Vellidis

National Technical University of Athens, Athens, Greece

K. Kousouris, I. Papakrivopoulos, G. Tsipolitis

University of Ioánnina, Ioánnina, Greece

I. Evangelou, C. Foudas, P. Gianneios, P. Katsoulis, P. Kokkas, S. Mallios, N. Manthos, I. Papadopoulos, E. Paradas,

J. Strologas, F. A. Triantis, D. Tsitsonis

MTA-ELTE Lendület CMS Particle and Nuclear Physics Group, Eötvös Loránd University, Budapest, Hungary

M. Bartók ${ }^{21}$, M. Csanad, N. Filipovic, P. Major, M. I. Nagy, G. Pasztor, O. Surányi, G. I. Veres

Wigner Research Centre for Physics, Budapest, Hungary

G. Bencze, C. Hajdu, D. Horvath ${ }^{22}$, Á Hunyadi, F. Sikler, T. Á Vámi, V. Veszpremi, G. Vesztergombi ${ }^{\dagger}$

Institute of Nuclear Research ATOMKI, Debrecen, Hungary

N. Beni, S. Czellar, J. Karancsi2 ${ }^{21}$, A. Makovec, J. Molnar, Z. Szillasi

Institute of Physics, University of Debrecen, Debrecen, Hungary

P. Raics, Z. L. Trocsanyi, B. Ujvari

Indian Institute of Science (IISc), Bangalore, India

S. Choudhury, J. R. Komaragiri, P. C. Tiwari

National Institute of Science Education and Research, HBNI, Bhubaneswar, India

S. Bahinipati ${ }^{24}$, C. Kar, P. Mal, K. Mandal, A. Nayak ${ }^{25}$, S. Roy Chowdhury, D. K. Sahoo ${ }^{24}$, S. K. Swain

Panjab University, Chandigarh, India

S. Bansal, S. B. Beri, V. Bhatnagar, S. Chauhan, R. Chawla, N. Dhingra, S. K. Gill, R. Gupta, A. Kaur, M. Kaur,

P. Kumari, M. Lohan, M. Meena, A. Mehta, K. Sandeep, S. Sharma, J. B. Singh, A. K. Virdi, G. Walia

University of Delhi, Delhi, India

A. Bhardwaj, B. C. Choudhary, R. B. Garg, M. Gola, S. Keshri, Ashok Kumar, S. Malhotra, M. Naimuddin, P. Priyanka,

K. Ranjan, Aashaq Shah, R. Sharma

Saha Institute of Nuclear Physics, HBNI, Kolkata, India

R. Bhardwaj ${ }^{26}$, M. Bharti ${ }^{26}$, R. Bhattacharya, S. Bhattacharya, U. Bhawandeep ${ }^{26}$, D. Bhowmik, S. Dey, S. Dutt ${ }^{26}$, S. Dutta,

S. Ghosh, K. Mondal, S. Nandan, A. Purohit, P. K. Rout, A. Roy, G. Saha, S. Sarkar, M. Sharan, B. Singh ${ }^{26}$, S. Thakur ${ }^{26}$

Indian Institute of Technology Madras, Madras, India

P. K. Behera, A. Muhammad

Bhabha Atomic Research Centre, Mumbai, India

R. Chudasama, D. Dutta, V. Jha, V. Kumar, D. K. Mishra, P. K. Netrakanti, L. M. Pant, P. Shukla, P. Suggisetti

Tata Institute of Fundamental Research-A, Mumbai, India

T. Aziz, M. A. Bhat, S. Dugad, G. B. Mohanty, N. Sur, RavindraKumar Verma

Tata Institute of Fundamental Research-B, Mumbai, India

S. Banerjee, S. Bhattacharya, S. Chatterjee, P. Das, M. Guchait, Sa. Jain, S. Karmakar, S. Kumar, M. Maity ${ }^{27}$,

G. Majumder, K. Mazumdar, N. Sahoo, T. Sarkar ${ }^{27}$

Indian Institute of Science Education and Research (IISER), Pune, India

S. Chauhan, S. Dube, V. Hegde, A. Kapoor, K. Kothekar, S. Pandey, A. Rane, A. Rastogi, S. Sharma 
Institute for Research in Fundamental Sciences (IPM), Tehran, Iran

S. Chenarani ${ }^{28}$, E. Eskandari Tadavani, S. M. Etesami ${ }^{28}$, M. Khakzad, M. Mohammadi Najafabadi, M. Naseri,

F. Rezaei Hosseinabadi, B. Safarzadeh ${ }^{29}$, M. Zeinali

University College Dublin, Dublin, Ireland

M. Felcini, M. Grunewald

INFN Sezione di Bari ${ }^{a}$, Università di Bari ${ }^{b}$, Politecnico di Bari ${ }^{c}$, Bari, Italy

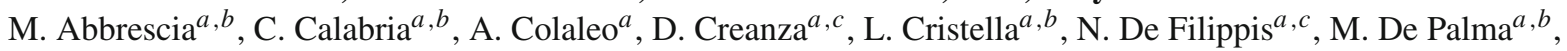

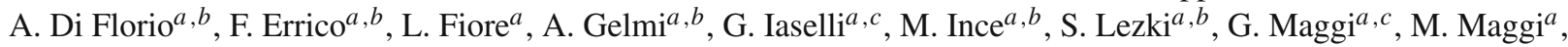

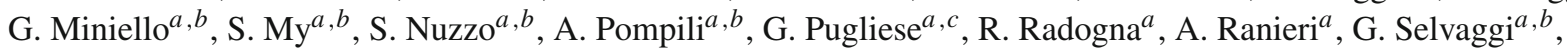

A. Sharma ${ }^{a}$, L. Silvestris ${ }^{a}$, R. Venditti ${ }^{a}$, P. Verwilligen ${ }^{a}$

INFN Sezione di Bologna ${ }^{a}$, Università di Bologna ${ }^{b}$, Bologna, Italy

G. Abbiendi ${ }^{a}$, C. Battilana ${ }^{a, b}$, D. Bonacorsi ${ }^{a, b}$, L. Borgonovi $^{a, b}$, S. Braibant-Giacomelli ${ }^{a, b}$, R. Campanini $^{a, b}$,

P. Capiluppi ${ }^{a, b}$, A. Castro ${ }^{a, b}$, F. R. Cavallo ${ }^{a}$, S. S. Chhibra ${ }^{a, b}$, G. Codispoti ${ }^{a, b}$, M. Cuffiani ${ }^{a, b}$, G. M. Dallavalle ${ }^{a}$,

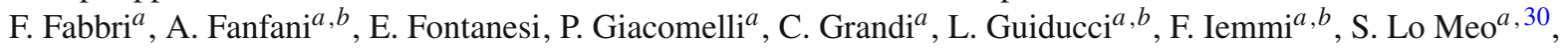

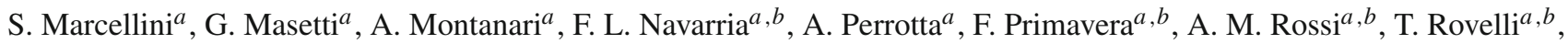

G. P. Siroli ${ }^{a, b}$, N. Tosi ${ }^{a}$

INFN Sezione di Catania ${ }^{a}$, Università di Catania ${ }^{b}$, Catania, Italy

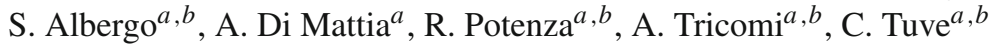

INFN Sezione di Firenze ${ }^{a}$, Università di Firenze ${ }^{b}$, Firenze, Italy

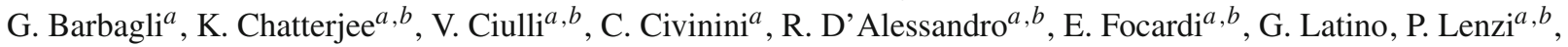

M. Meschini ${ }^{a}$, S. Paoletti $^{a}$, L. Russo $^{a}, 31$, G. Sguazzoni $^{a}$, D. Strom $^{a}$, L. Viliani $^{a}$

INFN Laboratori Nazionali di Frascati, Frascati, Italy

L. Benussi, S. Bianco, F. Fabbri, D. Piccolo

INFN Sezione di Genova ${ }^{a}$, Università di Genova ${ }^{b}$, Genova, Italy

F. Ferro ${ }^{a}$, R. Mulargia ${ }^{a, b}$, E. Robutti ${ }^{a}$, S. Tosi ${ }^{a, b}$

INFN Sezione di Milano-Bicocca ${ }^{a}$, Università di Milano-Bicocca ${ }^{b}$, Milano, Italy

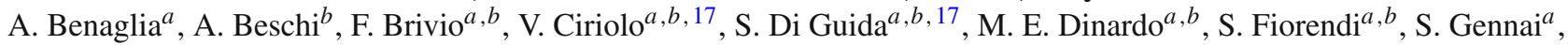

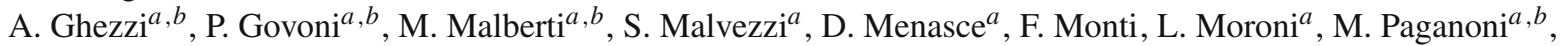

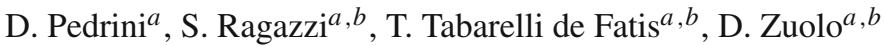

INFN Sezione di Napoli ${ }^{a}$, Università di Napoli 'Federico II' ${ }^{b}$, Napoli, Italy, Università della Basilicata ${ }^{c}$, Potenza, Italy, Università G. Marconi ${ }^{d}$, Roma, Italy

S. Buontempo ${ }^{a}$, N. Cavallo ${ }^{a, c}$, A. De Iorio ${ }^{a, b}$, A. Di Crescenzo ${ }^{a, b}$, F. Fabozzi $^{a, c}$, F. Fienga $^{a}$, G. Galati ${ }^{a}$, A. O. M. Iorio ${ }^{a, b}$,

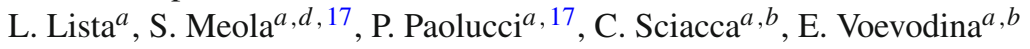

INFN Sezione di Padova ${ }^{a}$, Università di Padova ${ }^{b}$, Padova, Italy, Università di Trento $^{c}$, Trento, Italy

P. Azzi ${ }^{a}$, N. Bacchetta ${ }^{a}$, D. Bisello ${ }^{a, b}$, A. Boletti ${ }^{a, b}$, A. Bragagnolo, R. Carlin ${ }^{a, b}$, P. Checchia $^{a}$, M. Dall'Osso ${ }^{a, b}$,

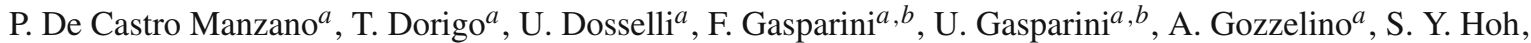

S. Lacaprara ${ }^{a}$, P. Lujan, M. Margoni ${ }^{a, b}$, A. T. Meneguzzo ${ }^{a, b}$, J. Pazzini $^{a, b}$, M. Presilla ${ }^{b}$, P. Ronchese ${ }^{a, b}$, R. Rossin ${ }^{a, b}$,

F. Simonetto ${ }^{a, b}$, A. Tiko, E. Torassa ${ }^{a}$, M. Tosi ${ }^{a, b}$, M. Zanetti ${ }^{a, b}$, P. Zotto ${ }^{a, b}$, G. Zumerle $^{a, b}$

INFN Sezione di Pavia ${ }^{a}$, Università di Pavia ${ }^{b}$, Pavia, Italy

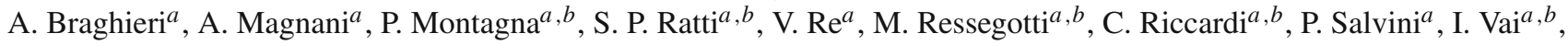
P. Vitulo ${ }^{a, b}$

INFN Sezione di Perugia ${ }^{a}$, Università di Perugia ${ }^{b}$, Perugia, Italy

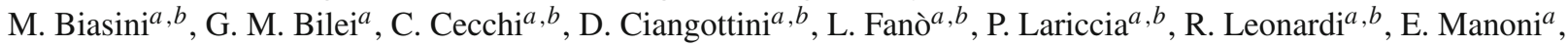
G. Mantovani ${ }^{a, b}$, V. Mariani ${ }^{a, b}$, M. Menichelli ${ }^{a}$, A. Rossi ${ }^{a, b}$, A. Santocchia ${ }^{a}, b$, D. Spiga ${ }^{a}$ 
INFN Sezione di Pisa ${ }^{a}$, Università di Pisa ${ }^{b}$, Scuola Normale Superiore di Pisa ${ }^{c}$, Pisa, Italy

K. Androsov ${ }^{a}$, P. Azzurri ${ }^{a}$, G. Bagliesi ${ }^{a}$, L. Bianchini $^{a}$, T. Boccali ${ }^{a}$, L. Borrello, R. Castaldi ${ }^{a}$, M. A. Ciocci ${ }^{a}, b$,

R. Dell'Orso ${ }^{a}$, G. Fedi ${ }^{a}$, F. Fiori ${ }^{a, c}$, L. Giannini ${ }^{a, c}$, A. Giassi ${ }^{a}$, M. T. Grippo ${ }^{a}$, F. Ligabue ${ }^{a, c}$, E. Manca $^{a, c}$, G. Mandorli $^{a, c}$,

A. Messineo ${ }^{a, b}$, F. Palla ${ }^{a}$, A. Rizzi ${ }^{a, b}$, G. Rolandi ${ }^{32}$, P. Spagnolo ${ }^{a}$, R. Tenchini ${ }^{a}$, G. Tonelli ${ }^{a}, b$, A. Venturi ${ }^{a}$, P. G. Verdini ${ }^{a}$

INFN Sezione di Roma ${ }^{a}$, Sapienza Università di Roma ${ }^{b}$, Rome, Italy

L. Barone ${ }^{a, b}$, F. Cavallari ${ }^{a}$, M. Cipriani ${ }^{a, b}$, D. Del Re ${ }^{a, b}$, E. Di Marco ${ }^{a, b}$, M. Diemoz ${ }^{a}$, S. Gelli ${ }^{a, b}$, E. Longo $^{a, b}$,

B. Marzocchi ${ }^{a, b}$, P. Meridiani ${ }^{a}$, G. Organtini ${ }^{a}, b$, F. Pandolfi ${ }^{a}$, R. Paramatti ${ }^{a}, b$, F. Preiato ${ }^{a, b}$, S. Rahatlou ${ }^{a, b}$, C. Rovelli $^{a}$,

F. Santanastasio ${ }^{a, b}$

INFN Sezione di Torino ${ }^{a}$, Università di Torino ${ }^{b}$, Torino, Italy, Università del Piemonte Orientale ${ }^{c}$, Novara, Italy

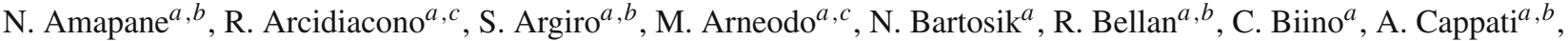

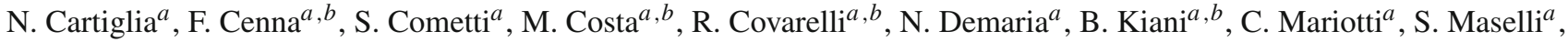

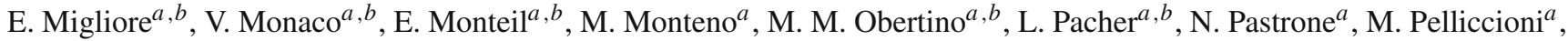

G. L. Pinna Angioni ${ }^{a, b}$, A. Romero ${ }^{a, b}$, M. Ruspa ${ }^{a, c}$, R. Sacchi ${ }^{a}, b$, R. Salvatico ${ }^{a, b}$, K. Shchelina ${ }^{a, b}$, V. Sola ${ }^{a}$, A. Solano ${ }^{a, b}$,

D. Soldi ${ }^{a}, b$ A. Staiano ${ }^{a}$

INFN Sezione di Trieste ${ }^{a}$, Università di Trieste ${ }^{b}$, Trieste, Italy

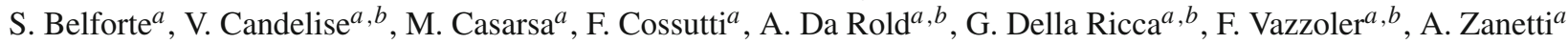

\section{Kyungpook National University, Daegu, Korea}

D. H. Kim, G. N. Kim, M. S. Kim, J. Lee, S. Lee, S. W. Lee, C. S. Moon, Y. D. Oh, S. I. Pak, S. Sekmen, D. C. Son,

Y. C. Yang

Chonnam National University, Institute for Universe and Elementary Particles, Kwangju, Korea

H. Kim, D. H. Moon, G. Oh

Hanyang University, Seoul, Korea

B. Francois, J. Goh ${ }^{33}$, T. J. Kim

Korea University, Seoul, Korea

S. Cho, S. Choi, Y. Go, D. Gyun, S. Ha, B. Hong, Y. Jo, K. Lee, K. S. Lee, S. Lee, J. Lim, S. K. Park, Y. Roh

Sejong University, Seoul, Korea

H. S. Kim

Seoul National University, Seoul, Korea

J. Almond, J. Kim, J. S. Kim, H. Lee, K. Lee, K. Nam, S. B. Oh, B. C. Radburn-Smith, S. h. Seo, U. K. Yang, H. D. Yoo,

G. B. Yu

University of Seoul, Seoul, Korea

D. Jeon, H. Kim, J. H. Kim, J. S. H. Lee, I. C. Park

\section{Sungkyunkwan University, Suwon, Korea}

Y. Choi, C. Hwang, J. Lee, I. Yu

Vilnius University, Vilnius, Lithuania

V. Dudenas, A. Juodagalvis, J. Vaitkus

National Centre for Particle Physics, Universiti Malaya, Kuala Lumpur, Malaysia

Z. A. Ibrahim, M. A. B. Md Ali ${ }^{34}$, F. Mohamad Idris ${ }^{35}$, W. A. T. Wan Abdullah, M. N. Yusli, Z. Zolkapli

Universidad de Sonora (UNISON), Hermosillo, Mexico

J. F. Benitez, A. Castaneda Hernandez, J. A. Murillo Quijada

Centro de Investigacion y de Estudios Avanzados del IPN, Mexico City, Mexico

H. Castilla-Valdez, E. De La Cruz-Burelo, M. C. Duran-Osuna, I. Heredia-De La Cruz ${ }^{36}$, R. Lopez-Fernandez,

J. Mejia Guisao, R. I. Rabadan-Trejo, M. Ramirez-Garcia, G. Ramirez-Sanchez, R. Reyes-Almanza, A. Sanchez-Hernandez 
Universidad Iberoamericana, Mexico City, Mexico

S. Carrillo Moreno, C. Oropeza Barrera, F. Vazquez Valencia

Benemerita Universidad Autonoma de Puebla, Puebla, Mexico

J. Eysermans, I. Pedraza, H. A. Salazar Ibarguen, C. Uribe Estrada

Universidad Autónoma de San Luis Potosí, San Luis Potosí, Mexico

A. Morelos Pineda

University of Auckland, Auckland, New Zealand

D. Krofcheck

University of Canterbury, Christchurch, New Zealand

S. Bheesette, P. H. Butler

National Centre for Physics, Quaid-I-Azam University, Islamabad, Pakistan

A. Ahmad, M. Ahmad, M. I. Asghar, Q. Hassan, H. R. Hoorani, W. A. Khan, A. Saddique, M. A. Shah, M. Shoaib, M. Waqas

National Centre for Nuclear Research, Swierk, Poland

H. Bialkowska, M. Bluj, B. Boimska, T. Frueboes, M. Górski, M. Kazana, M. Szleper, P. Traczyk, P. Zalewski

Institute of Experimental Physics, Faculty of Physics, University of Warsaw, Warsaw, Poland

K. Bunkowski, A. Byszuk ${ }^{37}$, K. Doroba, A. Kalinowski, M. Konecki, J. Krolikowski, M. Misiura, M. Olszewski,

A. Pyskir, M. Walczak

Laboratório de Instrumentação e Física Experimental de Partículas, Lisboa, Portugal

M. Araujo, P. Bargassa, C. Beirão Da Cruz E Silva, A. Di Francesco, P. Faccioli, B. Galinhas, M. Gallinaro, J. Hollar,

N. Leonardo, J. Seixas, G. Strong, O. Toldaiev, J. Varela

Joint Institute for Nuclear Research, Dubna, Russia

S. Afanasiev, P. Bunin, M. Gavrilenko, I. Golutvin, I. Gorbunov, A. Kamenev, V. Karjavine, A. Lanev, A. Malakhov,

V. Matveev ${ }^{38,39}$, P. Moisenz, V. Palichik, V. Perelygin, S. Shmatov, S. Shulha, N. Skatchkov, V. Smirnov, N. Voytishin,

A. Zarubin

Petersburg Nuclear Physics Institute, Gatchina (St. Petersburg), Russia

V. Golovtsov, Y. Ivanov, V. Kim ${ }^{40}$, E. Kuznetsova ${ }^{41}$, P. Levchenko, V. Murzin, V. Oreshkin, I. Smirnov, D. Sosnov,

V. Sulimov, L. Uvarov, S. Vavilov, A. Vorobyev

Institute for Nuclear Research, Moscow, Russia

Yu. Andreev, A. Dermenev, S. Gninenko, N. Golubev, A. Karneyeu, M. Kirsanov, N. Krasnikov, A. Pashenkov,

A. Shabanov, D. Tlisov, A. Toropin

Institute for Theoretical and Experimental Physics, Moscow, Russia

V. Epshteyn, V. Gavrilov, N. Lychkovskaya, V. Popov, I. Pozdnyakov, G. Safronov, A. Spiridonov, A. Stepennov,

V. Stolin, M. Toms, E. Vlasov, A. Zhokin

Moscow Institute of Physics and Technology, Moscow, Russia

T. Aushev

National Research Nuclear University 'Moscow Engineering Physics Institute' (MEPhI), Moscow, Russia

R. Chistov ${ }^{42}$, M. Danilov ${ }^{42}$, S. Polikarpov ${ }^{42}$, E. Tarkovskii

P.N. Lebedev Physical Institute, Moscow, Russia

V. Andreev, M. Azarkin, I. Dremin ${ }^{39}$, M. Kirakosyan, A. Terkulov

Skobeltsyn Institute of Nuclear Physics, Lomonosov Moscow State University, Moscow, Russia

A. Baskakov, A. Belyaev, E. Boos, M. Dubinin ${ }^{43}$, L. Dudko, A. Ershov, A. Gribushin, V. Klyukhin, O. Kodolova, I. Lokhtin, I. Miagkov, S. Obraztsov, S. Petrushanko, V. Savrin, A. Snigirev 
Novosibirsk State University (NSU), Novosibirsk, Russia

A. Barnyakov ${ }^{44}$, V. Blinov ${ }^{44}$, T. Dimova ${ }^{44}$, L. Kardapoltsev ${ }^{44}$, Y. Skovpen ${ }^{44}$

Institute for High Energy Physics of National Research Centre 'Kurchatov Institute', Protvino, Russia

I. Azhgirey, I. Bayshev, S. Bitioukov, V. Kachanov, A. Kalinin, D. Konstantinov, P. Mandrik, V. Petrov, R. Ryutin,

S. Slabospitskii, A. Sobol, S. Troshin, N. Tyurin, A. Uzunian, A. Volkov

National Research Tomsk Polytechnic University, Tomsk, Russia

A. Babaev, S. Baidali, V. Okhotnikov

Faculty of Physics and VINCA Institute of Nuclear Sciences, University of Belgrade, Belgrade, Serbia

P. Adzic ${ }^{45}$, P. Cirkovic, D. Devetak, M. Dordevic, J. Milosevic

Centro de Investigaciones Energéticas Medioambientales y Tecnológicas (CIEMAT), Madrid, Spain

J. Alcaraz Maestre, A. Álvarez Fernández, I. Bachiller, M. Barrio Luna, J. A. Brochero Cifuentes, M. Cerrada, N. Colino,

B. De La Cruz, A. Delgado Peris, C. Fernandez Bedoya, J. P. Fernández Ramos, J. Flix, M. C. Fouz, O. Gonzalez Lopez,

S. Goy Lopez, J. M. Hernandez, M. I. Josa, D. Moran, A. Pérez-Calero Yzquierdo, J. Puerta Pelayo, I. Redondo,

L. Romero, S. Sánchez Navas, M. S. Soares, A. Triossi

Universidad Autónoma de Madrid, Madrid, Spain

C. Albajar, J. F. de Trocóniz

Universidad de Oviedo, Oviedo, Spain

J. Cuevas, C. Erice, J. Fernandez Menendez, S. Folgueras, I. Gonzalez Caballero, J. R. González Fernández,

E. Palencia Cortezon, V. Rodríguez Bouza, S. Sanchez Cruz, J. M. Vizan Garcia

Instituto de Física de Cantabria (IFCA), CSIC-Universidad de Cantabria, Santander, Spain

I. J. Cabrillo, A. Calderon, B. Chazin Quero, J. Duarte Campderros, M. Fernandez, P. J. Fernández Manteca,

A. García Alonso, J. Garcia-Ferrero, G. Gomez, A. Lopez Virto, J. Marco, C. Martinez Rivero, P. Martinez Ruiz del Arbol,

F. Matorras, J. Piedra Gomez, C. Prieels, T. Rodrigo, A. Ruiz-Jimeno, L. Scodellaro, N. Trevisani, I. Vila,

R. Vilar Cortabitarte

Department of Physics, University of Ruhuna, Matara, Sri Lanka

N. Wickramage

CERN, European Organization for Nuclear Research, Geneva, Switzerland

D. Abbaneo, B. Akgun, E. Auffray, G. Auzinger, P. Baillon, A. H. Ball, D. Barney, J. Bendavid, M. Bianco, A. Bocci,

C. Botta, E. Brondolin, T. Camporesi, M. Cepeda, G. Cerminara, E. Chapon, Y. Chen, G. Cucciati, D. d'Enterria,

A. Dabrowski, N. Daci, V. Daponte, A. David, A. De Roeck, N. Deelen, M. Dobson, M. Dünser, N. Dupont,

A. Elliott-Peisert, P. Everaerts, F. Fallavollita ${ }^{46}$, D. Fasanella, G. Franzoni, J. Fulcher, W. Funk, D. Gigi, A. Gilbert,

K. Gill, F. Glege, M. Gruchala, M. Guilbaud, D. Gulhan, J. Hegeman, C. Heidegger, V. Innocente, A. Jafari, P. Janot,

O. Karacheban ${ }^{20}$, J. Kieseler, A. Kornmayer, M. Krammer ${ }^{1}$, C. Lange, P. Lecoq, C. Lourenço, L. Malgeri, M. Mannelli,

A. Massironi, F. Meijers, J. A. Merlin, S. Mersi, E. Meschi, P. Milenovic ${ }^{47}$, F. Moortgat, M. Mulders, J. Ngadiuba,

S. Nourbakhsh, S. Orfanelli, L. Orsini, F. Pantaleo ${ }^{17}$, L. Pape, E. Perez, M. Peruzzi, A. Petrilli, G. Petrucciani, A. Pfeiffer,

M. Pierini, F. M. Pitters, D. Rabady, A. Racz, T. Reis, M. Rovere, H. Sakulin, C. Schäfer, C. Schwick, M. Selvaggi,

A. Sharma, P. Silva, P. Sphicas ${ }^{48}$, A. Stakia, J. Steggemann, D. Treille, A. Tsirou, A. Vartak, V. Veckalns ${ }^{49}$, M. Verzetti,

W. D. Zeuner

Paul Scherrer Institut, Villigen, Switzerland

L. Caminada ${ }^{50}$, K. Deiters, W. Erdmann, R. Horisberger, Q. Ingram, H. C. Kaestli, D. Kotlinski, U. Langenegger, T. Rohe, S. A. Wiederkehr

ETH Zurich, Institute for Particle Physics and Astrophysics (IPA), Zurich, Switzerland M. Backhaus, L. Bäni, P. Berger, N. Chernyavskaya, G. Dissertori, M. Dittmar, M. Donegà, C. Dorfer,

T. A. Gómez Espinosa, C. Grab, D. Hits, T. Klijnsma, W. Lustermann, R. A. Manzoni, M. Marionneau, M. T. Meinhard,

F. Micheli, P. Musella, F. Nessi-Tedaldi, F. Pauss, G. Perrin, L. Perrozzi, S. Pigazzini, C. Reissel, D. Ruini,

D. A. Sanz Becerra, M. Schönenberger, L. Shchutska, V. R. Tavolaro, K. Theofilatos, M. L. Vesterbacka Olsson, R. Wallny,

D. H. Zhu 


\section{Universität Zürich, Zurich, Switzerland}

T. K. Aarrestad, C. Amsler ${ }^{51}$, D. Brzhechko, M. F. Canelli, A. De Cosa, R. Del Burgo, S. Donato, C. Galloni, T. Hreus,

B. Kilminster, S. Leontsinis, I. Neutelings, G. Rauco, P. Robmann, D. Salerno, K. Schweiger, C. Seitz, Y. Takahashi,

A. Zucchetta

National Central University, Chung-Li, Taiwan

T. H. Doan, R. Khurana, C. M. Kuo, W. Lin, A. Pozdnyakov, S. S. Yu

National Taiwan University (NTU), Taipei, Taiwan

P. Chang, Y. Chao, K. F. Chen, P. H. Chen, W.-S. Hou, Y. F. Liu, R.-S. Lu, E. Paganis, A. Psallidas, A. Steen

Chulalongkorn University, Faculty of Science, Department of Physics, Bangkok, Thailand

B. Asavapibhop, N. Srimanobhas, N. Suwonjandee

Çukurova University, Physics Department, Science and Art Faculty, Adana, Turkey

A. Bat, F. Boran, S. Cerci ${ }^{52}$, S. Damarseckin, Z. S. Demiroglu, F. Dolek, C. Dozen, E. Eskut, G. Gokbulut, Y. Guler,

E. Gurpinar, I. $\operatorname{Hos}^{53}$, C. Isik, E. E. Kangal ${ }^{54}$, O. Kara, A. Kayis Topaksu, U. Kiminsu, M. Oglakci, G. Onengut,

K. Ozdemir ${ }^{55}$, A. Polatoz, D. Sunar Cerci ${ }^{52}$, B. Tali ${ }^{52}$, U. G. Tok, S. Turkcapar, I. S. Zorbakir, C. Zorbilmez

Middle East Technical University, Physics Department, Ankara, Turkey

B. Isildak ${ }^{56}$, G. Karapinar ${ }^{57}$, M. Yalvac, M. Zeyrek

Bogazici University, Istanbul, Turkey

I. O. Atakisi, E. Gülmez, M. Kaya ${ }^{58}$, O. Kaya ${ }^{59}$, S. Ozkorucuklu ${ }^{60}$, S. Tekten, E. A. Yetkin ${ }^{61}$

Istanbul Technical University, Istanbul, Turkey

M. N. Agaras, A. Cakir, K. Cankocak, Y. Komurcu, S. Sen ${ }^{62}$

Institute for Scintillation Materials of National Academy of Science of Ukraine, Kharkov, Ukraine

B. Grynyov

National Scientific Center, Kharkov Institute of Physics and Technology, Kharkov, Ukraine

L. Levchuk

University of Bristol, Bristol, UK

F. Ball, J. J. Brooke, D. Burns, E. Clement, D. Cussans, O. Davignon, H. Flacher, J. Goldstein, G. P. Heath, H. F. Heath, L. Kreczko, D. M. Newbold ${ }^{63}$, S. Paramesvaran, B. Penning, T. Sakuma, D. Smith, V. J. Smith, J. Taylor, A. Titterton

\section{Rutherford Appleton Laboratory, Didcot, UK}

K. W. Bell, A. Belyaev ${ }^{64}$, C. Brew, R. M. Brown, D. Cieri, D. J. A. Cockerill, J. A. Coughlan, K. Harder, S. Harper, J. Linacre, K. Manolopoulos, E. Olaiya, D. Petyt, C. H. Shepherd-Themistocleous, A. Thea, I. R. Tomalin, T. Williams, W. J. Womersley

\section{Imperial College, London, UK}

R. Bainbridge, P. Bloch, J. Borg, S. Breeze, O. Buchmuller, A. Bundock, D. Colling, P. Dauncey, G. Davies, M. Della Negra, R. Di Maria, G. Hall, G. Iles, T. James, M. Komm, L. Lyons, A.-M. Magnan, S. Malik, A. Martelli, J. Nash ${ }^{65}$, A. Nikitenko ${ }^{7}$, V. Palladino, M. Pesaresi, D. M. Raymond, A. Richards, A. Rose, E. Scott, C. Seez, A. Shtipliyski, G. Singh, M. Stoye, T. Strebler, S. Summers, A. Tapper, K. Uchida, T. Virdee ${ }^{17}$, N. Wardle, D. Winterbottom, S. C. Zenz

Brunel University, Uxbridge, UK

J. E. Cole, P. R. Hobson, A. Khan, P. Kyberd, C. K. Mackay, A. Morton, I. D. Reid, L. Teodorescu, S. Zahid

Baylor University, Waco, USA

K. Call, J. Dittmann, K. Hatakeyama, H. Liu, C. Madrid, B. McMaster, N. Pastika, C. Smith

Catholic University of America, Washington, DC, USA

R. Bartek, A. Dominguez

The University of Alabama, Tuscaloosa, USA

A. Buccilli, S. I. Cooper, C. Henderson, P. Rumerio, C. West 


\section{Boston University, Boston, USA}

D. Arcaro, T. Bose, D. Gastler, S. Girgis, D. Pinna, D. Rankin, C. Richardson, J. Rohlf, L. Sulak, D. Zou

\section{Brown University, Providence, USA}

G. Benelli, X. Coubez, D. Cutts, M. Hadley, J. Hakala, U. Heintz, J. M. Hogan ${ }^{66}$, K. H. M. Kwok, E. Laird, G. Landsberg, J. Lee, Z. Mao, M. Narain, S. Sagir ${ }^{67}$, R. Syarif, E. Usai, D. Yu

University of California, Davis, Davis, USA

R. Band, C. Brainerd, R. Breedon, D. Burns, M. Calderon De La Barca Sanchez, M. Chertok, J. Conway, R. Conway, P. T. Cox, R. Erbacher, C. Flores, G. Funk, W. Ko, O. Kukral, R. Lander, M. Mulhearn, D. Pellett, J. Pilot, S. Shalhout, M. Shi, D. Stolp, D. Taylor, K. Tos, M. Tripathi, Z. Wang, F. Zhang

University of California, Los Angeles, USA

M. Bachtis, C. Bravo, R. Cousins, A. Dasgupta, A. Florent, J. Hauser, M. Ignatenko, N. Mccoll, S. Regnard, D. Saltzberg, C. Schnaible, V. Valuev

\section{University of California, Riverside, Riverside, USA}

E. Bouvier, K. Burt, R. Clare, J. W. Gary, S. M. A. Ghiasi Shirazi, G. Hanson, G. Karapostoli, E. Kennedy, F. Lacroix, O. R. Long, M. Olmedo Negrete, M. I. Paneva, W. Si, L. Wang, H. Wei, S. Wimpenny, B. R. Yates

\section{University of California, San Diego, La Jolla, USA}

J. G. Branson, P. Chang, S. Cittolin, M. Derdzinski, R. Gerosa, D. Gilbert, B. Hashemi, A. Holzner, D. Klein, G. Kole, V. Krutelyov, J. Letts, M. Masciovecchio, D. Olivito, S. Padhi, M. Pieri, V. Sharma, S. Simon, M. Tadel, J. Wood, F. Würthwein, A. Yagil, G. Zevi Della Porta

\section{Department of Physics, University of California, Santa Barbara, Santa Barbara, USA}

N. Amin, R. Bhandari, C. Campagnari, M. Citron, V. Dutta, M. Franco Sevilla, L. Gouskos, R. Heller, J. Incandela, H. Mei, A. Ovcharova, H. Qu, J. Richman, D. Stuart, I. Suarez, S. Wang, J. Yoo

\section{California Institute of Technology, Pasadena, USA}

D. Anderson, A. Bornheim, J. M. Lawhorn, N. Lu, H. B. Newman, T. Q. Nguyen, J. Pata, M. Spiropulu, J. R. Vlimant, R. Wilkinson, S. Xie, Z. Zhang, R. Y. Zhu

\section{Carnegie Mellon University, Pittsburgh, USA}

M. B. Andrews, T. Ferguson, T. Mudholkar, M. Paulini, M. Sun, I. Vorobiev, M. Weinberg

\section{University of Colorado Boulder, Boulder, USA}

J. P. Cumalat, W. T. Ford, F. Jensen, A. Johnson, E. MacDonald, T. Mulholland, R. Patel, A. Perloff, K. Stenson, K. A. Ulmer, S. R. Wagner

\section{Cornell University, Ithaca, USA}

J. Alexander, J. Chaves, Y. Cheng, J. Chu, A. Datta, K. Mcdermott, N. Mirman, J. R. Patterson, D. Quach, A. Rinkevicius, A. Ryd, L. Skinnari, L. Soffi, S. M. Tan, Z. Tao, J. Thom, J. Tucker, P. Wittich, M. Zientek

\section{Fermi National Accelerator Laboratory, Batavia, USA}

S. Abdullin, M. Albrow, M. Alyari, G. Apollinari, A. Apresyan, A. Apyan, S. Banerjee, L. A. T. Bauerdick, A. Beretvas, J. Berryhill, P. C. Bhat, K. Burkett, J. N. Butler, A. Canepa, G. B. Cerati, H. W. K. Cheung, F. Chlebana, M. Cremonesi, J. Duarte, V. D. Elvira, J. Freeman, Z. Gecse, E. Gottschalk, L. Gray, D. Green, S. Grünendahl, O. Gutsche, J. Hanlon, R. M. Harris, S. Hasegawa, J. Hirschauer, Z. Hu, B. Jayatilaka, S. Jindariani, M. Johnson, U. Joshi, B. Klima, M. J. Kortelainen, B. Kreis, S. Lammel, D. Lincoln, R. Lipton, M. Liu, T. Liu, J. Lykken, K. Maeshima, J. M. Marraffino, D. Mason, P. McBride, P. Merkel, S. Mrenna, S. Nahn, V. O’Dell, K. Pedro, C. Pena, O. Prokofyev, G. Rakness, F. Ravera, A. Reinsvold, L. Ristori, A. Savoy-Navarro ${ }^{68}$, B. Schneider, E. Sexton-Kennedy, A. Soha, W. J. Spalding, L. Spiegel, S. Stoynev, J. Strait, N. Strobbe, L. Taylor, S. Tkaczyk, N. V. Tran, L. Uplegger, E. W. Vaandering, C. Vernieri, M. Verzocchi, R. Vidal, M. Wang, H. A. Weber, A. Whitbeck 
University of Florida, Gainesville, USA

D. Acosta, P. Avery, P. Bortignon, D. Bourilkov, A. Brinkerhoff, L. Cadamuro, A. Carnes, D. Curry, R. D. Field,

S. V. Gleyzer, B. M. Joshi, J. Konigsberg, A. Korytov, K. H. Lo, P. Ma, K. Matchev, G. Mitselmakher, D. Rosenzweig,

K. Shi, D. Sperka, J. Wang, S. Wang, X. Zuo

Florida International University, Miami, USA

Y. R. Joshi, S. Linn

Florida State University, Tallahassee, USA

A. Ackert, T. Adams, A. Askew, S. Hagopian, V. Hagopian, K. F. Johnson, T. Kolberg, G. Martinez, T. Perry, H. Prosper, A. Saha, C. Schiber, R. Yohay

\section{Florida Institute of Technology, Melbourne, USA}

M. M. Baarmand, V. Bhopatkar, S. Colafranceschi, M. Hohlmann, D. Noonan, M. Rahmani, T. Roy, M. Saunders,

F. Yumiceva

\section{University of Illinois at Chicago (UIC), Chicago, USA}

M. R. Adams, L. Apanasevich, D. Berry, R. R. Betts, R. Cavanaugh, X. Chen, S. Dittmer, O. Evdokimov, C. E. Gerber,

D. A. Hangal, D. J. Hofman, K. Jung, J. Kamin, C. Mills, M. B. Tonjes, N. Varelas, H. Wang, X. Wang, Z. Wu, J. Zhang

The University of Iowa, Iowa City, USA

M. Alhusseini, B. Bilki ${ }^{69}$, W. Clarida, K. Dilsiz ${ }^{70}$, S. Durgut, R. P. Gandrajula, M. Haytmyradov, V. Khristenko,

J.-P. Merlo, A. Mestvirishvili, A. Moeller, J. Nachtman, H. Ogul ${ }^{71}$, Y. Onel, F. Ozok ${ }^{72}$, A. Penzo, C. Snyder, E. Tiras,

J. Wetzel

Johns Hopkins University, Baltimore, USA

B. Blumenfeld, A. Cocoros, N. Eminizer, D. Fehling, L. Feng, A. V. Gritsan, W. T. Hung, P. Maksimovic, J. Roskes,

U. Sarica, M. Swartz, M. Xiao

The University of Kansas, Lawrence, USA

A. Al-bataineh, P. Baringer, A. Bean, S. Boren, J. Bowen, A. Bylinkin, J. Castle, S. Khalil, A. Kropivnitskaya,

D. Majumder, W. Mcbrayer, M. Murray, C. Rogan, S. Sanders, E. Schmitz, J. D. Tapia Takaki, Q. Wang

Kansas State University, Manhattan, USA

S. Duric, A. Ivanov, K. Kaadze, D. Kim, Y. Maravin, D. R. Mendis, T. Mitchell, A. Modak, A. Mohammadi

\section{Lawrence Livermore National Laboratory, Livermore, USA}

F. Rebassoo, D. Wright

University of Maryland, College Park, USA

A. Baden, O. Baron, A. Belloni, S. C. Eno, Y. Feng, C. Ferraioli, N. J. Hadley, S. Jabeen, G. Y. Jeng, R. G. Kellogg,

J. Kunkle, A. C. Mignerey, S. Nabili, F. Ricci-Tam, M. Seidel, Y. H. Shin, A. Skuja, S. C. Tonwar, K. Wong

\section{Massachusetts Institute of Technology, Cambridge, USA}

D. Abercrombie, B. Allen, V. Azzolini, A. Baty, G. Bauer, R. Bi, S. Brandt, W. Busza, I. A. Cali, M. D’Alfonso,

Z. Demiragli, G. Gomez Ceballos, M. Goncharov, P. Harris, D. Hsu, M. Hu, Y. Iiyama, G. M. Innocenti, M. Klute,

D. Kovalskyi, Y.-J. Lee, P. D. Luckey, B. Maier, A. C. Marini, C. Mcginn, C. Mironov, S. Narayanan, X. Niu, C. Paus,

C. Roland, G. Roland, Z. Shi, G. S. F. Stephans, K. Sumorok, K. Tatar, D. Velicanu, J. Wang, T. W. Wang, B. Wyslouch

University of Minnesota, Minneapolis, USA

A. C. Benvenuti ${ }^{\dagger}$, R. M. Chatterjee, A. Evans, P. Hansen, J. Hiltbrand, Sh. Jain, S. Kalafut, M. Krohn, Y. Kubota, Z. Lesko, J. Mans, N. Ruckstuhl, R. Rusack, M. A. Wadud

University of Mississippi, Oxford, USA

J. G. Acosta, S. Oliveros 


\section{University of Nebraska-Lincoln, Lincoln, USA}

E. Avdeeva, K. Bloom, D. R. Claes, C. Fangmeier, F. Golf, R. Gonzalez Suarez, R. Kamalieddin, I. Kravchenko,

J. Monroy, J. E. Siado, G. R. Snow, B. Stieger

\section{State University of New York at Buffalo, Buffalo, USA}

A. Godshalk, C. Harrington, I. Iashvili, A. Kharchilava, C. Mclean, D. Nguyen, A. Parker, S. Rappoccio, B. Roozbahani

\section{Northeastern University, Boston, USA}

G. Alverson, E. Barberis, C. Freer, Y. Haddad, A. Hortiangtham, G. Madigan, D. M. Morse, T. Orimoto,

A. Tishelman-charny, T. Wamorkar, B. Wang, A. Wisecarver, D. Wood

\section{Northwestern University, Evanston, USA}

S. Bhattacharya, J. Bueghly, O. Charaf, T. Gunter, K. A. Hahn, N. Odell, M. H. Schmitt, K. Sung, M. Trovato, M. Velasco

\section{University of Notre Dame, Notre Dame, USA}

R. Bucci, N. Dev, M. Hildreth, K. Hurtado Anampa, C. Jessop, D. J. Karmgard, K. Lannon, W. Li, N. Loukas,

N. Marinelli, F. Meng, C. Mueller, Y. Musienko ${ }^{38}$, M. Planer, R. Ruchti, P. Siddireddy, G. Smith, S. Taroni, M. Wayne,

A. Wightman, M. Wolf, A. Woodard

The Ohio State University, Columbus, USA

J. Alimena, L. Antonelli, B. Bylsma, L. S. Durkin, S. Flowers, B. Francis, C. Hill, W. Ji, T. Y. Ling, W. Luo, B. L. Winer

\section{Princeton University, Princeton, USA}

S. Cooperstein, P. Elmer, J. Hardenbrook, N. Haubrich, S. Higginbotham, A. Kalogeropoulos, S. Kwan, D. Lange, M. T. Lucchini, J. Luo, D. Marlow, K. Mei, I. Ojalvo, J. Olsen, C. Palmer, P. Piroué, J. Salfeld-Nebgen, D. Stickland, C. Tully

\section{University of Puerto Rico, Mayaguez, USA}

S. Malik, S. Norberg

\section{Purdue University, West Lafayette, USA}

A. Barker, V. E. Barnes, S. Das, L. Gutay, M. Jones, A. W. Jung, A. Khatiwada, B. Mahakud, D. H. Miller, N. Neumeister,

C. C. Peng, S. Piperov, H. Qiu, J. F. Schulte, J. Sun, F. Wang, R. Xiao, W. Xie

\section{Purdue University Northwest, Hammond, USA}

T. Cheng, J. Dolen, N. Parashar

\section{Rice University, Houston, USA}

Z. Chen, K. M. Ecklund, S. Freed, F. J. M. Geurts, M. Kilpatrick, Arun Kumar, W. Li, B. P. Padley, R. Redjimi, J. Roberts, J. Rorie, W. Shi, Z. Tu, A. Zhang

\section{University of Rochester, Rochester, USA}

A. Bodek, P. de Barbaro, R. Demina, Y. T. Duh, J. L. Dulemba, C. Fallon, T. Ferbel, M. Galanti, A. Garcia-Bellido, J. Han, O. Hindrichs, A. Khukhunaishvili, E. Ranken, P. Tan, R. Taus

\section{Rutgers, The State University of New Jersey, Piscataway, USA}

B. Chiarito, J. P. Chou, Y. Gershtein, E. Halkiadakis, A. Hart, M. Heindl, E. Hughes, S. Kaplan,

R. Kunnawalkam Elayavalli, S. Kyriacou, I. Laflotte, A. Lath, R. Montalvo, K. Nash, M. Osherson, H. Saka, S. Salur,

S. Schnetzer, D. Sheffield, S. Somalwar, R. Stone, S. Thomas, P. Thomassen

\section{University of Tennessee, Knoxville, USA}

A. G. Delannoy, J. Heideman, G. Riley, S. Spanier

\section{Texas A \& M University, College Station, USA}

O. Bouhali 73 , A. Celik, M. Dalchenko, M. De Mattia, A. Delgado, S. Dildick, R. Eusebi, J. Gilmore, T. Huang,

T. Kamon ${ }^{74}$, S. Luo, D. Marley, R. Mueller, D. Overton, L. Perniè, D. Rathjens, A. Safonov

\section{Texas Tech University, Lubbock, USA}

N. Akchurin, J. Damgov, F. De Guio, P. R. Dudero, S. Kunori, K. Lamichhane, S. W. Lee, T. Mengke, S. Muthumuni, T. Peltola, S. Undleeb, I. Volobouev, Z. Wang 


\section{Vanderbilt University, Nashville, USA}

S. Greene, A. Gurrola, R. Janjam, W. Johns, C. Maguire, A. Melo, H. Ni, K. Padeken, F. Romeo, J. D. Ruiz Alvarez, P. Sheldon, S. Tuo, J. Velkovska, M. Verweij, Q. Xu

\section{University of Virginia, Charlottesville, USA}

M. W. Arenton, P. Barria, B. Cox, R. Hirosky, M. Joyce, A. Ledovskoy, H. Li, C. Neu, T. Sinthuprasith, Y. Wang,

E. Wolfe, F. Xia

\section{Wayne State University, Detroit, USA}

R. Harr, P. E. Karchin, N. Poudyal, J. Sturdy, P. Thapa, S. Zaleski

\section{University of Wisconsin, Madison, Madison, WI, USA}

J. Buchanan, C. Caillol, D. Carlsmith, S. Dasu, I. De Bruyn, L. Dodd, B. Gomber ${ }^{75}$, M. Grothe, M. Herndon, A. Hervé, U. Hussain, P. Klabbers, A. Lanaro, K. Long, R. Loveless, T. Ruggles, A. Savin, V. Sharma, N. Smith, W. H. Smith, N. Woods

\section{${ }^{\dagger}$ Deceased}

1: Also at Vienna University of Technology, Vienna, Austria

2: Also at IRFU, CEA, Université Paris-Saclay, Gif-sur-Yvette, France

3: Also at Universidade Estadual de Campinas, Campinas, Brazil

4: Also at Federal University of Rio Grande do Sul, Porto Alegre, Brazil

5: Also at Université Libre de Bruxelles, Bruxelles, Belgium

6: Also at University of Chinese Academy of Sciences, Beijing, China

7: Also at Institute for Theoretical and Experimental Physics, Moscow, Russia

8: Also at Joint Institute for Nuclear Research, Dubna, Russia

9: Now at Helwan University, Cairo, Egypt

10: Now at Fayoum University, El-Fayoum, Egypt

11: Also at British University in Egypt, Cairo, Egypt

12: Now at Ain Shams University, Cairo, Egypt

13: Also at Department of Physics, King Abdulaziz University, Jeddah, Saudi Arabia

14: Also at Université de Haute Alsace, Mulhouse, France

15: Also at Skobeltsyn Institute of Nuclear Physics, Lomonosov Moscow State University, Moscow, Russia

16: Also at Tbilisi State University, Tbilisi, Georgia

17: Also at CERN, European Organization for Nuclear Research, Geneva, Switzerland

18: Also at RWTH Aachen University, III. Physikalisches Institut A, Aachen, Germany

19: Also at University of Hamburg, Hamburg, Germany

20: Also at Brandenburg University of Technology, Cottbus, Germany

21: Also at Institute of Physics, University of Debrecen, Debrecen, Hungary

22: Also at Institute of Nuclear Research ATOMKI, Debrecen, Hungary

23: Also at MTA-ELTE Lendület CMS Particle and Nuclear Physics Group, Eötvös Loránd University, Budapest, Hungary

24: Also at Indian Institute of Technology Bhubaneswar, Bhubaneswar, India

25: Also at Institute of Physics, Bhubaneswar, India

26: Also at Shoolini University, Solan, India

27: Also at University of Visva-Bharati, Santiniketan, India

28: Also at Isfahan University of Technology, Isfahan, Iran

29: Also at Plasma Physics Research Center, Science and Research Branch, Islamic Azad University, Tehran, Iran

30: Also at Italian National Agency for New Technologies, Energy and Sustainable Economic Development, Bologna, Italy

31: Also at Università degli Studi di Siena, Siena, Italy

32: Also at Scuola Normale e Sezione dell'INFN, Pisa, Italy

33: Also at Kyung Hee University, Department of Physics, Seoul, Korea

34: Also at International Islamic University, of Malaysia, Kuala Lumpur, Malaysia

35: Also at Malaysian Nuclear Agency, MOSTI, Kajang, Malaysia

36: Also at Consejo Nacional de Ciencia y Tecnología, Mexico City, Mexico

37: Also at Warsaw University of Technology, Institute of Electronic Systems, Warsaw, Poland 
38: Also at Institute for Nuclear Research, Moscow, Russia

39: Now at National Research Nuclear University 'Moscow Engineering Physics Institute' (MEPhI), Moscow, Russia

40: Also at St. Petersburg State Polytechnical University, St. Petersburg, Russia

41: Also at University of Florida, Gainesville, USA

42: Also at P.N. Lebedev Physical Institute, Moscow, Russia

43: Also at California Institute of Technology, Pasadena, USA

44: Also at Budker Institute of Nuclear Physics, Novosibirsk, Russia

45: Also at Faculty of Physics, University of Belgrade, Belgrade, Serbia

46: Also at INFN Sezione di Pavia ${ }^{a}$, Università di Pavia ${ }^{b}$, Pavia, Italy

47: Also at University of Belgrade, Belgrade, Serbia

48: Also at National and Kapodistrian University of Athens, Athens, Greece

49: Also at Riga Technical University, Riga, Latvia

50: Also at Universität Zürich, Zurich, Switzerland

51: Also at Stefan Meyer Institute for Subatomic Physics (SMI), Vienna, Austria

52: Also at Adiyaman University, Adiyaman, Turkey

53: Also at Istanbul Aydin University, Istanbul, Turkey

54: Also at Mersin University, Mersin, Turkey

55: Also at Piri Reis University, Istanbul, Turkey

56: Also at Ozyegin University, Istanbul, Turkey

57: Also at Izmir Institute of Technology, Izmir, Turkey

58: Also at Marmara University, Istanbul, Turkey

59: Also at Kafkas University, Kars, Turkey

60: Also at Istanbul University, Istanbul, Turkey

61: Also at Istanbul Bilgi University, Istanbul, Turkey

62: Also at Hacettepe University, Ankara, Turkey

63: Also at Rutherford Appleton Laboratory, Didcot, UK

64: Also at School of Physics and Astronomy, University of Southampton, Southampton, UK

65: Also at Faculty of Science, Monash University, Clayton, Australia

66: Also at Bethel University, St. Paul, USA

67: Also at Karamanoğlu Mehmetbey University, Karaman, Turkey

68: Also at Purdue University, West Lafayette, USA

69: Also at Beykent University, Istanbul, Turkey

70: Also at Bingol University, Bingol, Turkey

71: Also at Sinop University, Sinop, Turkey

72: Also at Mimar Sinan University, Istanbul, Istanbul, Turkey

73: Also at Texas A\&M University at Qatar, Doha, Qatar

74: Also at Kyungpook National University, Daegu, Korea

75: Also at University of Hyderabad, Hyderabad, India 